\title{
Working towards an ideal inclusive education model for refugee background people with disabilities in New Zealand
}

\author{
Lucy Madeleine Croft
}

A thesis submitted in partial fulfilment of the requirements for the degree of Masters of Science, majoring in Geography,

School of Geography, Environment and Earth Sciences,

Victoria University of Wellington.

(2016) 



\section{Table of Contents}

Working towards an ideal inclusive education model for refugee background people with disabilities in New Zealand ................................................................ 1

Table of Contents ...................................................................................

List of Tables and Figures..................................................................... vi

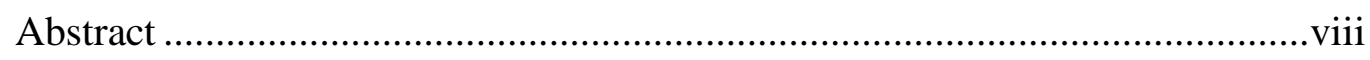

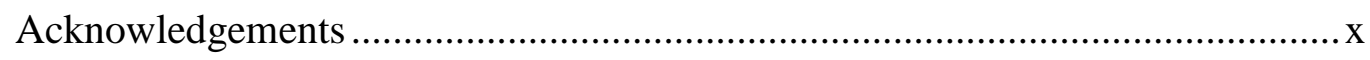

Chapter One: Introduction....................................................................... 1

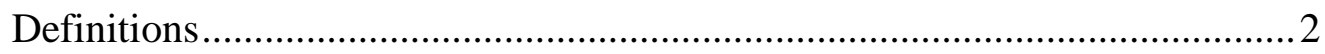

Education and refugee resettlement ................................................... 4

Inclusion, refugee backgrounds, disability and space …............................... 9

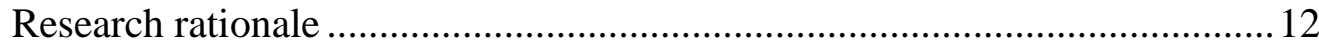

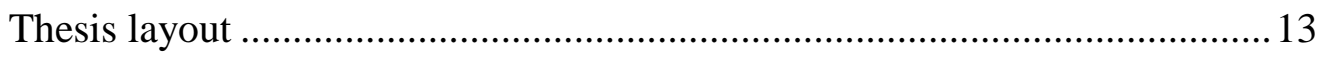

Chapter Two: Disability, Inclusion and Refugee Background People in

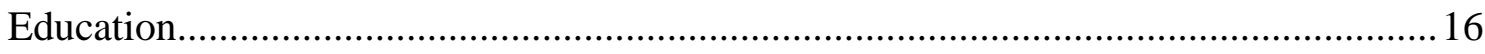

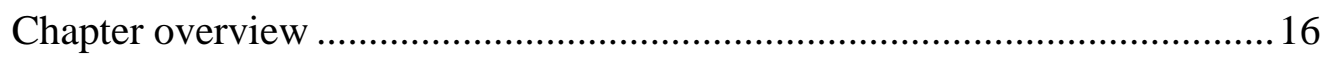

Research context: Refugee education in New Zealand and Australia ............ 17

Disability and inclusive education ...................................................... 19

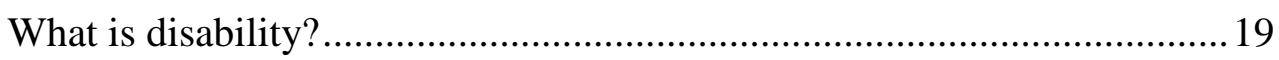

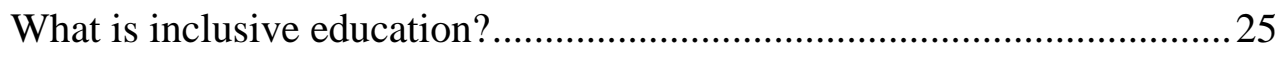

How do New Zealand schools work towards more inclusive education spaces for refugee background people with disabilities? ....................................2 27

Theoretical approach in thesis to disability, inclusion and space ............... 33

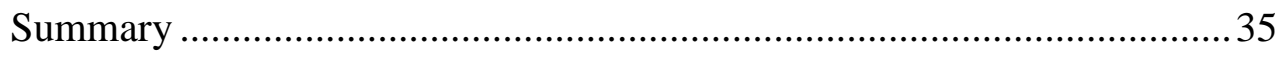

Chapter Three: Epistemology and Methodology ............................................. 37

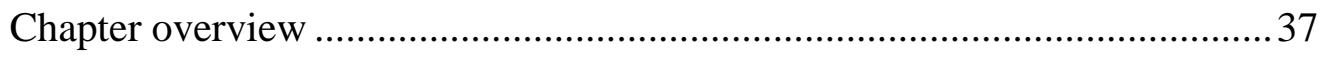

Epistemological research framework .................................................. 37 


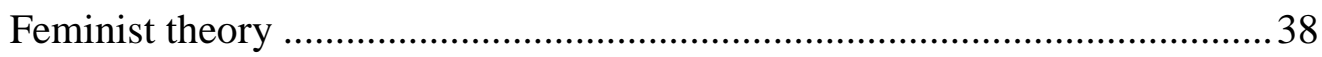

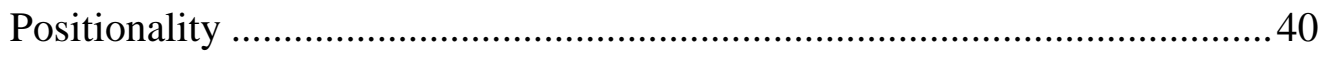

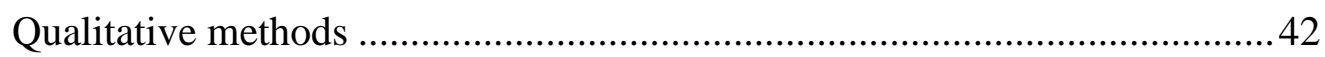

Selection and invitation of participants .................................................. 43

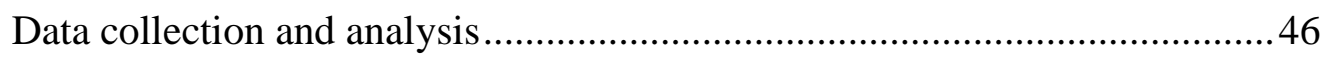

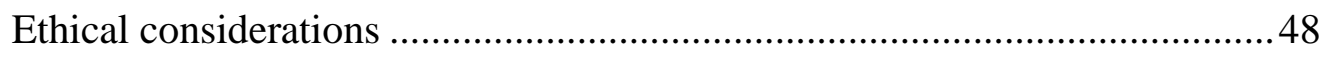

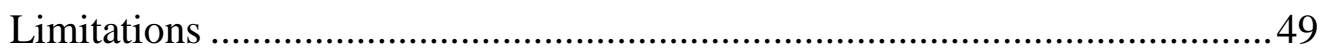

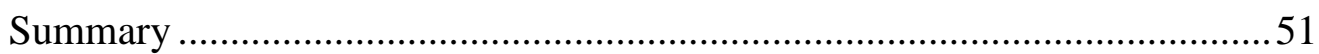

Chapter Four: Participants' perceptions of inclusion, disability and inclusive

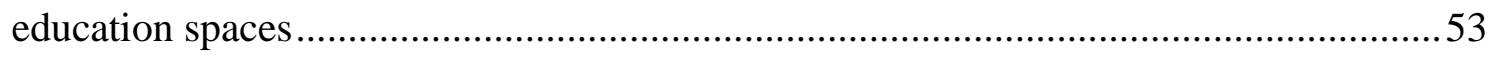

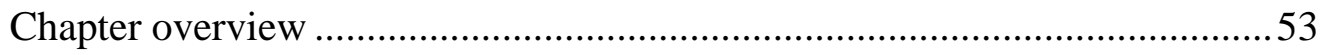

Defining inclusion and disability ....................................................... 53

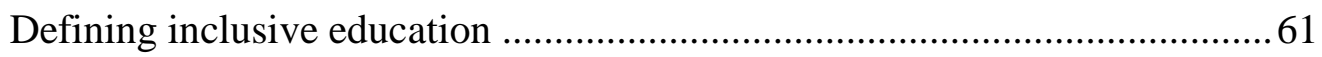

Provision of separate specialist facilities or mainstreaming in education.......66 66

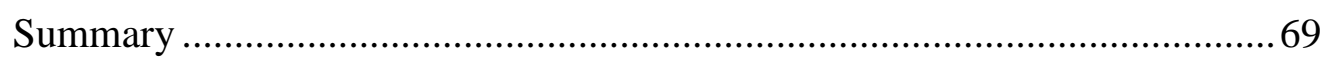

Chapter Five: Design and delivery of inclusive education spaces ................... 71

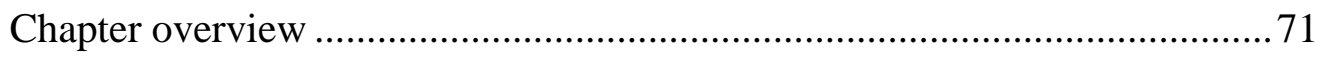

Challenges of implementing inclusive education ........................................ 72

Labelling and perceptions of disability .............................................. 72

Limited funding for refugee background people with disabilities ............. 75

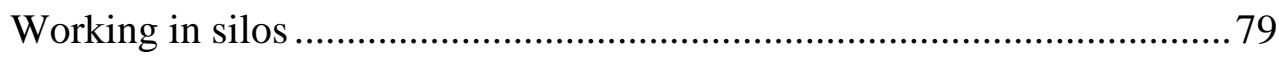

Participant perceptions on the construction of inclusive education spaces..... 80

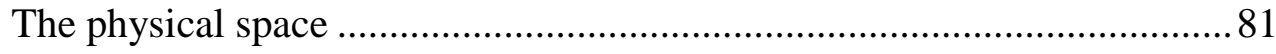

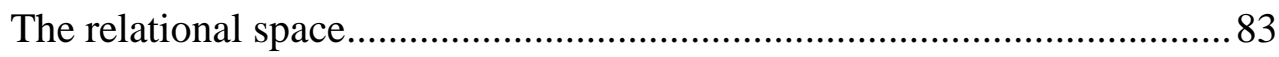

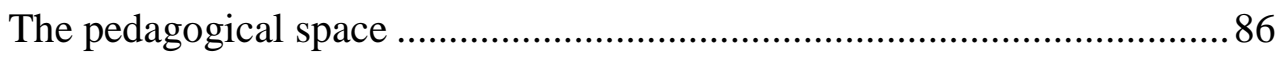

Towards a holistic, integrated model of inclusive education ........................ 91

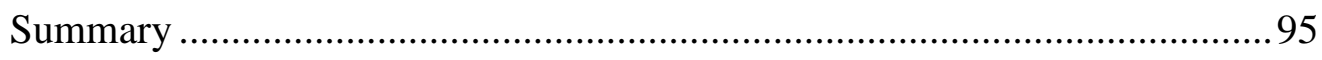


Chapter Six: Concluding remarks and recommendations for future research and

practice

Chapter overview

Overall conclusions

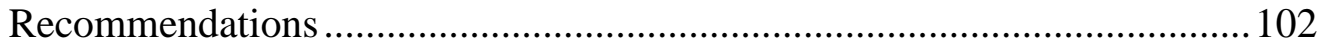

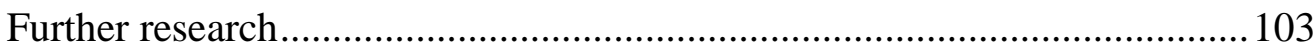

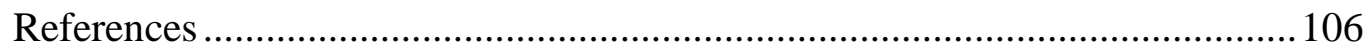

Appendix One: Interview Information Sheet ............................................... 119

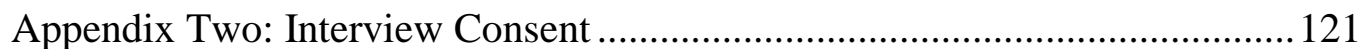

Appendix Three: Interview Questions .......................................................... 123 


\section{List of Tables and Figures}

Table 2.1: Description of models of disability .21

Table 3.1: Description of participants. .43

Figure 5.1: The three intersecting spaces required for inclusive education ...88 


\section{Abstract}

New Zealand accepts up to 750 refugees per annum, with a category for refugees with disabilities, as part of its quota obligation under the United Nations Convention relating to the Status of Refugees (1957) (Immigration New Zealand, 2016).

Immigration New Zealand's Refugee Resettlement Strategy states that education is one of the main priorities with helping refugees resettle in New Zealand (Immigration New Zealand, 2013) Although there is some literature available on refugee background people and education in New Zealand, there is little focus on refugee background people with disabilities in education. This research explores how inclusive education spaces for refugee background people with disabilities could be implemented, and perceptions surrounding disability and inclusion.

In order to gain insights into the perceptions of people involved in policy or practice with refugee background people with disabilities, I used a feminist, qualitative methodology, and conducted semi-structured interviews with 11 participants who worked in education provision, non-government or government organisations that worked with refugee background people. These participants were interviewed in Auckland $(n=4)$, Wellington $(n=3)$ and Melbourne, Australia $(n=4)$. The Australian participants were interviewed in order to provide an alternative view to their New Zealand counterparts, although the primary focus was on New Zealand.

My findings suggest that participant perceptions of disability and inclusion generally followed social and medical models of disability, but rarely ecological. Participants who have direct experience with disability of refugee background people had more carefully constructed ideas. Based on participant answers, I developed an ideal inclusive education model encompassing physical, relational and pedagogical spaces, which could be applicable to refugee background people with disabilities. The thesis findings informs existing theoretical models and understanding of inclusive education spaces, and encourages greater inclusion of refugee background people with disabilities in education in New Zealand. 


\section{Acknowledgements}

I am indebted to the people who have helped me throughout this research process, and I hope that my research leads to new insights and encouragement for others to research in this area.

Firstly, to all the participants of my study. Thank you for sharing your insights and for answering some challenging questions. The discussions we shared, and your contributions to this research are much appreciated.

To Sara Kindon and Bronwyn Wood. Thank you both for being excellent supervisors, and for all the support you provided during this thesis. It has been a privilege to work with you two, and I cannot thank you both enough.

To Disability Services at Victoria University of Wellington, particularly Rachel Anderson-Smith and Jo Hodge. Thank you for supporting me through both undergraduate and postgraduate study at Victoria, and helping me get to Melbourne to present research.

To the trustees of the Sir Roy McKenzie Scholarship. Thank you for the financial contribution to my studies at both undergraduate and postgraduate level. I wouldn't be where I am today without it.

To my friends, both near and far. Thank you all for your continued support throughout this process, and for putting up with my erratic communication when deadlines loomed! Special thanks to my fellow masters research buddies for those jugs of sangria, pots of coffee and the infamous karaoke sessions at Red Hill. Additional thanks to Erikka Helliwell and Laura Mason, two dear friends who helped with proofreading. 
To my family, in every definition of the word. Thank you all for your constant support, pick-me-up phone conversations and texts. Special thanks to my parents for fostering my love of learning, and championing my access to education from day one.

Last, but never least, to my partner, Hamish Cameron. Thank you for sharing this journey with me, for the late nights writing our theses, your proofreading, and the companionship and love you share with me every day. You're my rock. 


\section{Chapter One: Introduction}

This thesis attempts to 'fill' a current research gap by looking at inclusive education spaces for refugee background people with disabilities. Participants from a range of education providers, government and non-government organisations were interviewed to determine what inclusive education spaces already existed for refugee background people with disabilities, and how these spaces could be improved. There is limited research on how inclusion is practised, and how different models of inclusion are perceived in educational spaces for refugee background people, and their transition into a new country. In addition, refugee background people with disabilities are an often overlooked group of people within academic literature and policy decisions. I was motivated to research inclusive education spaces for refugee background people as I am passionate about ensuring equitable outcomes and access to society for all.

In this thesis I will argue that the stakeholders who inform and create inclusive education spaces need to include refugee background people, with or without a disability. I will argue that inclusive education spaces should be holistic, and consider the physical, emotional and intellectual aspects of education. My central thesis argument is that refugee background people with disabilities deserve equitable access to education. In addition, education and resettlement needs to be inclusive for refugee background people with disabilities. There needs to be consideration of the unique factors leading to a person becoming a refugee, and their potential effects upon education. This thesis focuses specifically on the perceptions and understandings of people involved in policy and provision of education for refugee background people New Zealand. However, participants from Melbourne, Australia were also interviewed in order to provide insight and comparison into the different country responses of refugee background people.

This introductory chapter provides a background to the thesis. I discuss education and refugee resettlement, and provide a broad overview of previous studies undertaken in inclusive education and geographies of disability. I outline the aim and 
objectives of this thesis in relation to the research gaps outlined. I also provide a research rationale and an overview of policy surrounding inclusive education spaces for refugee background people in New Zealand. Finally, I briefly state my positionality and methodology used in the thesis, and the thesis outline.

\section{Definitions}

This thesis contains several key terms and phrases which can have contested definitions. Definitions are always contested between people, but these definitions stand for the purpose of this thesis. In this section I will outline a number of key terms I use and describe how I am defining them for the purpose of this thesis. In general I have used 'official' definitions provided from organisations such as the United Nations, but I recognise these terms can be debated at length.

The United Nations 1951 Refugee Convention defines a refugee as:

1. “ a person who is outside his [sic] country of nationality or habitual residence

2. has a well-founded fear of persecution because of his [sic] race, religion, nationality, membership in a particular social group or political opinion, and

3. is unable or unwilling to avail himself [sic] of the protection of that country, or to return there, for fear of persecution"

(Parliamentary Library \& Parliament of Australia, 2011; United Nations High

Commission for Refugees, 1957).

A Convention refugee differs from an asylum seeker. The United Nations High Commission of Refugees (UNHCR) states that an asylum seeker is a refugee whose refugee status has not been confirmed (United Nations High Commission for Refugees, 2015). It is important to note that this thesis specifically relates to refugee background people with disabilities, not asylum seekers. Although there are similarities between refugee background people and asylum seekers, asylum seekers do not have any rights to anything in New Zealand until their applications for asylum are approved, and they become refugees. Until this happens, asylum seekers are not able to access education in New Zealand. 
In this thesis I refer to, and define, refugee background people with disabilities as refugee background students. Although the majority of literature on inclusive education and refugee background people focuses on youth, my focus for this thesis was on refugee background people who are students - many of whom could be adults as well as children and young people. Moreover, the people who I interviewed for this thesis worked with refugee background people of all ages. Additionally, people with disabilities often experience youth and the transition to adulthood at different stages, compared with the mainstream population (Hogansen, Powers, Geenen, GilKashiwabara, \& Powers, 2008; Stewart et al., 2014). Consequently, I decided to not limit my discussion of refugee background people with disabilities further by adding an age limit.

Alongside New Zealand's UNHCR ratified obligations, New Zealand has ratified the United Nations Convention on the Rights of Persons with Disabilities in 2008. The purpose of the Convention is to "promote, protect and ensure the full and equal enjoyment of all human rights and fundamental freedoms by all persons with disabilities, and to promote respect for their inherent dignity." (United Nations, 2006). Article 24 particularly recognises the right of people with disabilities to an education (United Nations, 2006). In ratifying this Convention, New Zealand (as a State Party) must ensure an inclusive education systems and lifelong learning. Under Article 22 of the 1951 Convention and 1961 Protocol, New Zealand is obliged to provide refugee background people with the same level of education afforded to national citizens (McBrien, 2014; United Nations High Commission for Refugees, 1957).

New Zealand also has a National Disability Strategy which was implemented in 2001. The Strategy aims to create a fully inclusive New Zealand society. Objective three states that the government is to ensure the best education for disabled people. Actions 3.3 and 3.4 of the Strategy are particularly relevant to inclusive education:

"3.3 Ensure that teachers and other educators understand the learning needs of disabled people.

3.4 Ensure that disabled students, families, teachers and other educators have equitable access to the resources available to meet their needs." 
(Minister for Disability Issues, 2001).

New Zealand is also a state party to the United Nation Convention Relating to the Status of Refugees (1951) and the 1961 protocol that followed. New Zealand also follows the UNHCR 'Ten or More' policy, adopted in 1973, which encourages resettlement countries to accept ten or more refugees with disabilities each year.

However, New Zealand frequently does not fill the 75 medical/disabled category places (Brandon \& Bloom, 2014). In the 2013/2014 year, twelve people were accepted under the Medical/Disability category. Only five people were accepted under this category in the 2014/2015 financial year (Immigration New Zealand, 2015). This number can be contentious, as some refugee background people with disabilities do not want to be labelled as such (Brandon and Bloom, 2014). .

Brandon and Bloom (2014) state that one of the reasons for low numbers in the Medical/Disability quota is the strict criteria imposed by the UNHCR. The UNHCR also occasionally presents cases where disability is not the primary need for resettlement (Brandon \& Bloom, 2014). In order for a person to be accepted under the UNHCR, they must have a medical condition which cannot be treated in their home country, and for which resettlement in New Zealand would significantly enhance their wellbeing. If a person has a physical or psychological condition, documentation must be provided. The applicant's family and/or dependent family members are generally included under the medical/disability category as well (New Zealand Government, 2014).

\section{Education and refugee resettlement}

New Zealand accepts 750 refugees each year as part of a quota agreed upon with the UNHCR in 1987. Each person who enters New Zealand under the refugee quota becomes a permanent resident, and is entitled to access public education (O'Connor \& Hooper, 2014). There are three subcategories within the quota for Women at Risk, UNHCR Priority Protection, and Medical/Disabled (Brandon \& Bloom, 2014). During the period July 2015 - March 2016, there were 716 refugee arrivals in New Zealand (Immigration New Zealand, 2015). Some of these arrivals were an 'extra intake' of Syrian refugees as part of the New Zealand Government's response to the Syrian crisis (Immigration New Zealand, 2016). However, only three people were accepted under the 
medical/disability category (Immigration New Zealand, 2015). Refugee background people who arrive in New Zealand under the quota spend their first six weeks at Mangere Refugee Resettlement Centre in Auckland. This programme is run by Immigration New Zealand and NGOs. This is the only such residential programme for refugees in the world and therefore is unique to New Zealand. There are around six intakes each year, with up to 125 people in each intake. The controlled intake numbers differs from other countries who are dealing with far more asylum seekers. One reason for this difference could be New Zealand's geographical isolation. In the intake programme, there is a strong focus on health and mental health, an introduction to New Zealand culture and history, employment planning, and English language education (Immigration New Zealand, 2016).

A key focus in the education of refugee background people is upon future employment (Immigration New Zealand, 2013; Marlowe, Bartley, \& Hibtit, 2014). The New Zealand government aims to improve the labour force skills of refugee background people. Immigration New Zealand and the Ministry of Business, Innovation and Employment (2012) jointly presented a co-ordinated approach to refugee resettlement services in New Zealand. These services mainly focus on providing basic housing provisions for refugee background families, and encouraging them to become part of the community by joining the workforce (Department of Labour, 2011; Immigration New Zealand, 2013). While both services are beneficial, there is little recognition of the social, cultural, individual and community health dimensions of resettlement that refugee background families experience when arriving, and settling into, a new home in New Zealand. In addition, little information is provided on the mental health and disability support available in New Zealand for refugee background people.

The New Zealand Resettlement Strategy (Immigration New Zealand, 2013), the most recent refugee strategy, is a key New Zealand government framework associated with this thesis. The overarching goal for the strategy is to ensure that refugee background people participate socially and economically in New Zealand life. The five goals of the strategy are self-sufficiency, participation, health and wellbeing, education and housing (Immigration New Zealand, 2013, p. 3). The strategy states that refugee resettlement is "a complex process that needs many different organisations to act 
together. Success in any one area relies on the success of the other areas" (Immigration New Zealand, 2013, p. 10). The strategy indicates that the New Zealand government is aware that improving educational outcomes for refugee background people will benefit other aspects of resettlement. In a presentation to participants at the National Refugee Resettlement Forum, Andrew Lockhart, National Manager (Refugee and Protection) at Immigration New Zealand mentioned the New Zealand Refugee Resettlement Strategy focused on English language provision, including "mapping alignment and provision" (Lockhart, 2015, p. 5). This included an audit of outcomes from the six weeks at Mangere Resettlement Centre, and "initial resettlement”(Lockhart, 2015, p. 5). However, there was no indication of the length of time dedicated to English language training and education after this initial period.

In recent discussions surrounding the New Zealand Refugee Resettlement Strategy, Jodi McBrien (2014) discussed the Refugee Resettlement Strategy and the different priorities held by the New Zealand government and non-government organisations. One concern highlighted by non-government organisations was the New Zealand Government's strong focus on employment and moving resettled refugees into employment as soon as possible (Altinkaya \& Omundsen, 1999; Matthews, 2008). McBrien found that the new Resettlement Strategy prioritises learning English language which focuses on employment, and places health and education as secondary goals of the strategy. Her research found that placing the secondary goals before the primary goals would allow refugee background people to develop strong skills from education which would have long term employment benefits. She also found that ensuring refugee background people were physically and mentally healthy would increase their educational and employment opportunities (McBrien, 2014). Her work highlights the need for further research in this area, and the importance of education in refugee resettlement.

The research for this thesis took place during wider intense political and media discussions surrounding refugees in New Zealand and the world. The debate in New Zealand centred on whether New Zealand is playing a strong enough role in refugee resettlement. In 2015, this fuelled a heated debate over whether New Zealand should increase the refugee quota in response to the increased number of refugees arriving from 
the Middle East, particularly Syria (Stephens, 2015). Public pressure encouraged New Zealand's current National government to allocate an extra 600 places to Syrian refugees over the next two and a half years (from 2015-mid 2017) (Immigration New Zealand, 2016). In mid-2016, the New Zealand government will decide whether to increase the refugee quota as a whole, or change the countries from which it accepts refugees (Radio New Zealand, 2015a). As a result of the increased number of refugees expected to enter the country, more regions are opening up services to refugee background people. One notable region is Dunedin, where volunteers have been training to receive the refugee background people when they arrive (Radio New Zealand, 2015b).

As a result of these events and media coverage, it is likely that refugee background people will continue to be a strong focus in the near future. There are likely to be several discussions over how New Zealand makes sure that refugee background people are welcome and provided for. The ongoing arrival of refugees and other migrants presents a number of challenges to society - in particular, the need for finance, employment, independence and integration (O'Connor \& Hooper, 2014; O’Donovan \& Sheikh, 2014).

This situation brings education into centre stage as education is one way of mitigating barriers refugee background people may face integrating into New Zealand society (Marlowe et al., 2014; McBrien, 2014). Education empowers people, helps to foster relationships, and helps people to form an understanding of their new country's culture (Taylor \& Sidhu, 2012). Prior to arriving in New Zealand, refugee background people are educationally disadvantaged, as refugee background people have broken educational histories and therefore present with some quite specific educational needs (Changemakers Refugee Forum, 2011). Taylor and Sidhu argue that this educational disadvantage, when compared to peers in Western societies, must be recognised and mitigated through inclusive education policies and practice (Taylor \& Sidhu, 2012).

It is difficult to have an educational policy which encompasses all refugee background people, as each person comes from different circumstances. In addition, not 
all educational providers have the facilities available to provide suitable educational support for refugee background people. From observing literature and policies surrounding inclusive education, it appears that most available information is for refugee background youth transitioning into New Zealand and Australian society (see Chapter Two for further discussions on these resources). There is little information available on how older refugee background people with disabilities are treated within the education system. The majority of older people rely on volunteer services to provide them with English language training, and other necessary skills. Older women are also more likely to access education and English language training. This can create disparities and inequalities within refugee background families; it becomes difficult for families to assimilate into New Zealand and Australian culture when there are family members lacking language skills (O’Connor \& Hooper, 2014). In addition, older family members rely on younger members to be translators, which requires them to come to appointments with service providers during school time. This can disrupt educational processes further, and also further alienate refugee background youth from their peers (Changemakers Refugee Forum, 2012; O’Connor \& Hooper, 2014).

It is important to recognise the effect of place and space upon the resettlement process for refugee background people, and also on educational policies. An important distinction to be made in this thesis is between formal schooling and wider education. This thesis focuses on how formal education institutions provide inclusive spaces. For many refugees, formal schooling can be disrupted, of poor quality or minimal. However, refugee background people may still be well-educated despite these schooling disruptions (O’Connor \& Hooper, 2014). Taylor and Sidhu (2012) stated that education plays a significant role in refugee resettlement, and helps to facilitate the transition to a new culture. There is a disparity between human rights agreements that democratic governments (such as New Zealand and Australia) have signed, and the refugee education policies and practices in place. Taylor and Sidhu state that despite citizenship being granted to refugees, the current inclusive education policies and practices in place contribute to the marginalisation of refugee background people (2012, p. 41).

Neoliberal governments (such as the New Zealand and Australian governments) consider exemplary citizens to be "self-sufficient, productive, responsible and 
entrepreneurial" (Taylor \& Sidhu, 2012, p. 42). In an increasingly neoliberal education environment, more holistic needs of refugee people could be neglected. Neoliberal economies can also have negative impacts upon people with disabilities. People are measured in terms of their economic worth, and their individual contribution to society. This demeans supportive, inclusive environments created for people with disabilities and refugee background people, as there is an increased focus on materialism (Nairn \& Higgins, 2011; Šiška \& Habib, 2012). An individual's ability to meet these expectations is dependent upon historical, market and societal factors. Race, class and gender all influence the state's expectation of economic performance. Taylor and Sidhu (2012) suggest that there is bias in favour of highly-educated immigrants, rather than refugees who have limited language skills. This bias can affect the provision of services for refugee background people, and particularly refugee background people with disabilities. There can also be social stigma associated with refugee background people, which affects their transition into New Zealand society through education (Calder, 2014; O’Connor \& Hooper, 2014).

\section{Inclusion, refugee backgrounds, disability and space}

The focus of this thesis is on inclusive education spaces for disabled refugee background people. These terms require further definitions at the outset. The language surrounding inclusive education changes depending on the country. This thesis defines inclusive education as including democracy, collaborative decision making, diversity, and a curriculum that is relevant to students (Curcic, Gabel, Zeitlin, Cribaro-Difatta, \& Glarner, 2011). 'Special education' is another term which is commonly used. 'Special education' emphasises that disabled people are considered to be the 'other' within education, and that their treatment should reflect their otherness (Rutherford, 2012). Conversely, 'inclusive education' suggests that people with disabilities should be equally considered in education. The term 'inclusive' suggests that including everyone will have equitable outcomes within education. 'Inclusive' also emphasises that people with disabilities may have different needs when compared to other, 'normal' people. However, these needs will enable people with disabilities to achieve the same outcomes as their peers. Therefore, 'inclusive education' denotes equity, whereas 'special education' denotes stigmatism (Collins \& Coleman, 2008; Taylor \& Sidhu, 2012). In this thesis I will use 'inclusive education' to describe educational policies which 
accommodate and cater for people from refugee backgrounds and people with disabilities.

One of the challenges of inclusive education is defining inclusion. Nguyen and Mitchell (2014) state that 'inclusion' has different meanings in Western and nonWestern societies. Western society characterises inclusion as "an ethical, political and ideological project", and identifies alternating values on difference in the education system (Nguyen \& Mitchell, 2014, p. 326). The discourse surrounding inclusion in nonWestern societies is developed through the historical effects of colonialism and exclusion. Ultimately, Nguyen and Mitchell (2014) state that inclusion is a theoretical concept which challenges institutional exclusions based on difference.

Alongside the challenge of defining inclusion is the challenge of integrating different definitions of inclusion into an educational context. Refugee background students who arrive in a Western country may not be accustomed to different education practices in Western societies. In order to formulate successful educational policies and spaces for refugee background students, there needs to be an awareness of different definitions of inclusion between cultures. Refugee background people with disabilities may not be aware that education is considered a 'right', as historically, people with disabilities have been excluded from educational opportunities in all societies. In particular, women with disabilities have been ostracised in societies over time, but have been considerably more disadvantaged in developing countries (Nguyen \& Mitchell, 2014).

The term 'refugee background', while acknowledging an aspect of an individual's history, also obscures the diverse cultures, migration histories and multiple identities of refugee background people. Refugee background students are likely to be grouped in English for Speakers of Other Languages (ESOL) classes as a collective, and little consideration is made for individual needs (Calder, 2014). In addition, it is important to recognise that refugee background people may not identify themselves with that label. Cultural or ethnic differences can also have a significant effect on 'belonging' in schools for refugee background people. Students may find the transition 
between home and school cultures difficult, which could negatively affect their educational outcomes (Calder, 2014; Kirk \& Cassity, 2007; Pinson \& Arnot, 2010).

Disability is a difficult term to objectively define, as the term is used in different contexts, spaces and places. Definitions of disability have shifted over time, and debates often occur over what is considered to be the appropriate term for people with disabilities. There are different types of disability, and a wide range of terms associated with each type. Over time, some terms have become offensive (such as using idiot or imbecile to describe an individual with an intellectual disability). It can be difficult to broadly define aspects of disability which represent everyone's views (Hall \& Kearns, 2001).

The term 'disabled people' carries political power, as it can emphasise society's oppression of people with disabilities; past and present. Geographies of disability assert that disabled people have been oppressed socially, culturally, economically, and spatially over time, which has affected their interactions in society. Historically, disabled people have created spaces at the edge of mainstream society, where they felt accepted and included. As societies move towards acceptance and inclusion of people with disabilities, the 'disabled space' and the 'mainstream space' have begun to merge; creating a wider area of inclusion (Garland-Thompson, 2004, p. 79; Gleeson, 1999, p. 48). Yet, despite this merging, it is apparent that there are still areas where disabled students receive different treatment and experience different levels of inclusion (Holt, 2003; Rutherford, 2008). Therefore the focus of this research reflects this concern, for refugee background students in particular.

The terms used in this thesis were chosen by my interpretations of disability, and what terms are currently present in literature and considered appropriate to use. Where direct quotes from participants are used or discussed, I have used the language that participants used as part of the research analysis. Within this thesis I use the phrases 'disabled people' and 'people with disabilities' interchangeably. Both phrases have politically charged meanings. 'Disabled people' suggests that disability has been excluded by society, and people's disabilities have been used as their defining aspect. In 
contrast, 'people with disabilities' suggests that disability is a characteristic which people choose to 'own', and the person is more than their disability. As disability depends on the individual, it is up to the individual to determine which 'labels' they wish to adopt (Garland-Thompson, 2004).

\section{Research rationale}

There are several reasons for the research rationale. Firstly, there is little information available on refugee background people with disabilities, as evidenced from the statistics available in Australia and New Zealand (Australian Government, 2016a; Immigration New Zealand, 2015). Unlike New Zealand, Australia does not have a specific quota for refugee background people with disabilities entering the country. In addition, the few studies that have been conducted with refugee background people with disabilities have focused on access issues in the healthcare sector (for example, access to healthcare, language barriers between healthcare professionals and refugee background people) (Changemakers Refugee Forum, 2012). Lastly, the spatial elements of inclusive education need more attention, and how space and place are important for inclusive education.

I chose to include Melbourne as a site in this research study as interviewing Australian participants was one way of evaluating New Zealand's current policies surrounding inclusive education for refugee background people with disabilities. Australia accepts more refugees each year due to its larger size, and therefore has a larger government and non-government organisation supporting them. Melbourne also has a high number of refugee background people present in the city. The majority of support for refugee background people is present in urban areas, which influenced the urban-centric approach to this thesis (Australian Government, 2016a; Changemakers Refugee Forum, 2012).

This research was also fuelled by my own passion for inclusive education, based on my own experience as a disabled woman growing up in New Zealand. I identify as a Pākeha, disabled young woman. I was born with cerebral palsy (which manifests through facial palsy) and a hearing impairment. The combination of these impairments 
means that I have experience of both invisible and visible disability. However, my experience does not account for the experiences of other people with disabilities. I was fortunate to receive an education that was mostly inclusive. However, I am aware that my educational experience would have been different if I had been brought up under different circumstances.

My positionality, and my previous educational experiences, influenced my decision to undertake this thesis. They also influenced my interactions with participants during interviews. Consequently, my research results are partially biased because of the effect my positionality had upon participant interactions. An interest in working with refugee background communities, coupled with an interest and personal drive to 'help' people with disabilities ultimately led to the decision to undertake this thesis. These motivations and review of research and literature in this field led to the research questions stated in Chapter Two.

I used a social constructivist epistemology and feminist theory to influence qualitative approaches to research. Using a social constructivist epistemology allowed me to explore socially constructed knowledge related to inclusive education and refugee background people with disabilities (Creswell, 2014). This approach influenced how I conducted interviews with eleven participants in Auckland, Wellington and Melbourne to obtain the thesis results.

\section{Thesis layout}

Chapter Two examines key aspects of existing literature which relates to inclusive education spaces for refugee background people with disabilities. Of particular note is Table 2.1, which identifies different models of disability for further discussion in the thesis. I review inclusive education policy and literature in New Zealand and Australia, inclusive education and disability, and wider theoretical approaches. I also underline the thesis research questions. 
Chapter Three outlines the research methodology. I discuss and provide reasons why my research uses a social constructivist approach and uses feminist methodologies. I outline my use of qualitative research techniques, particularly semi-structured interview questions, when interviewing participants and analysing their responses. I analysed participant responses using thematic analysis. In this chapter, I explain my motivations for research, detail my positionality and its impact upon my research outcomes and methodology. I also outline the qualitative approach and processes that I used to obtain and disseminate data. This chapter also looks at the ethical considerations and limitations to my research, and how I endeavoured to mitigate these.

Chapters Four and Five outline the empirical part of my research through an examination of my research interviews with key stakeholders in refugee education. Chapter Four discusses participants' perceptions of disability, inclusion and inclusive education spaces. These discussions are linked to models of disability, and existing literature on inclusive education. Chapter Four answers research question one in this thesis. Chapter Five continues the discussion generated in Chapter Four, by discussing the spatial, relational and emotional components of inclusive education spaces. It answers research question two of this thesis. I analyse participants' discussions of challenges faced when implementing inclusive education and what their ideal inclusive education space would look like. From these discussions I develop an inclusive education model. I analyse this model and participant discussions in relation to wider theoretical frameworks.

Chapter Six presents the final findings of this thesis. I state the key definitions of inclusive education, inclusion and disability identified by participants, and their perceptions of challenges that prevent inclusive education spaces from being developed. I use the ideal inclusive education spaces suggested by participants as a basis for my inclusive education model. This model is also influenced by spatial theorists Henri Lefebvre (1991), Doreen Massey (2005) and Claudia Thiem (2009). The application of these spatial theorists to the inclusive education model centres my thesis in geography. By using a geographical approach to inclusive education, I identify some possible ways which my inclusive education model could be applied in practice. 


\section{Chapter Two:}

\section{Disability, Inclusion and Refugee Background People in Education}

\section{Chapter overview}

This chapter critically engages with key aspects of existing literature which relate to inclusive education spaces for refugee background people with disabilities, and builds upon literature discussed in Chapter One. There are three sections to this chapter which focus on (i) refugee policy and literature in New Zealand and Australia, (ii) inclusive education and disability, and (iii) wider theoretical approaches. Chapter Two culminates in a brief discussion about how theoretical aspects of inclusion, disability and education spaces influence my approach to this research. I also identify the key research gap which this research addresses and I state my three research questions to show my research focus throughout the thesis.

The first section looks at current policies surrounding refugee background people in New Zealand, and continues the discussion started in Chapter One. It also examines the literature surrounding refugee background people in education within New Zealand. This section also sets the policy context for refugee background people in Australia, with a strong focus on the state of Victoria. Although this thesis primarily focuses on New Zealand, the Australian context demonstrates similarities and differences between the two countries in regards to the perceptions, policies and practices of people involved in refugee background people with disabilities and education.

Inclusive education and different models of disability are further examined in the second section of this chapter. I also identify key literature surrounding refugee background people and inclusive education, and examine research which has previously considered how schools, institutions and policies work towards more inclusive education spaces. The final section considers how schools can create inclusive education spaces for refugee background students, as research suggests that more 
attention needed to be given to providing constructive solutions for schools which focus on increasing participation of refugee background students in education (Cassity \& Gow, 2005; Kirk \& Cassity, 2007).

The final section of Chapter Two considers theoretical approaches to inclusion, disability, difference and inclusive education spaces. These approaches relate to the previous sections and also influence my own approach to the thesis. The theoretical approaches also relate to my research methods outlined in Chapter Three.

\section{Research context: Refugee education in New Zealand and}

\section{Australia}

Refugee background people who come in under the quota have the same rights to primary and secondary education as New Zealand permanent residents and citizens. The New Zealand government acknowledges that refugee background students have more challenges in education than other students. Consequently, the New Zealand government have refugee education coordinators and English language support as well as a guide book for all schools (Ministry of Education, 2014).

Education for students with disabilities is referred to as 'special education' on New Zealand's Ministry of Education website (Ministry of Education, 2015). There are 'Specialist Service Standards' which contribute towards the Ministry of Education's goal of 'raising achievement and reducing disparities' (Ministry of Education, 2015). However, there is no specialist service which relates directly to refugee background students with disabilities, unless the student has a physical or learning disability. Mental health is not included. This suggests that in New Zealand a focus on refugee background people with disabilities is likely to not be a strong feature of education, and that this specific social group has not received much attention in the past. However, this could be expected given the small numbers of refugee background people with disabilities accepted into New Zealand each year. 
There are some factors which are known to improve refugee background people's education and integration into New Zealand. Findings from a focus group with refugee background people undertaken by the Department of Labour (2012) highlighted the importance of learning English upon arriving in New Zealand. Participants noted that learning the language was vital to their transition into their new 'home' in New Zealand, in order to feel like they belonged (Department of Labour, 2012). Focus group findings also demonstrated issues with service provision for refugee background people. The majority of refugee background people expressed thanks for the services delivered, but noted that there were long response times which could increase levels of anxiety. In addition, a lack of English language skills prevented refugee background people from following up issues.

There was also an inconsistent application of policies within government agencies and external services (Department of Labour, 2012). While there are no direct policies for disabled refugee background people, New Zealand's Ministry of Education (2014) states that "all refugees [...] require targeted assistance in order to achieve successful resettlement outcomes both for individuals and the wider society". Refugee background students in New Zealand receive five years of ESOL language support; two years intensively, and three years of standard funding. However, the term 'student' is ambiguous, and it is unclear whether the funding could be given to all refugee background people, or just school age children (Ministry of Education, 2014).

There is a marked difference in the treatment of refugee background people in New Zealand when compared with Australia. Marlowe et al. (2014) stated that most New Zealanders viewed their country as a receptive environment for refugees. In contrast, Matthews (2008) noted that racialisation has an effect upon relations with refugee background people in Australia, perhaps spurred by the post-colonial relationships already prevalent ${ }^{1}$. She suggests that education for refugee background

\footnotetext{
${ }^{1}$ Australian resettlement programmes also take illegal migrants into account, and place them in off-shore resettlement programmes (Australian Government, 2016a, 2016b). Australia's treatment of refugee background people can vary, and Australia has been criticised recently for its treatment of asylum seekers. Although this thesis does not specifically focus on asylum seekers, there was still discussion surrounding asylum seekers when interviewing Australian participants. There was a recognition that asylum seekers also face the same barriers to inclusive education as refugee background people, but to a greater extent. Australia's asylum seeker policies have been criticised by the United Nations Human Rights Council (Refugee Council of Australia, 2015b).
} 
people in Australia was 'piecemeal' and dominated by psychological support for trauma (Matthews, 2008, p. 32). Refugee background students in education within Australia are often immersed in the classroom before their English language skills match their peers, and there is no provision for six weeks of immersion and English classes like in Mangere Resettlement Centre in New Zealand (Marlowe et al., 2014; Matthews, 2008). Matthews and others are critical of refugee background education in Australia, stating that schools needed to acknowledge the impact of racialization in Australian society in order to implement successful education programmes (Matthews, 2008; Sidhu \& Taylor, 2007; Taylor \& Sidhu, 2012).

In Australia, refugee background people on permanent humanitarian visas have a legal right to the same education as permanent residents and citizens. Refugee background people on Safe Haven Enterprise Visas (SHEVs) are only eligible for permanent residency if they are enrolled in study for 42 months, or work without receiving income support. The financial barriers to education can affect refugee background people's abilities to receive adequate education in Australia, especially if they have a disability and do not receive disability support. Refugees on permanent visas are eligible for 510 hours of free English language tuition, and are also eligible for a Skills for Education and Employment programme afterwards which supports further English language development. However, service providers have noticed that refugee background people are not always referred to these resources (Refugee Council of Australia, 2015a). The under-utilisation of these government initiatives can hinder further access to education and employment in Australia.

\section{Disability and inclusive education}

\section{What is disability?}

Defining disability is part of a wider project of critical studies in geography. Critical studies in geography seek to critique the imbalance of power relations in society and provide opportunities for marginalised voices to be heard (Chouinard, 2000; Gleeson, 1999). A focus on the geographies of disability emerged as part of an overarching shift towards more 'complex and critical geographies'. These geographies 
investigated the effect of exclusions upon different minority groups in the contexts of place and space. Geography began to consider the complexities surrounding meaning and identity, particularly in relation to the human body within place and space (Hall \& Kearns, 2001). Geography shifted 'inwards' as well as 'outwards' in thought processes; and began to consider the individual's place within space, as well as the collective. As a result, a specific body of literature dedicated to geographies of disability emerged in the 1990s (Chouinard, 1997, 2000; Gatens, 1999; Gleeson, 1999) which sought to confront oppression, and investigates the impact of exclusion upon an individual in place and space (Gleeson, 1999). The exclusion manifests in either an objective or subjective form. People may be excluded from a place due to the space that their body occupies, or may be excluded socially through differences in thought which result in stigma (Gleeson, 1999). The prevailing focus of this research has been in western communities and there is a significant absence of non-western disability communities in literature which needs to be addressed in order for empowerment to occur in place and space (Crooks \& Chouinard, 2006; Gleeson, 1999; Imrie \& Edwards, 2007).

The emergence of a geographies of disability sub-discipline coincided with broader shifts in theories that saw disability as a social construct rather than being biologically determined. These reflect broad shifts in theory from biomedical disease models to broader multi-disciplinary social models of health and health care (Kearns \& Andrews, 2010). This also related to the idea within feminist theory that gender was a social construct, while sex is biologically determined. Through disability becoming a social construct, the idea that disability could be a form of exclusion in society for individuals emerged. Similarly, the idea that disability had different meanings and experiences for individuals in space and place influenced geographic thought (Hall \& Kearns, 2001; Imrie \& Edwards, 2007).

One critique of disability studies is that it is rooted in Western theoretical concepts. Moira Gatens (1996) suggests that Western thought is dominated by dualisms. Dualisms, such as the body and mind, can limit 'othered' groups and their definitions of difference. The dominance of Western thought which is present in academic literature also demonstrates power which is authoritative (Gatens, 1996, p. 63). Aspects of refugee background peoples' cultures may be misrepresented in discussions; enforcing 
the dominance of Western thought and culture. The United Nations' definition of disability is widely regarded as a universal definition ${ }^{2}$. However, disability can be a complex construct (as evidenced by the different models of disability below), and a universal definition may ignore the different experiences of disability at regional levels (Šiška \& Habib, 2012).

Another critique of disability studies is the fact that disability is relative, which is rarely recognised. There is a hierarchy of acceptance of disability. 'Common' impairments, such as asthma and wheelchair-related impairments are considered to be the most accepted of all disability. Next in the hierarchy is sensory impairments (such as blindness or deafness). At the bottom of the hierarchy of acceptance is intellectual and cognitive disabilities (Snyer-Grant, 2001; Dear, Wilton, Gaber, \& Takahashi, 1997). Dear et al.(1997) demonstrate their prejudice against some disabilities in their article. They state that the stereotype of being "dangerous and untrustworthy" is "particularly true" for the drug addict category, which is ranked last in the hierarchy of acceptance of disability. Similarly, they state that the "unpredictability and aberrant behaviours" generally associated with mental conditions contributes to their lower ranking in the hierarchy (Dear et al., 1997, p. 466). This prejudice against certain disabled people emphasises societal stigmas against people with disabilities. Even within a hierarchy, disabled people are still viewed as the 'other' (Crooks, Chouinard, \& Wilton, 2008; Crooks \& Chouinard, 2006; Grech \& Soldatic, 2015). Bodies can act as boundaries which distinguish the disabled from the non-disabled. The visual representation of difference can create boundaries between people's perspectives. It can also create boundaries in the definitions given to disabilities. The need to differentiate between abled and disabled, and to label the differences, may be ingrained in the human psyche. This links to the sociological concept of labelling theory (Söder, 2006). Even when an individual is perceived to be disabled, they could still be drawing distinctions between themselves and other disabled individuals (Dear et al., 1997).

\footnotetext{
${ }^{2}$ The United Nations Convention on the Rights of Persons with Disabilities states that people with disabilities "includes those who have long-term physical, mental, intellectual or sensory impairments which in interaction with various barriers may hinder their full and effective participation in society on equal basis with others" (United Nations, 2006).
} 
These shifts in political discourses within geographies of disability and society in general have influenced the shift in terminologies and understandings over time. These shifts also corresponded with political changes and theoretical shifts in gender and sexuality studies (Gleeson, 1999). Broadly speaking, there are four key models associated with disability and education: the charitable model, the medical model, the social model and the ecological model. These models are briefly described in the table below.

Table 2.1: Description of models of disability

\begin{tabular}{|l|l|}
\hline CHARITY & $\begin{array}{l}\text { Oldest known model of disability. Suggests that the disabled person } \\
\text { is to be pitied, and is unlike other people. Disabled people should be } \\
\text { seen but not heard (Gleeson, 1999; Thomas, 2004). }\end{array}$ \\
\hline MEDICAL & $\begin{array}{l}\text { Disability is caused by, and is the responsibility of, the individual. } \\
\text { The individual's disability is seen as something which should be } \\
\text { fixed or cured. The disabled person is often othered, and considered } \\
\text { abnormal (Šiška \& Habib, 2012; Swan, 2002). }\end{array}$ \\
\hline SOCIAL & $\begin{array}{l}\text { Disability is caused by society's failure to be inclusive for everyone. } \\
\text { Society causes disability, not the individual. Society should become } \\
\text { more inclusive for people with disabilities (Gleeson, 1999; Siška \& } \\
\text { Habib, 2012). }\end{array}$ \\
\hline ECOLOGICAL & $\begin{array}{l}\text { Educational model of disability. Could be interpreted as a } \\
\text { combination of the medical and social models of disability. } \\
\text { Disability is a mixture of the individual, society and the } \\
\text { environment. Acknowledges technology's application to current } \\
\text { society creating new methods of inclusion for people with } \\
\text { disabilities (Ebersold \& Evans, 2003; Helliwell, 2014). }\end{array}$ \\
\hline
\end{tabular}

The charity model of disability suggests that the disabled person is the 'other', and should be pitied and considered as a charity case. The charity model aligned with asylums and institutions used to house people with disabilities in the 1900s, and in some cases, today. This antiquated model assumes that disabled people are less intelligent and beneath able-bodied people (Gleeson, 1999; Thomas, 2004). 
The medical model of disability suggests that disability is treatable, and embraces the concept of 'mind over matter'. The individual is the source of their own impairments, and is solely responsible for managing their disability (Gleeson, 1999). Within geographies of disability, and the history of disability, there has been exclusion of certain disabilities. Currently, one invisible population within geographies of disability is intellectually disabled people (Hall \& Kearns, 2001). Historically, people with intellectual disabilities were incarcerated in asylums, which meant that they weren't 'seen' in society. Their oppression and invisibility inferred that intellectually disabled people had no place in society. Consequently, the geographies of intellectually disabled people only recently emerged as asylums and institutions are shut down (Hall \& Kearns, 2001, p. 238).

Disabled people within the medical model are often positioned alongside the concept of 'normalcy' in society. People with disabilities are often seen as the 'opposite' to 'normal' people; they are the unknown or the abnormal. This perception can be exclusionary, as normalcy does not suggest alternative options to work with the needs of the disabled. Within education, the word 'special' is often attached to refer to people with disabilities (Michailakis \& Reich, 2009). The idea of special education can be linked to the medical model of disability, as the student is required to have a diagnosis and assessment of needs in order to participate (Gable, 2014; Šiška \& Habib, 2012). The growing realisation of medical related disability in society has increased the attention given to those with different needs and ideas to 'normal' society. However, some disabilities remain inconspicuous, which can prevent public knowledge and awareness of difference (Hall \& Kearns, 2001, p. 240). In order for societal perceptions to shift, disability and accessibility needs must be visible. The difficulty lies in ensuring that all needs are in the foreground, when there are so many disabilities (and individual responses to disability) to contend with (Gleeson, 1999; Imrie \& Edwards, 2007).

The social model of disability asserts that disability is subjective; an aspect of one's social identity (Gleeson, 1999). The social model suggests that disability is created through society's inability to cater for everyone. Exclusion from society is the fault of the collective, not the individual. The social model states that impairment is 
diagnostic, and disability is socially constructed (United Nations, 2006). Society must be inclusive towards all people, both in mind-set and through physical constructions. However, disability is found in different types of societies, so it is difficult to have a 'one size fits all' inclusion policy for all societies (Gleeson, 1999; Thomas, 2004). The social model is the prevailing model used in societal discourses today and suggests that a more nuanced definition of disability is needed which takes into account, society in question, how people define themselves and how they wish to be treated. The social model identifies that disability is used as a comparative measure; a movement away from viewing the individual as disabled, and instead considering the space between the disabled individual and the abled-bodied ideal. If disability is considered as a definition of a person's comparative difference from the normative body, then it becomes difficult to contest what constitutes disability (Dear et al., 1997; Hansen \& Philo, 2007).

The ecological model of disability is the most recent educational model of disability. This model combines and extends aspects of the medical and social models of disability (Ebersold and Evans, 2003). It suggests a more holistic and connected notion of inclusion. It integrates developed understanding of disability in relation to space and environment, alongside the social and medical contexts. This is important as the model considers physical as well as attitudinal barriers. The ecological model recognises that a person's impairment and background can affect their experience of disability. It also recognises that the built environment affects perceptions of disability. The ecological model also includes technology in its definition of the built environment; recognising the changing role that technology plays in enabling (and disabling) individuals (Ebersold \& Evans, 2003; Helliwell, 2014). The ecological model also considers the effect of spaces on people with disabilities. Consequently, the model can be applied to people with disabilities within an education space. The model could be used as a reflexive tool to evaluate whether an educational space was inclusive and beneficial for people with disabilities, and whether the space supported people with disabilities.

The underlying evaluation of inclusive space in this thesis applies an ecological model. This approach incorporates the geographical concepts of space and place, and also considers individual and societal factors which could benefit or inhibit inclusive 
education. Mitchell (2012) states that an ecological approach is ideal for creating an inclusive education setting to working with children with disabilities. All students benefit from a common set of strategies in education, even if these strategies need to be adapted for refugee background students with disabilities. Mitchell's (2012) findings suggest that discussions of a student with disabilities' needs between the teachers, students and their families can aid the development of inclusive education spaces.

Another aspect of ecological understanding within this thesis is my positionality

and its role within my approach. Previous studies encourage more research on disability by disabled researchers. Disabled researchers, like myself, have different sensibilities and can provide additional insights from lived experiences. Doing so enriches the knowledge pool surrounding disability. I also have direct experience and understanding of the ecological model of disability, and how it can be applied to analysis (Chouinard, 1997, 2000; Crooks et al., 2008).

\section{What is inclusive education?}

Although there are several definitions of inclusive education available (see Slee( [2001, 2006), Gable (2014), Kearney and Kane (2006)), in this thesis I use Curcic et al's definition. They state that inclusive education and schools are

"those that adhere to democratic principles, including collaborative decisionmaking and practice, celebration of diversity (including ability diversity), engagement with the broader community, a curriculum relevant to students' lives and flexible learning contexts"

(Curcic et al., 2011, p. 119). Curcic et al., (2011) state that a community involves people living a life with commonalities in a variety of spaces, within a certain timeframe. The spatial and temporal elements of communities create social, political and economic systems where people within the community are placed in relation to one another, ideally within reciprocal relationships (Curcic et al., 2011).

The Ministry of Education defines inclusive education as "where all children and young people are engaged and achieve through being present, participating, 
learning and belonging" (Ministry of Education, 2016). This definition of inclusive education is contestable, as it does not directly include students with disabilities. Inclusive education principles are bonded by the Education Act 1989, which stipulates that "people who have special education needs (whether because of disability or otherwise) have the same rights to enroll and receive education at state schools as people who do not" (New Zealand Government, 1989). This definition is also interesting as it suggests that exclusion can still occur in private education. In New Zealand, disabled children tend to be 'mainstreamed' into New Zealand schools with a teacher aide, or enrolled in special schools in accordance with their disability (Macartney and Morton, 2013; Rutherford, 2012). Disabled students in mainstream education still experience discrimination by virtue of having a teacher aide present (Rutherford, 2012). In contrast, inclusive education seeks to remove all exclusionary barriers, which could, by definition, include a teacher aide.

Overall, inclusive education is a highly contested and debated term. The development of inclusive education largely follows the widening definitions of disability. These definitions range from narrow approaches with a strong relationship to the medical model of disability, to much more nuanced approaches which are linked to the social and ecological models (Kearney, 2016; Kearney \& Kane, 2006; Šiška \& Habib, 2012; Slee, 2012). The broader definitions of inclusive education focus on wellbeing in relation to inclusion.

Inclusive education was preceded by special education approaches (Ypinazar \& Pagliano, 2004). Hassanein (2015) suggests that one rationale behind inclusive education is the "psychological-educational rationale," which considers how children with "special education needs" learn, so as to identify a more inclusive education environment for them (Hassanein, 2015, p. 39). There are two dominant interpretations of inclusive education. One idea is that special education practices should merge with 'normal' education. The alternative view suggests that inclusive education should have no relationship with special education, and should instead be a new education system which caters to everyone's needs, regardless of needs and differences (Corbett, 1999; Higgins, MacArthur, \& Morton, 2013; Kearney, 2016; Kearney \& Kane, 2006; Slee, 2001; Slee, 2012). 
Creating schools which cater to all students' needs, should be favoured instead of isolating students with disabilities in segregated classrooms. In addition, there is a strong belief that students with special education needs should not have to travel in order to receive an adequate education (Kearney \& Kane, 2006). One aspect of inclusive education is the acknowledgement that a school exists beyond its physical boundaries (Kearney \& Kane, 2006). Schools can also shape how minority groups are received in communities, as inclusive education can create diverse spaces (Hassanein, 2015). However, one of the challenges that schools face is providing each student with equal opportunities once they leave the school boundaries (Collins \& Coleman, 2008).

Ensuring that refugee background people with disabilities have the choice to participate in all aspects of their schooling is an example of social and political elements of community informing a school system to make it more inclusive. Creating a space which ensures that a marginalised community can participate in education is a political act, as it counteracts the 'norm' of excluding difference (Curcic et al., 2011). Consequently, inclusive education spaces can be powerful contradictions to pervasive social and political aspects of society (Curcic et al., 2011; Singal, 2008).

\section{How do New Zealand schools work towards more inclusive education spaces for refugee background people with disabilities?}

The arrival of new students from other places can create challenges and opportunities for schools. Students are increasingly mobile - this includes fee-paying international students in both New Zealand and Australia, but also the much smaller group of refugee students which arrive every year into schools. 'New' cultures can be created from student movements, and more attention has been given to ESOL courses as more international students move to 'Western' universities (Holton \& Riley, 2013) and there are more diverse groups in New Zealand classrooms (Marlowe et al., 2014). However, an increase in ESOL provision does not automatically increase subsequent opportunities for refugee background people. Refugee background people are more likely to be excluded, especially at tertiary level as they do not pay international fees like international students and there is concern that increasingly neoliberal universities 
have created more inequalities for minorities (Holton \& Riley, 2013; Waters, 2012). In addition, refugee background students can also have specific needs which requires extra attention in educational settings.

Refugee background students often have interrupted schooling as a result of moving around or away from conflict situations. One of the major challenges for new refugee background students is finding a sense of belonging within a community. Schools are particularly important in helping to assimilate refugee background students into their new country, as well as providing education (Cassity \& Gow, 2005; Kirk \& Cassity, 2007). Furthermore, when refugee background students are disabled, they potentially experience greater levels of stigma and discrimination and disadvantage in their education (Hassanein, 2015).

The creation of separate facilities for disabled people is an aspect of the built environment which reflects attitudes towards disability. While debates in this area are heated, research shows a mixed picture of how they are experienced by people with disabilities. Segregated schools can be perceived as positive education environments for students' safety. Worth (2013) found that visually impaired young people's school lives were a key space for students to come to terms with disability and the surrounding concepts. This space was enabled through interactions with peers and teachers. Even when people with disabilities were in segregated schools, they discussed social interaction with wider school environment, demonstrating a link between positive social relationships and the education space in which these interactions occurred. However, Worth (2013) found that some visually impaired students saw specific spaces marked for students with disabilities as exclusive. Although the student in the study appreciated the help that she received from teachers in the inclusive space, her involvement with the room marked her as 'different', and lead to social exclusion by her peers (Worth, 2013).

Schools which emphasised inclusive education appeared to work beyond legal obligations required of schools. In doing so, these schools exerted a political action rebelling against neoliberalism which generally does not acknowledge ethics of care (Pinson \& Arnot, 2010; Selvaraj, 2015). While students can feel 'safe' within some 
education spaces, the act of entering these spaces serves as a form of disclosure which could negatively affect social interactions. Therefore, care needs to be used when discussing 'safety', as separate inclusive education spaces may not encourage safe spaces in all areas of education. Safety was a key word also developed in previous studies surrounding refugee background students and inclusive education (Humpage, 2009; Kirk \& Cassity, 2007; Pinson \& Arnot, 2010). The word 'safe' was often used to enhance the idea of a welcoming environment where students felt like they 'belonged' in a classroom.

Another aspect of inclusive educational space is places where people feel they belong. Understanding disability and inclusion as a relational concept can improve student experiences of inclusion. Historically, place and space have been passive components of education theory. Kenway and Youdell (2011) emphasise that emotion is not a formal aspect of education. Education is generally perceived as a rational endeavour, made up of a series of well-reasoned, logical practices. Education is therefore seen as an objective space, and emotion (as the subjective space) is not considered in conjunction with the objective. However, including place and space when considering aspects of education helps when considering student diversity and how to create an inclusive environment. Creating an inclusive education space can also help mitigate educational inequalities which may be present (Kenway \& Youdell, 2011).

Inclusive education spaces can be created through positive interactions between students, teachers, and peers. Prioritising the wellbeing of the disabled student through social interaction and 'small' changes can create a positive emotional space for the education environment (Evans, Harvey, Buckley, \& Yan, 2009). Positive social relationships boost students' educational abilities and feelings of inclusion, as Worth (2013) found in a United Kingdom study and Zembylas (2011) in his study of a multicultural primary school in Cyprus. Schools generally employ teacher aides to help provide the appropriate education support needs for students with disabilities. However, the educational support that teacher aides provide can contradict social inclusion for students with disabilities, as teacher aides can limit students' social lives with peers (Worth, 2013). Worth (2013) found that while teacher aides could benefit a student 
academically, they could also 'police' student behaviour and disallow them the opportunity to have fun with their peers.

Furthermore, the spaces designed for students with disabilities in mainstream schools can sometimes emphasise exclusion. Students with disabilities may be deliberately seated at the front of the classroom, near the teacher, or seated next to their teacher aide. The student's inability to dictate what space is preferable to them singles them out from their peers, and can negate inclusive education outcomes (Higgins et al., 2013; Holt, 2003; Worth, 2013).

A third aspect of creating inclusive spaces is the use of specific pedagogies and practices to create inclusive education spaces. Creating a space which feels welcome includes attention to affect and emotion. Affect is understood as "the intense sensation of bodies that are pre-personal and pre-discursive" (Kenway \& Youdell, 2011, p. 113). Affect is a sensation of the body, whereas emotion is how this sensation is expressed through language. The sense of belonging, attachment and familiarity become very important components of a schooling experiences for refugee background people (Marlowe et al., 2014; Taylor \& Sidhu, 2012). Marlowe et al (2014) emphasise the importance of teachers being engaged with refugee background students and their families in order to encourage belonging in education. Schools with a strong focus on social justice were also perceived as beneficial for inclusive education spaces (Landorf \& Nevin, 2007; Taylor \& Sidhu, 2012; Zembylas, 2013). The majority of studies of refugee background students in inclusive education focus on ESOL classes, and the effect of psychological trauma on education (Marlowe et al., 2014; Slewa-younan et al., 2015; Taylor \& Sidhu, 2012). There is little available on how different spaces can influence pedagogies and practices.

One way which spaces can be developed is through critical emotional reflexivity (Zembylas, 2013) as part of a range of teaching practices. Emotional reflexivity acknowledges the importance of emotions in everyday practice. Emotions can influence or interrupt everyday practice, or both. In addition, everyday practices and actions can create emotional bonds between individuals which are necessary for social change to 
occur (Zembylas, 2013). Critical emotional reflexivity was also important when developing spaces "for teachers and students to navigate the emotions of implicit activisms at the school level" (Zembylas, 2013, p. 90). By reflecting on their everyday practices and actions, both teachers and students could engage in socially just conversations and behaviours which support marginalised people and groups. Therefore, critical emotional reflexivity could be used as a pedagogical tool by teachers in order to evaluate inclusive education spaces for refugee background people. Using critical emotional reflexivity could help teachers and students to become more aware of refugee background peoples' needs in an educational space (Zembylas, 2013). Zembylas (2013) also introduced the idea of implicit activism in his discussion of critical emotional reflexivity practices in a school in Cyprus. An example of implicit activism is demonstrating care for refugee background people who may be discriminated in everyday situations. Care can be demonstrated through 'small' gestures, such as encouraging words and including refugee background people in classroom activities (Zembylas, 2013). Teachers being aware of students' needs is one example of practising implicit activism through inclusion.

Putting the above ideas together forms a way to develop a holistic education approach. This approach would incorporate elements of the built environment, sense of belonging and inclusive pedagogies discussed above. This approach fosters the students' physical and emotional wellbeing, in conjunction with the traditional academic aspects of education (Pinson \& Arnot, 2010). In Pinson and Arnot's (2010) study, they found that schools in the United Kingdom which adopted a holistic approach to education were also able to oppose anti-refugee sentiments and neoliberal discourses within education. These schools also recognised that refugee background students had different needs to the general population. Several different types of support were also set in place to cater to separate needs. Schools with holistic approaches to education also communicated with different agencies in order to meet support needs of refugee background students (Pinson \& Arnot, 2010). The interrelationships developed between schools and agencies also linked to a 'whole school' approach to refugee resettlement and education. Having a whole school and holistic approach to education developed resilience and community with refugee background students (Matthews, 2008; West, 2004). Similar to Zembylas' (2013) study, the schools also valued cultural diversity, 
relationships and models of care for students in order to foster inclusive education spaces.

Worth (2013) also emphasised the importance of relationships at school, as they teach students what to expect of societal interactions outside of the educational environment. Students using an inclusive education space for support suggests that school has an informal social component associated with the formal education component. In schools where students with disabilities are segregated, social interaction is often the chance for students to be included with their mainstreamed peers (Holt, 2003; Worth, 2013). Having a space that caters to students from particular cultures can help to facilitate inclusion through social interaction. However, there is little knowledge about how such education spaces operate for refugee background students with disabilities, or how people who work closely with refugee background people perceive inclusive space.

As a result of this literature review, part of my attempt to fill the research gap outlined in the previous section involves research questions. These three research questions are:

1) What are the definitions and interpretations of inclusion, disability and inclusive education held by key stakeholders in refugee support, education and policy?

2) What are their perceptions of the challenges of implementing inclusive education spaces for refugee background people with disabilities, and how do they perceive ideal inclusive education spaces?

3) How could participants' ideal inclusive education spaces help create a more holistic model of inclusive education for refugee background people with disabilities? 


\section{Theoretical approach in thesis to disability, inclusion and space}

In order to understand inclusive educational spaces, space needs to be considered and understood, and how therefore space could be conceived of as more or less inclusive. My approach in this thesis is informed by a number of spatial and relational theories, and particularly by Doreen Massey, Claudia Thiem and Henri Lefebvre who have theorised in this area. In the section below, I examine how theories of space and relationships can contribute to a deeper understanding of inclusive education for refugee background people with disabilities.

Space has often been considered as static, immovable and like a container. However, recently people have begun to understand space as a product of social interactions, relationships and practices (Lefebvre, 1991; Massey, 2005; Thiem, 2009). Doreen Massey (2005) also discussed the idea that space can be socially created. She states that space is more than a surface layer where temporal processes occur; it is moulded and developed by the social relationships and processes which also take place in an area. Space is constantly being made, as social processes and practices are constantly changing. Space is relational, and can never be viewed as a silo, as there are always social links and connections with other spaces. Massey's (2005) ideas can be connected to inclusive education spaces as these spaces are intimately connected with, and shaped by, the social processes that take place (Cook \& Hemming, 2011).

Lefebvre (1991) also states that spaces occupied by social practice, physicality and sensory phenomena should be considered together, rather than separately. Lefebvre described space as perceived, conceived, lived or practised (Lefebvre, 1991) and that these three elements together interact to produce space. These terms are also described as spatial practice, the representation of space and spaces of representation (Stanek, 2011). He argues that these three spaces exist in relation to one another, and affect individual interpretations of a place. Power is also a key consideration, as social spaces may be dominated by certain groups (Cook \& Hemming, 2011; Lefebvre, 1991). Social spaces can influence physical and cultural spaces, which can translate to the dominance of one culture across all aspects of space. Lefebvre's (1991) theory can be applied to the formation of inclusive education spaces and a consideration of the different spaces which make a whole. In order to examine inclusive education spaces, the physical, 
social and mental dimensions of space within education could be included to understand social processes in education (Cook \& Hemming, 2011).

Applying these ideas to the context of education means that there needs to be consideration of how educational spaces are a product of social forces and relationships. Thiem's (2009) primary argument is that education spaces both influence and are influenced by wider societal processes (Cook \& Hemming, 2011). This argument relates to earlier geographies of education research which assert that wider societal discourses influence education systems and institutions. Similarly, schools and education spaces can contribute to society through pedagogies (Collins \& Coleman, 2008; Holt, 2003). Thiem argues that while the majority of geographies of education research has been inward-focused (looking at changes within the education sector), it needs to look outwardly in order to understand how education affects change in society. She suggests that considering how education affects wider societal processes could lead to discussions relating education to wider social and political geographies (e.g. geographies of disability) (Thiem, 2009). One such way is by looking at how an individual's background influences their education, similar to my approach of creating inclusive education spaces for refugee background people with disabilities. The intersection of objective and subjective interpretations of space determine how disability is perceived by societies (Gleeson, 1999, p. 47). Both physical and mental constructions are used when imagining spaces for disability to exist in society. Disabled people occupy spaces where there has been oppression, and endeavour to reclaim these spaces so that they're inclusive. Social spaces are formed by the people who occupy them, and people's production within a space also determines how it is perceived. All spaces do not exist in a vacuum; they are all interconnected through human interactions (Gleeson, 1999; Imrie \& Edwards, 2007).

These ideas strongly influence my approach to disability, refugee background people and inclusive space. Space is a theoretical and social component of society, and can be physically or mentally constructed by individuals and/or a collective. Within geographies of disability, space can be subjective, as an individual can determine how included and/or excluded they feel in regards to their surroundings. Space can also be objective, as an individual may be excluded due to the physical surroundings, or by 
dialogue stated by peers. Space is a key aspect of inclusion, especially in the context of disability and difference as people with disabilities are often defined by the spaces that their bodies are included or excluded from (Swan, 2002). People's interpretation of space can help to determine spaces which are inclusive or exclusive. Additionally, people with disabilities and refugee background people can have different interpretations of space owing to their backgrounds, which could affect their definitions of inclusion. My thesis contributes to the wider discussion of inclusive education for refugee background people with disabilities by incorporating these geographical notions of space and place.

Policies and practices play a role in the extent to which education spaces are inclusive (Nairn \& Higgins, 2011). Therefore, in this thesis I have chosen to interview eleven participants who work in either policy or practice with refugee background people with disabilities (see Chapter Three for further detail on this). The three research questions of this thesis aim to discover practitioners' experiences with refugee background people with disabilities, and how this affects their perception of inclusive education spaces. An understanding of disability and inclusion is important when developing inclusive education spaces for refugee background people with disabilities as it will help to define what makes a space inclusive or exclusive.

\section{Summary}

Overall this chapter provides an overview to the reasons behind my research. The current literature demonstrates what studies have already been done on inclusion, disability and inclusive education for refugee background people with disabilities. The contested nature of disability and inclusive education demonstrates why I need to ask for definitions, in order to clarify stakeholder understandings. The literature also highlights that geography, particularly space, has not been a strong focus in previous studies on refugee background people and people with disabilities. Consequently, asking how space can be included in a holistic model of inclusive education will demonstrate geography's relationship with inclusive education. My research will aid current discourses in inclusive education as it brings a geographical approach to inclusive education. This will add to current literature which predominantly focuses on the psychological effects of inclusive education. The theoretical spatial approaches to 
my thesis bridge the gap between my literature review and a wider discussion of epistemology and theory in my methodology chapter, Chapter Three. 


\section{Chapter Three: Epistemology and Methodology}

\section{Chapter overview}

This chapter describes the epistemological framework developed for this project and the methodologies that I applied when exploring the provision of inclusive education spaces for refugee background people with disabilities. I used a qualitative approach to my research as my research questions required descriptive and reflectively analytical answers from participants. In this chapter I outline and explain the social constructivist epistemological position I applied and the use of feminist theory in order to investigate inclusive education spaces for refugee background people with disabilities. This chapter also outlines my positionality and its potential effects upon my research.

In this chapter I also provide a broad overview of the processes that I used to select and invite participants that I interviewed in Auckland and Wellington, New Zealand, and Melbourne, Australia. I describe the data collection methods used with participants, and how the data were analysed. I also outline the ethical considerations associated with my research, and ways that ethical concerns were mitigated. This chapter concludes with some of the limitations of my study.

\section{Epistemological research framework}

A social constructivist framework was chosen for my research as it explores the socially constructed knowledge surrounding inclusive education spaces for refugee background people with disabilities. The framework also connects with elements of qualitative research that I used throughout this research (Creswell, 2014). The social constructivist movement arose from a rejection of scientific methods and rationale and sought instead to investigate "causal processes of belief formation and information" (Detel, 2015, p. 229). Qualitative approaches can support social constructivism as these methods help to analyse social constructs from the perspective of individuals and groups involved in a variety of contexts (Creswell, 2014; Detel, 2015). 
Social constructivism rests upon an understanding that individual experiences are subjective, and cannot be objectively defined. These subjectivities can be complex, and require the researcher to holistically look at the different views that participants express. Social constructivism also acknowledges the significance of context in research, and the effect that context can have when analysing research results. No result is entirely objective (Detel, 2015). Similarly, another important aspect of social constructivism is that knowledge is situated and therefore dependent upon the context in which the knowledge is received (Thompson, 2015). These ideas also are supported by feminist theory as they both focus on the 'ontology of being' (Locher \& Prügl, 2001, p. 112). Both feminist theory and social constructivism state that knowledge can be transformative (Locher \& Prügl, 2001; Thompson, 2015). In particular, feminist geographies also have a commitment to situated knowledge, reinforcing the importance of research context (Dias \& Blecha, 2007).

Applying a social constructivist stance in this research meant that the questions that I asked participants were broad, which allowed different discussions and meanings to arise. I also sought to investigate how participants' interactions with their environment, in this case, their experience in working with refugee background children and youth in policy of education, shaped their perspectives. This is a key aspect of social constructivism (Creswell, 2014). By using a social constructivist framework, I also acknowledge that my personal background shaped my data interpretation. Therefore, my data interpretation is inherently biased based on my experiences. Overall, this framework was the basis of determining meaning that participants had surrounding inclusive education spaces for refugee background people with disabilities.

\section{Feminist theory}

Feminist theory challenges pre-conceived narratives. It questions the basis of knowledge and power within a patriarchal society (Gatens, 1999). When feminist theory can be applied to research, it encourages researchers to be reflexive and critical of power structures and oppression throughout the research process (Sultana, 2007). As my research is based upon disabled people's experiences in society, feminist theory can provide insights into experiences of inclusions and exclusions within education.

Feminist methodologies involve evaluating power dynamics and recognising that there 
are existing power structures which permeate research methodologies. My research investigated the positive and negative power dynamics which could exist within education spaces. Positive power dynamics were associated with inclusivity, and negative power dynamics with exclusion. There are different forms of power which are demonstrated in knowledge creation (Orme, 1997). Feminist research also questions the power balance which can exist between the researcher and the participant, and the consequent effects on research results (Orme, 1997). The intersection between feminist theory and the practical applications of my research (praxis) is evident throughout my methodology. I endeavoured to reduce inequalities that may have been present between the participants and myself. I also aimed to ensure that participants' perspectives informed the results, despite the interviews being conducted with pre-determined questions (Catlett \& Beck, 2007). An important aspect of applying feminist theory to my methodology is acknowledging that I may be unconsciously privileging certain aspects of knowledge over others. In order to mitigate this I used qualitative approaches and relied on semi-structured interviews to incorporate participant voices and ensure that my questions weren't always the dominant focus.

Orme (1997) stated that feminist discussions of knowledge construction were necessary in developing social research. In addition, the intersection between theory and practice (praxis) is important in developing discussions of knowledge and power in social research. Praxis is defined by Freire as "the action and reflection of men and women upon their world in order to transform it" (1993, p. 60). Freire also states that education is an ongoing activity which is remade through praxis, which is important when analysing aspects of inclusive education (Freire, 1993). Praxis enables practical applications of social research, which can be seen through problem-posing education that allows humans to move forward within education (Freire, 1993). Praxis also considers that there are different ways of existing, and there are many factors which lead to oppression in society. These aspects of praxis were important when researching inclusive education spaces, as interviewing people from different areas of inclusive education and who also work with refugee background people meant that there were different perspectives to consider. Although this thesis did not focus on participatory action research, the idea of praxis underpinned my ethical commitment (Cahill, 2007) to investigating and developing more inclusive education spaces for refugee background people with disabilities. The research was personally, politically, and professionally 
driven to contribute to social change for refugee background people with disabilities in education. Questioning participant values surrounding inclusive education spaces for refugee background people with disabilities was one way to instigate change and create conversation (Noffke \& Somekh, 2011).

Feminist theory was important when considering the ethical implications of my research. I grounded my research in feminist theory as I was committed to undertaking research for and with my participants. I was conscious that my decision to interview people who worked with refugee background people with disabilities, rather than refugee background people themselves, could 'other' refugee background people. However, the stories of people who develop policies and educate refugee background people with disabilities are also necessary to facilitate change and analyse the strengths and weaknesses of inclusive education spaces and guide current and future policies and practices in this area. I made sure that all knowledge was treated with respect and dignity, and aimed to ensure that no forms of knowledge were 'silenced' by my research (Catlett \& Beck, 2007).

\section{Positionality}

It is necessary to recognise my positionality in this thesis. My experiences as a Pākeha disabled woman shape my interest in and responses to research into the interplay between geographies of disability and feminism. As one aspect of my disability physically manifests through facial palsy (which in turn stems from cerebral palsy), I strongly identify with the idea that my body is a visible space where my disability and femininity interact. This is a key idea found in both feminist theory and geographies of disability which rely on the subjective; the idea that every individual has different interactions with society. A key aspect of feminist theory which is relevant to my methodology is the idea of difference. Difference can be biologically constructed, but also can be socially constructed (Gatens, 1996, 1999). Acknowledging difference is essential when analysing results of interviews discussing inclusive education spaces. Examples of differences between myself and the researcher include age, gender and disability. Feminist geographies particularly focus on the connections between people, which can include acknowledging inclusion and exclusion of different societal groups (Dias \& Blecha, 2007). 
Despite experiencing societal exclusions (due to my disability and gender), I am still in a privileged position within the 'minority' of disabled, Pākeha women. I have the benefit of a strong academic background, and I am also a descendant of colonial settlers in New Zealand. My race and academic background mean that I have experienced race and class privilege in a (slowly transitioning to) post-colonial New Zealand society. I need to be aware of this privilege when conducting research. Additionally, using elements of Western thought to discuss my research results can perpetuate dominant voices (Gatens, 1999). All too often, minorities are further oppressed by passive research which disempowers, and further oppresses them (Dyck, 2000; Hall \& Kearns, 2001; Sultana, 2007). As I had experience of receiving inclusive education throughout my primary, secondary and tertiary schooling, it was important to acknowledge that this experience has influenced my knowledge and discussion of inclusive education spaces (Titchen \& Hobson, 2011). Scott (1997, p. 160) also emphasised that using 'familiarity devices' can help to establish trust and 'ease the process of entry' into participant spaces. Using my experience as a person with a disability helped to establish my credibility and authenticity when researching inclusive education spaces for refugee background people with disabilities.

Although I am a disabled researcher, I cannot speak for all people with disabilities, or ignore my privilege as an academic. In addition, it is important not to assume that all individuals with one disability have the same experiences. Imposing judgement upon people's experiences and reactions towards and of disability can devalue empowerment and marginalise the disabled, as it negates individual experience (Chouinard, 2000; Dyck, 2000).

Part of this research involved my awareness of potential bias that could be imposed upon participants. I also was impartial in interviews, as my own personal experience of inclusive education was not being investigated. However, as this thesis was based upon a social constructivist approach, I was aware that my positionality would affect the research process and acknowledging my positionality within research is therefore important (Creswell, 2014; Sultana, 2007). 
I did not fully anticipate the effect that my positionality as a disabled woman would have upon research interviews. As I outline in Chapter Four and Five, I was aware that my personal background and my positionality may have influenced participant answers to some questions (Creswell, 2014). Some participants thought that I had the 'right' definitions of disability and inclusion, and asked me for my opinion when they were questioned. Some participants were awkward when giving their definitions, which could suggest that they felt uncomfortable answering. Participant definitions of disability and inclusion may have been different if a non-disabled researcher carried out this research.

\section{Qualitative methods}

My research was conducted using qualitative methods because my research involved exploring aspects which could not easily be quantified and required rich, detailed data about experiences and practices (Creswell, 2014). The interview questions were also subjective, and allowed participants to share their experiences and ideas surrounding inclusive education for refugee background people with disabilities. Furthermore, using qualitative research was necessary as I was asking participants about their experiences relating to inclusive education spaces (see Appendix Three). As many of these questions touched on aspects of emotion, feelings and impressions, a qualitative approach was more appropriate than a quantitative one. Qualitative research also allowed me to explore the different nuances involved with experiences, and draw out deeper meaning that may not have been present in numerical data. Using a qualitative research method allowed me to develop deeper understandings and meanings of inclusive education spaces (Mthethwa-sommers \& Kisiara, 2015; Scott, 1997; StewartWithers, Banks, McGregor, \& Meo-Sewabu, 2014).

A qualitative research approach also allowed me to analyse participant answers based on their location and their occupation. My research is bounded by time, as it only looks at participant views on inclusive education during the period of August September 2015. In addition, the current events surrounding my research at the time of data collection meant that the data collected could have been influenced by media 
discourses surrounding refugee background people which were quite heated during 2015 in New Zealand and Australia.

Qualitative research was also useful for my research as I was able to tease out nuanced themes presented by participants as a result of the semi-structured interviews with participants (Creswell, 2014). This enabled my research to be at least partially shaped by the participants' priorities. Before I submitted my ethics application, I met with a couple of future participants involved in inclusive education and/or refugee background people with disabilities. The initial discussions with them enabled me to develop research questions based on themes that arose in conversation. However, as I did not know who all of my participants would be at that time, I was unable to assess whether the questions were appropriate for all participants until the interviews took place.

\section{Selection and invitation of participants}

I identified potential participants by emailing contacts who worked in the refugee sector in New Zealand. These contacts were either provided to me by colleagues, or suggested by participants themselves. The sampling strategy chosen was purposive, as I used my knowledge of the inclusive education and refugee background sectors to find participants who could represent the population (Berg, 2012). This purposive sampling was enabled by opportunities to access participants in Auckland and Wellington, New Zealand, and Melbourne, Australia. Melbourne was chosen as an interview location, as I was asked to present at the Inclusive Education Summit in JulyAugust 2015. I decided to make the most of the opportunity to interview participants in Melbourne, as I thought that their comments could help aid the discussion of what was and wasn't present in inclusive education for refugee background people in New Zealand. Australia was a good point of comparison against New Zealand. Interviewing Australian participants was also one way of evaluating New Zealand's current policies surrounding inclusive education for refugee background people with disabilities. Australia accepts more refugees each year due to its larger size, and therefore has a larger government and non-government organisation supporting them. Consequently, the interviews with Australian participants could suggest different aspects of inclusive 
education for refugee background people with disabilities that weren't initially discussed in New Zealand.

To find participants in Melbourne, I found relevant organisations to email, and requested an interview time while I was in Australia. I invited participants from a range of backgrounds who had expertise related to refugee background people with disabilities and inclusive education as they were working in a non-governmental organisation, an education provider, or within a government organisation.

I ensured that I interviewed a wide range of participants in order to gauge a general idea of discourses occurring within the inclusive education sector. The participants did not necessarily come from an inclusive education background, which reflected the diverse nature of the sector. Chadderton and Torrance (2011) emphasised that boundaries needed to be drawn in order to determine what knowledge should be included and excluded from research. Drawing boundaries in research is necessary in order to understand what is being discussed - inclusive education. Participant understandings of inclusive education were analysed in wider social contexts. Doing so showed how this research engaged with wider discourses surrounding inclusive education.

In total my study involved eleven participants in ten interviews. In one interview, two people were interviewed together at their preference - EP3 and EP4. Four participants came from Melbourne, Australia; three from Wellington, New Zealand; and four from Auckland, New Zealand. Of these participants, two were from government organisations (GO), five were from non-government organisations (NGO), and four were from education providers (EP). Only education provider participants had direct involvement in inclusive education through their work as teachers. The acronyms GO, NGO, and EP are used when describing results in order to protect participants' identities and ensure confidentiality. A number alongside each acronym is used to indicate different individuals (see Table 3.1). 
Table 3.1: Description of participants

\begin{tabular}{|l|l|l|l|}
\hline $\begin{array}{l}\text { Descriptor } \\
\text { used in } \\
\text { research }\end{array}$ & Affiliation & $\begin{array}{l}\text { Location (New } \\
\text { Zealand (NZ) or } \\
\text { Australia (AU) })\end{array}$ & Gender \\
\hline EP1 & Education - support & Auckland, NZ & Female \\
\hline EP2 & Education - teaching & Melbourne, AU & Female \\
\hline EP3 & Education -teaching & Auckland. NZ & Female \\
\hline EP4 & Education - support & Auckland, NZ & Female \\
\hline NGO1 & Non-government - service & Melbourne, AU & Female \\
\hline NGO2 & Non-government - service & Wellington, NZ & Female \\
\hline NGO3 & Non-government - advocacy & Wellington, NZ & Female \\
\hline NGO4 & Non-government - policy & Melbourne, AU & Male \\
\hline NGO5 & Non-government - service & Melbourne, AU & Male \\
\hline GO1 & Government -policy & Wellington, NZ & Male \\
\hline GO2 & Government - policy & Auckland, NZ & Male \\
\hline
\end{tabular}

In order to comply with ethical constraints and to maintain participant confidentiality when discussing their views on inclusive education with me, I redacted people's names and those of their organisations. For example, EP2 indicates that the participant came from an organisation which was an education provider, and they were the second interview transcribed and coded. As I received ethical permission to name the geographic location of the participants involved, I have included this information.

In writing up the research, I occasionally altered some of the data to protect participants and the refugee background people to whom they referred. For example, some participants mentioned instances where they had worked with refugee background people with disabilities, or described a refugee background person's specific disability and gender. As the disability communities in Melbourne, Wellington and Auckland are 
relatively small, I have at times redacted the refugee background person's gender and (where possible) disability. This ensures that the confidentiality of the refugee background person and the research participant are both maintained.

\section{Data collection and analysis}

I used semi-structured interviews to generate research data. Prior to the interviews taking place, I prepared a list of semi-standardised questions to ask stakeholders from government organisations and non-government organisations. These questions are found in Appendix Three. Initially I also prepared questions for refugee background people with disabilities, but did not find any to interview. These questions were provided to participants prior to the interview via email. Semi-structured interviews also enabled me to access participants' previous experiences of inclusive education, and participant experiences to which I wouldn't normally be privy. Semistructured interviews gave me insight into classroom situations that I could not observe directly (Scott, 1997).

Interviews were semi-structured in order to investigate what stakeholders thought and felt (Stewart-Withers et al., 2014) about inclusive education for refugee background people with disabilities, and to share knowledge between us. I endeavoured to use grounded strategies (such as follow-on questions) when interviewing, to allow my analyses of inclusive education spaces to be rooted in the participants' experiences (Barbour \& Schostak, 2011). These questions endeavoured to engage participants in a discussion surrounding inclusive education spaces for refugee background people with disabilities.

Participants chose which interview questions to answer. Not all questions applied to all participants, so some chose not to answer some questions based on their experiences. The length of each interview ranged from 25 minutes to one hour and ten minutes depending on the time people could give me. All interviews were recorded, and all took place in an office space chosen by the participant. I travelled to the participant's preferred work location for every interview, as all interviews took place during working hours. Each interview was transcribed, but was not shared or modified prior to analysis, 
due to time constraints. Unfortunately, upon the completion of my thesis, I discovered that I had included the clause that interview transcripts would be shared with participants before analysis. Not doing so was an error on my part, and I have apologised to participants for this oversight. However, I ensured that all participant views were accurately portrayed in my analyses.

The nature of the questions mean that I was not endeavouring to generalise participant answers or suggest that they were reflective of the wider population. I judged that the questions that I was asking indicated the individual's understanding, and not necessarily that of the organisation they worked within (Chadderton \& Torrance, 2011).

Once all ten interviews were transcribed, I assigned a relevant acronym (GO, NGO, EP) and number to each participant. I then went through all transcripts using an inductive process; organising the data into broad themes (Creswell, 2014), and coding data using a thematic analysis that relied on the original research questions. Berg (2012) described themes as a simple way of analysing content within data, and extracting information. I particularly used directed content analysis, which involves the use of themes derived from existing theories that relate to the research. I immersed myself in the data, pulling out themes that had already been identified in the research question, and excavating the interview transcripts to find themes that weren't already obvious (Berg, 2012).

The three key themes that I identified were contested definitions of disability and inclusion, multiple layers and dimensions to inclusion, and barriers and challenges of creating inclusive education spaces for refugee background people with disabilities. The themes were derived from feminist theories and models of disability identified in the literature review. Once these three themes were identified, I used deductive reasoning to match the data to the themes. Therefore, the process of analysing data was thorough as an inductive and deductive process was used (Creswell, 2014). I also administered a reflexive approach to the data, in order to understand the effect that my positionality had upon participant responses (Creswell, 2014). 
As this research was an intersection between geographies of disability and education, it felt 'right' to have an intersectional approach and use different aspects of qualitative methodologies. Throughout the course of this thesis I've discovered that methodologies are generally messy, particularly when delving into the world of subjectivity, knowledge expertise and creation. However, this messiness is what makes qualitative research so suited to studying human concepts; as humans, we are messy.

\section{Ethical considerations}

There were many ethical considerations debated when undertaking this thesis. One consideration was whether to interview refugee background people with disabilities, or limit my interviews to policy makers and service providers. In the end I chose not to interview refugee background people with disabilities primarily due to the sensitive material that would be discussed, and my awareness that it takes time to build up trust with an interviewer, and then disclose one's experiences of disability, and refugee-related experiences. Although my research aimed to benefit refugee background people, it was not necessary to interview these people for my benefit, and the research's benefit. I was aware that the majority of refugee background people with disabilities came from different cultures, and disability could be perceived negatively within these cultures. This negative perception could have affected a participant's willingness to disclose their disability and describe their experiences of education to me.

Secondly, I did not want to 'force' a refugee background participant to discuss disability as a form of identity, particularly if they were coming to terms with disability themselves. Although I have a positive view of disability, I did not fully accept my disability until I was in my early twenties, and I am still negotiating my identity through disability to this day. I was also aware that I would not know the refugee background people with disabilities prior to interviewing them, and discussing personal experiences and the effects of disability could potentially create an uncomfortable atmosphere, where I would be 'extracting' answers from participants. If I was in the participant's situation, I would not want to disclose details of my impairments to someone with whom I had previously had little contact. 
This research received approval from Victoria University of Wellington's Human Ethics Committee (Approval number 21846). The research process was thoroughly vetted prior to beginning interviews. Participants received copies of the interview information sheet (see Appendix One) prior to the interview. Some participants also requested to see the interview questions (Appendix Three) prior to the interview, and these questions were emailed to them. They were also given time to read and discuss the consent form (see Appendix Two) prior to the interview formally beginning. All participants indicated that they wished to receive feedback from this study. They will receive this feedback at the culmination of this thesis.

\section{Limitations}

There are a number of limitations to this research. A major limitation to this study was time. I only had one year from the time of enrolment to develop, research and write a comprehensive thesis. If more time was available, I would interview more people from different organisations in order to have a broader, more holistic range of data to analyse. I would also consider mixed methods approaches to research in order to gather more information which could be used to evaluate current practices.

A significant limitation to this study is the small sample size for this thesis (only eleven people). This sample size was due to limitations of purposive sampling. Purposive sampling was also a biased method of obtaining participants, as I relied on my personal knowledge about inclusive education and refugee background people to select interview subjects (Berg, 2012). This limited the amount of people that I interviewed, as there were some people that I was unaware of that could have provided valuable insights. I was only able to interview one person from each organisation that I approached, apart from one interview. A further limitation was my reliance on semistructured interviews to identify participant views. Using interviews meant that my study was heavily focused on the present day. However, my research looked at current inclusive education spaces, and how these could be improved, so focusing interviews in the present was appropriate. I also asked questions which asked participants to consider past experiences, or their ideal for the future (Chadderton \& Torrance, 2011). While the results are not generalizable, they do give insights into each of these sectors, and my analysis also sheds light on some of the key themes which research in this area entails. 
One possible use of this research in the future is that the findings could inform a wider sample of organisations involved in refugee education and policy as well as schools.

I only had a year to interview, disseminate results and write a master's thesis, which was not an adequate amount of time to develop rapport with potential participants. If I had more time, I would consider interviewing refugee background people with disabilities and incorporating their ideas into research questions for service providers, government workers and policy makers. Changemakers Refugee Forum (2016) states guidelines for working with refugee background people during research, and these guidelines were also what led to my formative decision not to interview refugee background people with disabilities. I was aware that people with disabilities and people from refugee backgrounds can be used in research for tokenistic reasons, and it would be naïve of me to use one person's experience as a foundation for all refugee background people's education experiences.

Another limitation of this research was my inexperience in this sector which is shaped by confidence and knowledge. This influenced my responses to participants during the interview. For example, I felt in awe of some of the participants, and let a couple of participants dictate the majority of the interview. Looking back on the transcripts of these interviews, I found the participant responses harder to distil as the structure of other interviews was not followed. Interviews were also unconsciously influenced by my thoughts and emotions of the particular day. Although I endeavoured to 'leave' my thoughts and emotions at the door in order to concentrate on the interview, sometimes nerves overtook reason. I felt more comfortable with participants who I had previously met, which may have allowed our interviews to progress to deeper levels of analysis than people I did not know. I was particularly nervous when I conducted interviews in Melbourne. It was the first time that I had undertaken interviews, and I was often flummoxed from navigating transport prior to arriving. These experiences inevitably shaped the quality of the data which needs to be acknowledged. 


\section{Summary}

In summary, this chapter has described the feminist theory and social constructivist perspective upon which this research was based. The epistemological framework that I used meant that I was able to critically distil the social constructions surrounding disability, inclusion and inclusive education for refugee background people. I discussed my positionality and outlined the qualitative methods used in this research and how I involved the participants in semi-structured interviews. The data that emerged from the participant interviews provided a rich tapestry for thematic analysis. However, the data could have been affected by my positionality and my consequent relationships with participants. The ethical considerations and limitations of this research could also influence my results, although all research raises the question of 'what if...'. The next two chapters detail the research results and corresponding discussion which emerged from my methodologies. 


\section{Chapter Four: Participants' perceptions of inclusion, disability and inclusive education spaces}

\section{Chapter overview}

The focus of Chapter Four is to answer my first research question stated in Chapter Two, which was 'what are the definitions and interpretations of inclusion, disability and inclusive education held by key stakeholders in refugee support, education and policy?' In this chapter, I discuss how the participants defined inclusion, inclusive education and disability, and how these definitions shaped their perceptions of inclusive educational spaces. Participants' definitions are important to focus on as they provide insight into their knowledge and perceptions of the above concepts, and therefore how they, as people involved with refugee background students, implement inclusive education spaces.

Knowledge and theory about concepts generally affects practice (praxis) (Cahill, 2007; Freire, 1993). In this chapter, I distil key concepts from participant responses, and link these responses to wider literature and theory. The definitions varied between participants, which suggests that there were different levels of understanding of the terms discussed. While there was some degree of commonality in definitions, variations could be attributed to participants' occupations, previous experiences of refugee background people and/or people with disabilities, and their locations (in New Zealand or Australia). In this chapter, I reflect upon and examine these difference and similarities in relation to the literature and theories introduced in Chapter Two.

\section{Defining inclusion and disability}

Definitions of inclusion and disability were contested among participants. As stated in Chapter Two, one reason for the ambiguity of definitions could be because the concepts are abstract and not easy to pin down. They are also political and contested in society. The majority of participants were also asked these questions on the spot and may have had little time to consider the definitions carefully. While there were significant differences between education providers, and stakeholders from government 
organisations and non-government organisations, it seemed that having personal experience of interacting with people with disabilities probably contributed to the most thoughtful responses. These answers aligned closely with the social model of disability.

Most participants were uncertain and tentative when providing definitions of disability and inclusion. All participants paused before giving definitions. As stated in Chapter Two, different concepts and definitions of disability exist and these are widely contested. This can create confusion among people who work in the disability sector over what is the 'right' definition and model to follow (Gable, 2014; Šiška \& Habib, 2012). Gable (2014) also stated that reductionist definitions of disability were not possible owing to the complex nature of the concept. Participant confusion and uncertainty could be because it is difficult to reduce disability and inclusion to succinct definitions (Gable, 2014). Consequently, my questions may have appeared presumptuous, assuming that participants had a 'correct' definition of disability and inclusion. In the following sections, I outline how inclusion and disability were defined by analysing responses from the three different sectors involved in the research Education Providers (EP), Government Officials (GO) and Non-Government Officials (NGO).

The two government officials interviewed struggled to fully construct definitions of disability. GO1 stated that "it should be a simple question", and was hesitant in forming a complete answer. They stated that disability was

"I guess anything that um... impedes someone from being able to uh... I don't know, there are things in terms of mental health or other forms of disability... sorry I haven't really got a definition".

This answer suggests that disability was not at the forefront of their work. GO2 was clearer in his definition of disability, but also stated that it was "a good question". He said "I think it's anything where the person is not able to fully participate in the community, so it can be physical, or mental, but the issue stops them participating in a way that's meaningful for them". He also saw disability as a "range of things", indicating that there was a variety of disabilities. GO2 also identified that disability was "sometimes as much about perception as it is about physical or mental impairment" 
indicating associations with a social model of disability. GO2's answer showed indicated that his organisation had more involvement with disability than that of GO1.

Government participants provided mixed definitions of inclusion. GO1 said that "inclusion is sort of synonymous with participation. Being able to take part, and wanting to take part". This definition introduced a new idea about inclusion which moved beyond equality. GO2 also mentioned that inclusion was where "you can participate to the level that you feel comfortable with and that you want to". He stated that inclusion was a "two way interaction", indicating that inclusion was based on people's wants and needs to be incorporated into a process. and make a person responsible for managing their disability (Gleeson, 1999; Thomas, 2004).

NGO participants discussed equality, participation and power in relation to inclusion. NGO4 simply stated that inclusion meant that "there are equal opportunities for people to participate, there's no discrimination or isolation of people". NGO3 described inclusion as "people are feeling that they are part of something [...] they are there because it's their right, because they're human beings". She also mentioned that inclusion was "a power thing". This indicated a recognition that inclusion was sometimes experienced by people who were "allowed", which suggests that there is still the possibility of exclusion, as one person had the power to include the other. $\mathrm{NGO} 2$ had a similar definition to NGO3, saying that inclusion was "regardless of race, ethnicity, religion, sex, disability, all of that. For me that would be inclusion'. NGO5's definition of inclusion included disability, stating

"regardless of what disability you have, you have to be included. That is something we need to advocate for those people in terms of what disability they have".

He went on to say that "sometimes people with disabilities have strange behaviours themselves", and appeared to view people with disabilities as different, despite his earlier assertions of including everyone. As discussed in Chapter Three, these ideas stem from the charity and medical models of disability which both viewed a disabled individual as an anomaly, someone abnormal (Dear et al., 1997; Gleeson, 1999). The use of the word "strange" suggests that people with disabilities are still perceived in a 
different context to able-bodied people, and begs the question: do able-bodied people have strange behaviours too?

There were a number of other statements by NGO participants which showed their perceptions were underpinned by the medical model of disability. NGO1, said

“I don't know, because there's lots of different kinds of disability, ones that you're born with and require [things] and there's ones which make it difficult to do things but are totally curable”.

NGO1's references to "curable" reinforces the medical model's idea of disability being a treatable condition that an individual has (Gleeson, 1999). NGO1 also stated that "I guess all disabilities are totally manageable, but in my client's case he's disabled because right now he cannot live life to the fullest". These statements demonstrated NGO1's background as a support worker in a service, as disability was perceived as "manageable”. Participants from NGOs also stated that disability was a social construct, and most made the distinction between acquired and congenital disabilities. NGO5's interpretation of disability emphasised the idea of disability as visible difference. He stated "I can tell how they look, and their body posture, the way they talk and walk, their presentation. I can identify what disability they have". However, he also stated

"I can't formally judge if he or she has a certain type of disability. But I am able to feel disability; intuition. I think that's how people also find out there's something wrong with their kids".

This statement indicates that people are able to judge disability based on experience. It also demonstrates that NGO5 saw disability as a negative construct as it was something that was "wrong with their kids". His comment highlighted that there was a hierarchy between people with disabilities and able-bodied people (Connor, 2013; Worth, 2013). This hierarchy echoes the medical model of disability and the idea of disability as a negative aspect of the individual and society (Gleeson, 1999).

Some NGO participants linked disability to culture, an approach which is underpinned by the social model of disability. When discussing culture, participants generally defined culture broadly and used singular terms to describe western and non- 
western cultures. This indicated that there was a clear divide between western culture and all other cultures which did not derive from a western outlook (Gable, 2014; Hassanein, 2015; Šiška \& Habib, 2012). Gable (2014) stated that disability could be interpreted as a socio-cultural construct owing to the effects of different culture upon an individual's impairment. NGO3 stated that "in some ways it's like cultural [...] if people have a specific disability then they have to operate in a particular way”. She also added, "it's a kind of cultural difference, just trying to understand where they are coming from in the same way as working cross-culturally”. These cultural aspects arose from the different circumstances people with disabilities could be born into. NGO3 also stated that "if you've had to learn things differently, and organise and think about life differently, it's a different worldview'. NGO3's comments on disability being a different aspect of culture and worldview linked to NGO2's comments on disability as a "different way of being to what people think is generally [the norm]". NGO2 also explained that disability was "someone's different, like you and I are different". This statement demonstrated that disability is a measure of difference in society. The link between disability and culture is interesting, as it suggests that refugee background people are considered to be a homogenous cultural group. Culture is also used as a broad term to convey embodied difference. It raises the question of whether it is more acceptable to discuss culture rather than bodies.

NGOs and GOs who worked in service provision or policy tended to use more economic terms in their definitions of disability and inclusion. This suggests that these participants dealt with funding issues more than the education provider participants. The use of economic terms could also indicate a level of professionalism and distance between the service provider and the disabled person (often referred to as the 'client'). These economic terms used could link to the charity model of disability, where people with disabilities are seen as 'needing' to be looked after. Economic terms also link to the medical model of disability, as often service providers needed to classify a refugee background person's disability before receiving funding. Classifying a person's disability can also dehumanise the experience of disability.

Overall, education provider participants had the most nuanced and decisive definitions of disability and inclusion. Their definitions were perceptive and reflected 
their daily interaction with people with disabilities. Education providers stated that inclusion involved providing equal opportunities for individuals in the classroom. "To be able to participate, to contribute, to be involved in any form or shape, in any activity or project. For me that's inclusion" EP1 stated, indicating that inclusion was the holistic integration of students in the classroom environment. This definition was echoed by all four education providers. The education providers' definitions mirror most closely the ecological model of disability, which emphasises the integration of the individual and the environment (Ebersold \& Evans, 2003) (see Table 2.1 in Chapter Two). EP2 stated that

"ultimately I think that true inclusion happens when no one notices what's happening, and everyone has equal access to education and employment [...] everyone's coming to it on equal footing including extra support needed".

Her definition included education and employment, indicating that inclusion was a broad concept and practice that transcended formal contexts and policies. EP3 and EP4 weren't asked directly to define inclusion, but the concept was discussed in relation to former students. Generally, their definition of inclusion matched EP1 and EP2.

All education providers' definitions of disability indicated that disability was a societally-derived construct, and not necessarily derived from individual experiences and conditions. EP2 stated

"my go-to definition of disability is really the definition that's in the Convention on the Rights of Persons with Disabilities, which basically doesn't define disability, it defines people with disabilities".

Her definition of disability incorporated the social model of disability. This definition may have been more 'academic' due to her positionality and employment. EP2's definition directly referenced the wording found in the United Nations Convention of the Rights of People with Disabilities which defines people with disabilities as

"those who have long-term physical, mental, intellectual or sensory impairments which in interaction with various barriers may hinder their full and effective participation in society on an equal basis with others."

(United Nations, 2006). 
She was the only participant who directly linked her personal definition with the United Nations. EP2's definition also hinted at the ecological model of disability, as her definition, and the United Nations definition, considers both the role of society and the individual in disability (Ebersold \& Evans, 2003).

All education providers discussed the relationship between different disabilities and the socio-spatial environment. EP1 stated that "disability can be visible but can also be invisible”. She gave examples of visible and invisible disabilities in her definition. She stated that visible disability could include "wheelchair users, could be people who suffer from a physical condition". The use of 'suffer' indicates that disability could be perceived in the rather old-fashioned charity model. However, EP1was also aware of the deficit nature of her statement and also added that "it's really an impairment that prevents the individual from being able to function as normally, or actually I don't like to use the word normally, it's not a very good word." This showed more of an awareness of the effect that language had upon definitions of disability, and the effect that language could have upon 'othering' people with disabilities.

EP1's definition also related to EP2's as she said that "society makes people feel disabled. It is the society's understanding of what normal people experience, and they use that standard to define that whole model of disability". The mention of the social model of disability indicated that EP1 and EP2 had a theoretical understanding of disability, as well as practical, hinting that they understood how theory links to praxis (Gable, 2014). The two other education providers, EP3 and EP 4, also primarily relied on social understandings of disability: EP4 said

"I think society is the disability in terms of what's put on people with a disability [...] it's all round the wrong way, how society perceives other people that's where the disability comes in".

She emphasised that the definition of disability is a "hard one", and that "society says and puts that disability onto people".

EP3 linked her definition of disability to refugee background people, saying "it's often not their problem at all, it's a lack of opportunities and experience. So 
you've got to be very careful about whether you're making a judgement". The inclusion of refugee background people in EP4's definition of disability suggests that societal perceptions of disability can have negative consequences for refugee background people with disabilities (Šiška \& Habib, 2012).

While this analysis above demonstrates how employment positions in society (GO, NGO and EP) shaped perceptions, three participants across these sectors' definitions of disability and inclusion stood out as they were shaped by personal experience (EP2, EP4 and NGO1). Personal experiences can influence perceptions of disability and inclusion, and frame individual interactions with the two concepts (Dyck, 2000; Goodley \& Runswick Cole, 2014). Participants explicitly made this link in their interviews. EP2 had a sibling with disability, which influenced her understanding of inclusion. She said "I actually grew up in a disability community without knowing it [...] not knowing that I was existing in an inclusive world". EP4 (who had a child who some would define as 'disabled') said:

“Our [child]'s been given labels and they absolutely refuse to take them on board, and we didn't bother getting them assessed for everything we were told to. And we, they, have support, they have free will and they're really resistant to labels, and I can get that I think".

Inclusion was considered to be a "buzzword" by NGO1. She also incorporated personal experience in her definition, saying “it's weird for me because I have a disabled brother, so disability for me is really not an issue, like it's not something that you think about as being different". She also said that "for me this is just normal for disabled people to be included in the same way as everyone else".

These statements suggest that personal connections with disability can influence definitions and perceptions of inclusive education. Feminist theorists discuss how embodied insights can also influence relationships to space and society (Gatens, 1999). Worth (2008) and Chouinard (2000) also discusses how personal experiences can be political. Chouinard (2000) suggests that personal experience of disability helps to frame and critique societal understanding of disability. NGO1, EP2 and EP4's 
connections with disability help them to develop an understanding of how the 'other' is perceived in society and as a result held wider and more inclusive definitions.

Two participants also expanded upon their definitions of disability after asking for my opinion on disability. NGO3 and NGO4 asked for my definition of disability, and GO1 asked "what's the good answer there?" This question suggests that I was considered to be the more knowledgeable person on disability definitions, and participants did not want to present the 'wrong' answer. As a disabled woman, my positionality was also emphasised through being asked these questions. Personal experience is significant to research within geographies of disability, as disability scholars generally have a personal connection with disability (Worth, 2008). The participants assumed my personal experience to be authoritative, when it wasn't necessarily so. Deferring their answers to me also could have emphasised insecurity or a lack of confidence when providing definitions. Disability geographers and feminist disabled researchers discuss how personal experience is politically charged - a way of comprehending processes of discrimination and exclusion, and how they are perceived by others (Crooks, Dorn, \& Wilton, 2008; Garland-Thompson, 2004; Worth, 2008, 2013). Being asked for my opinion of participant definitions emphasised how the personal is political, and emphasises the importance of recognising my positionality in my methodology. It also allowed me to understand that my disclosure and identification as a disabled woman could influence participant answers to questions. This made me consider what discussions could have arose if I had not presented myself as being disabled.

\section{Defining inclusive education}

As well as asking about inclusion and disability, I asked participants to define inclusive education. As stated in Chapter Two, an inclusive school was described by Curcic et al as 'those that adhere to democratic principles, including collaborative decision-making and practice, celebration of diversity (including ability diversity), engagement with the broader community, a curriculum relevant to students' lives and flexible learning contexts' (Curcic et al., 2011, p. 119). Participant definitions of inclusive education and inclusive education for refugee background people with disabilities echoed Curcic et al's definition, however, rarely in its fullness. As with the 
previous section, there were some differences in participants' responses and the confidence of participants when providing definitions. Education providers generally gave more nuanced definitions than government and non-government workers, and also hinted at education spaces in their answers. These definitions also relate to wider conversations about models of disability and inclusive education and debates about mainstreaming versus the provision of separate specialist facilities.

Generally, most NGO participants' responses indicated uncertainty with the definition of inclusive education. New Zealand participants from non-governmental organisations generally defined inclusive education as incorporating a range of learning methods. NGO2 was critical in her definition of inclusive education, saying "so for me inclusive education isn't necessarily how we see the education system today unfortunately, because it isn't really accessible”. She said:

"There're people feeling isolated, not grasping what's happening, and that's because we have a certain way of delivering education. So for me education would be taking into consideration a variety of learning methods and delivering based on that".

Her statement illustrates the need for improvement within inclusive education practices. NGO3 indicated a sense of uncertainty and lack of confidence in her definition of inclusive education, as shown by her frequent use of "um". She stated that inclusive education was "where there's a place for everyone. So everyone, um... everyone's valued and um, that social, emotional and intellectual development to the best of their capacity". The participants from government organisations were also both unsure of how to define inclusive education. GO2 stated that he was unsure, saying "I don't know, to be honest". It was clear that inclusive education was not within the general scope of his occupation, as he said "if I had to define it, I would probably say it's about being able to make sure that people are actually part of an education system that has a full range of participants in it, rather than having it too specialised". GO1's response also indicated that his professional scope did not include inclusive education. His statement began awkwardly, as he stated "um... education that doesn't... that takes account of all nuances of the individual, their need and perception of where they're at. Taking account of each individual's learning needs, their backgrounds and all the rest of that". Like GO2, GO1's response was generic, and did not specifically reference people with 
disabilities or refugee background people. Both GO1 and GO2 mentioned exclusion as a key aspect of the definition of inclusive education. GO2 said "so that no one feels excluded from participating based on a range of factors". Similarly, GO1 said that part of inclusive education involved "people feeling connected, and not being singled out for, or cast aside because they're being treated differently or being excluded from activities".

Non-governmental organisation participants generally did not have as much personal experience with inclusive education as education providers, and as such, did not have such clearly thought-through definitions. NGO5, from an Australian NGO, said that inclusive education was "something I need to think about". He linked defining inclusive education with different service provisions and different spaces which could be created to foster inclusion. He stated that "we have different sections within service, for example youth services, and youth services have lots of inclusive education things".

NGO5 also linked inclusive education with service access, saying "each individual will have to understand service access and go through the same processes, this will become more common for people with disabilities". Having access to employment was also considered important for people with disabilities and inclusion in society. NGO5 stated that

"we understand that they can't do much but they have to do something [...] if we utilise someone's autistic abilities he or she can work really well in factories or places that support their development".

Although this quote was well-intentioned, there was an undercurrent that people with disabilities were 'beneath' abled-bodied people. This related to the hierarchy of disability discussed in Chapter Two (Crooks, Dorn, et al., 2008).

NGO1, also Australian, was also unsure how to define inclusive education. Her initial answer was “God, I don't know. Educating people inclusively? ” Her comment suggests that she was uncomfortable with being asked to define inclusive education. She followed her initial statement with "every one of different abilities are catered to. Things are flexible, there's not a rigid way of doing something. Everyone's needs are 
respected and managed". Unlike NGO1 and 5, who were service providers, NGO4, as a policy maker, simply said that inclusive education was "involving the students in their learning and in the direction of their learning. And also [...] equality in education across the classroom regardless of impairments". All three participants referenced disability in some form within their definitions of inclusive education. These references to disability also demonstrate links between participant responses and the medical and social models of disability discussed in Chapter Two. Inclusive education was discussed in relation to societal inclusion, but there was also a sentiment that an individual was also responsible for the management of their disability (Gleeson, 1999; Thomas, 2004).

As educational practitioners, educational providers had more direct experience of classrooms and curricula. They were able to give more specific answers, as well as more general responses about inclusive education providing every single individual with the opportunity to participate in education. EP1 stated that

"inclusive education refers to the education that allows every single individual regardless of their backgrounds, their ability or disability to be able to participate freely and to receive educational opportunities".

This statement generally described the overall definition of inclusive education for all four education providers. EP2 identified choice as a presiding factor of inclusive education. She stated that "My stance really has been that inclusive education is largely about choice [...] so choice of the individual, and [...] I think desegregation is really important too". EP2's use of the word 'choice' indicated that an individual should have options surrounding their education. She stated that "[choice is] really the most important thing in a lot of rights protection areas, inclusive education being a right". This statement suggests that choice, and in turn inclusive education, are rights which should be accorded to the individual. EP2's remarks echoed EP1's remarks in a separate interview, where she stated that

"Involving the students in their learning and the direction of their learning, and also showing that all participants are able to equally learn and engage in education across the classroom, regardless of impairments". 
Involvement in education can provide students with the choice to determine the direction they wish to take (Curcic et al., 2011; Hassanein, 2015; Šiška \& Habib, 2012; Zembylas, 2013).

Participants emphasised that inclusive education involved including everyone. EP4 expanded upon her initial definition of inclusive education as "including everyone" to also include a discussion of rights, saying "In the classrooms or schools it's ensuring that everyone has the right to access all aspects of the curriculum, school life and the schooling”. This discussion linked with EP3's definition of inclusive education in the same interview; stating that "if we're talking about inclusion we need to include all in human rights". EP3 and EP4 also mentioned that inclusive education was broader than just considering refugee background people and people with disabilities. EP3 said that

“...for us, inclusive education perhaps becomes that much broader because we're working with people who've come from less inclusive environments. And for people who've become refugees because of difference so it might be religion or ethnicity. These sort of things we might not see as a disability but they have excluded people”.

This statement indicates that inclusive education needed to consider a range of factors that could have previously excluded people from learning. These statements from education providers suggest that there is more than one component of school life which delivers inclusive education. Schools are seen as places which enable students to prepare for future life, as well as participate in their education (Curcic et al., 2011). Teachers facilitate the students' learning direction and help to develop citizens which will make a positive contribution to society. These citizens are developed by making the school an inclusive education community (Curcic et al., 2011).

The different answers given by participants also show that inclusive education has several components to it, and that there is no clear definition. There are also many aspects to a definition which may only be known depending on the professional experience of a participant. Education provider participants were more likely to reference the curriculum in relation to inclusive education, as EP4 said "in the classrooms or schools it's ensuring that everyone has the right to access all aspects of 
the curriculum, school life and schooling”. In contrast, non-government workers emphasised flexibility and government workers tended to focus on student participation within schools.

\section{Provision of separate specialist facilities or mainstreaming in education}

Specialist education spaces compared to mainstream inclusive education spaces were briefly discussed in interviews. Desegregation was a difficult discussion point raised by EP2, as there are many contested ideas surrounding it in the literature (Kearney \& Kane, 2006). As mentioned in Chapter Two, Kearney and Kane (2006) discussed how inclusive education arose from changes to special education, although there were contested discussions whether inclusive education should only apply to mainstream schools. EP2 touched on the importance of desegregation within her definition of inclusive education. As the sole education provider interviewed in Australia, her definition of inclusive education differed from her New Zealand counterparts based on her inclusion of desegregation. She stated that:

"I don't think that there should be a law saying that [desegregation], I think that people should have the choice for segregated schooling if they want that, and I know it's really important for the Deaf community so [...] that should be respected".

This statement indicates the complexities surrounding inclusive education for people with disabilities. Some communities (such as the Deaf community) do not wish to be included in mainstream education. Consequently, inclusive education needs to work in the best interests of all communities, even if it results in the apparent exclusion of a community (Curcic et al., 2011). EP2 also stated that:

“I don't really get the argument for special schools or special schooling that have special education segregated within an educational facility. I generally think that's a bad idea. And that's from my personal experience as well as experience in research. There's a kind of stigma attached to that type of segregation”.

As well as indicating EP2's negative feeling towards segregated education, this statement emphasised the importance of personal experience in developing 
understanding of inclusive education (see earlier discussion on her personal experience of a family member with a disability).

Segregated schooling links to discussions of special education. Special education differs from inclusive education as it focuses on a person's medical diagnosis in relation to education (Connor, 2013). An individual's disability is the reason for alternative education being provided. However, inclusive education generally suggests that a person with a disability is included in all aspects of education, and not segregated from their peers. The comments from the education providers generally signalled a movement away from the caste-like system associated with special education, to a more inclusive, all-encompassing model of education. Generally, special education is associated with the medical model of disability, while inclusive education is associated with the social model (Gable, 2014). As the social model of disability also considers cultural factors, an inclusive education space would strongly benefit refugee background people with disabilities. Although EP2 found segregated education had negative effects upon students, Curcic et al., (2011)'s discussion of schools as communities suggests that there could be benefits to having similar people in education facilities. The 'hidden curriculum' allows students to learn how they are related to others from a young age (Curcic et al., 2011, p. 119). Segregated schooling may decrease the risk of marginalised students being excluded by their peers. However, inclusive schools should foster student preparation for future life through the school community, so a segregated school may not be reflective of wider society (Connor, 2013; Curcic et al., 2011).

Interestingly, while all participants advocated for inclusive and mainstream education, many mentioned that refugee background students had some specific needs, which could relate to the effects of broken educational histories and increased time and energy demands by family members during resettlement. This echoes Marlowe et al's (2014) and Taylor and Sidhu's (2012) belief that educational provisions for refugee background students primarily focuses on their psychological needs. Although no one suggested an entirely separate education system for refugee background people, some participants discussed how special treatment and processes needed to be in place to support refugee background students. Systems and transitions were important when 
implementing inclusive education for refugee background people with specific needs. GO2 stated

"I think this is part of the model they have for refugees, is that there might be transition to bring them in. Because you don't want a situation where someone, especially with English language, may feel that they're not doing well, and that has an ongoing effect".

He emphasised that transitioning into the New Zealand education system was important "because you don't want to set them [refugees] up to fail from the beginning. Or make them feel like they're going to fail because they can't speak or they can't participate". GO1 mentioned that inclusive education for refugee background people needed to be "mindful of their refugee backgrounds and their cultural norms and all the rest of that". He also mentioned that education needed to

"Take account of the psychological and emotional trauma that a lot of refugee background people have been through [...] there's certain things that need to be heard, certain things that impacts the way you interact with people that have numerous issues. I guess that's it, personally I haven't really thought about it."

This statement illustrated the perception that refugee background people required special consideration to achieve inclusive education. It also illustrates that inclusive education for refugee background people wasn't always considered. However, refugee background people needed "certain things" (GO1) in order to receive an inclusive education. The need for unique programmes for refugee background people was also emphasised by other government officials in Australia. NGO1 said

"I think that here in Australia, there are specific education providers for all refugees and asylum seekers, because there are so many. So I guess it's inclusive in the sense that they're designed specifically for people with no English, who've come from torture and trauma backgrounds, and who initially aren't able to access mainstream services."

Her statement emphasises the difference in education providers in Australia and New Zealand, due to the population differences. She also mentioned the importance of teachers in recognising issues which may be specific to refugee background people. "Teachers are generally more aware that they might be teaching people who have serious torture and trauma issues, as opposed to standard English language teachers" 
(NGO1). NGO1's statement introduces another level of care for refugee background people in inclusive education.

This level of care goes beyond most policy definitions of inclusive education. These definitions tend to be rigid and focus on inclusive education as a physical concept. Yet Zembylas (2013) discussed care for refugee background people with disabilities as a form of implicit activism in the classroom. Care as an implicit activism directly negates the effect of neoliberalism upon education spaces, and reinforces the idea of the student as an individual, not a commodity (Šiška \& Habib, 2012; Zembylas, 2013). The education provider participants particularly acknowledged how demonstrating care for students, and by students in the classroom had positive effects for refugee background people with disabilities.

\section{Summary}

The definitions of inclusion, disability and inclusive education, showed many similarities between education providers, government organisations and nongovernment organisations. However, differences were also apparent which related most specifically to different levels of involvement with refugee background through occupation as well as personal experience with disabled people in the three groups. Answers ranged in terms of surety.

Participants primarily defined disability and inclusion through social and medical models of disability. As discussed in Chapter Two the social and medical models of disability are the two main models of disability applied in society today. Both models are political, as they determine who has access to services, and to what extent (Chouinard, 2000; Gleeson, 1999; Swan, 2002). Most participants' understanding of inclusion generally followed the social model of disability; the idea that society is responsible for disabling individuals (Gleeson, 1999; Swan, 2002).

However, some participants made statements which suggested that they perceived people with disabilities as the 'other'. The education providers' discussion of 
inclusive education demonstrated the most inclusive and ecological definitions of inclusion and disability and showed the most carefully considered responses to the idea of inclusive education spaces. Their definitions reflected wider shifts over time in perceptions and obligations to people with disabilities in education.

Connor (2013) states that people with disabilities were once perceived as charitable cases, and had negative labels placed upon them by society. However, three participants showed that personal experience could be more powerful that occupational status when constructing more inclusive and ecological understandings of disability and inclusion. NGO1, EP2 and EP4's definitions of disability and inclusion were underpinned by an ecological model of disability; the understanding that it is the combination of the individual and the environment which creates disability (Ebersold \& Evans, 2003). Their definitions of disability and inclusion could have aligned with the ecological model owing to their personal experiences as family members of a child with a disability. This suggests that experience is very significant in this area.

Overall, these definitions suggest that inclusive education for refugee background people with disabilities involves a more detailed level of understanding of inclusion and disability than might be currently considered within government and nongovernment sectors. Having clear definitions of inclusion and disability can enable organisations to determine what is needed for refugee background people with disabilities to access inclusive education spaces - and these definitions are also political because they shape who gets what in terms of policies, funding and support (Chouinard, 2000; Gleeson, 1999; Swan, 2002). This chapter has built the foundations for the next findings and discussion chapter, which discusses how inclusive education for refugee background people with disabilities could be effectively designed and delivered. 


\section{Chapter Five: \\ Design and delivery of inclusive education spaces}

\section{Chapter overview}

This chapter discusses two further aspects of inclusive education: design and delivery. As discussed and defined earlier in Chapter Three, the concept of inclusion in education has a focus on both delivery of education (through pedagogy and programmes) and design of the built environment. Earlier research found that inclusive education design and delivery contributes to the wellbeing of refugee background students with or without disabilities (Holt, 2003; Komardjaja, 2001; Mthethwa-sommers \& Kisiara, 2015). In Chapter Four, I discussed participants' perceptions and theoretical understandings of disability and inclusion. Chapter Five now discusses more practical implications of designing and delivering inclusive education spaces for refugee background people with disabilities. This chapter answers research questions two and three: 'what are their perceptions of the challenges of implementing inclusive education spaces for refugee background people with disabilities, and how do they perceive ideal inclusive education spaces?' and 'how could participants' ideal inclusive education spaces help create a more holistic model of inclusive education for refugee background people with disabilities?'

In this chapter, I examine the perspectives of the eleven participants in my study, who discussed their ideals for design and delivery of inclusive education through conversations about challenges and benefits of inclusive education. There are three sections in this chapter: First, challenges of implementing inclusive education, second, perceptions of ideal inclusive education spaces, and third, an analysis of the challenges and construction of inclusive education spaces identified by participants, whereby I develop a model of the spaces required for inclusive education to occur. The chapter concludes with a discussion of this model and its relationship to wider literature and theory in the field of holistic approaches to inclusive and refugee education. 


\section{Challenges of implementing inclusive education}

Participants identified three key challenges which affected the design and delivery of inclusive education. Aligning with some of the research and literature identified in Chapters One and Two, participants described how labelling and perceptions of disability and refugee background people influenced government and NGO responses, how the lack of integrated services and funding meant that educational responses were often weaker and less inclusive than ideally they might be, and how organisations were siloed. I examine each of these issues in turn.

\section{Labelling and perceptions of disability}

The labelling of disability is contentious (Worth, 2008, 2013), and participants identified this as a major challenge when providing inclusive education for refugee background people with disabilities. Education provider participants in particular identified labelling as a challenge when implementing inclusive education for refugee background people with disabilities. As people who dealt with the implementation of inclusive education policies, the education providers were the only participants to comment directly on the challenges of implementing inclusive education, whereas people in government organisations generally created and developed such policies but didn't implement them.

All participants described how disclosure and labelling of disabilities were big issues and were linked to the amount of funding their organisations received. Participants who worked in non-governmental organisations and government organisations had a different level of understanding of refugee background people's experiences and the impact of disability upon education. Participants from nongovernment organisations generally perceived physical disabilities as 'easier' to deal with compared to mental disabilities, as the disabilities were often visible. These participants could have also been less familiar with mental illness and disability. NGO4 stated that they had

"worked with people who've had a disability because of conflict, and that makes it easier for people to realise, because they see someone who's come from war 
and the impact of war, and that's easier for teachers to say 'well obviously they've come from a dangerous place' and have the related physical scars."

Participants were also unaware of invisible disabilities unless they were disclosed by the refugee background student. Consequently, undisclosed and invisible (especially mental) disabilities meant that providers couldn't provide support to the refugee background students. This was emphasised by EP1, who said "because they don't disclose, we don't know who they are, so we can't provide support. I think that's really one of the key barriers".

Earlier comments on definitions of disability and inclusion may justify why participants were hesitant to place labels on students, as the definitions were quite broad and dependent on individual interpretation. As mentioned in Chapter Three, Worth discussed how the language of disability meant that some people did not choose to identify as disabled. She also discussed how some participants were able, or unable, to negotiate their identity while being labelled different and disabled (Worth, 2008, 2013). EP1's comment illustrates some of the contested nature of disclosure and labelling and the subsequent challenges for education workers. Providing students with the option of disclosing their disability increases the onus on education providers to provide inclusive education from the beginning of an individual student's journey, regardless of their background. EP1 also stated

"we need to be clear when we ask that information what it's for [...] we haven't come up with an ideal way of collecting the data, and we need to ask ourselves why we need to collect the data, and for what benefit, for whose benefit and for what purpose".

Her statement demonstrates the importance of informing refugee background people with disabilities why data needs to be collected. EP1 also stated that inclusivity is more than just what occurs in the classroom, and there is a link between disclosure of refugee background, labelling of a refugee background, and resourcing which could be provided to facilitate inclusive education spaces.

The issue of how and when to label or name 'disability' is a long-standing one, which was confirmed in this study and others preceding it (Connor, 2013; Crooks, 
Dorn, et al., 2008; Worth, 2008, 2013). Participants felt that unless refugee background people chose to disclose and accept their disability, and were willing to receive support, there was nothing they could do. EP1 stated that "the barrier that I perceive is from the refugee background community themselves, whether they are willing to identify themselves as refugees, but also disclose disability". EP1 also identified that a reason against disclosure was that "disability is regarded as a taboo subject in refugee communities [...] there's that whole stigma around disability” (EP1). This created a catch-22 situation for participants, as "unless the refugee background communities are willing to discuss the issue of disability and disclose that and work with the relevant community to address that, we can't actually include them" (EP1). EP1's statement also demonstrates how discourse and practices are linked. Some participants discussed ways of mitigating the negative effects of labels, and encouraging positive disclosure for refugee background people with disabilities. EP1 stated that

"from an institutional perspective we need to look at what the things we can do to minimise the barriers and maybe in terms of how we get them to do disclosure, in terms of bureaucracy and the administrative barrier we set up".

This quote emphasised that the issue of disclosure was one to be considered by both the students and the providers. EP1 further reiterated this view by saying "Institutions need to minimise barriers for students to access support. It kind of takes two to tango, there needs to be both forms occurring".

Disability could be perceived as a taboo subject or label within refugee background cultures. Labelling a person as refugee background and/or disabled can demonstrate the power that the labeller has over the labelled (Connor, 2013; Taylor \& Sidhu, 2012). While I didn't interview refugee background people in this research, participants discussed how disability was often viewed as holding a certain stigma. NGO5 said

"we have some families who look down on people with disabilities because they have come from a different cultural context. There are people who don't understand people who don't have physical disabilities, like invisible disabilities for example”. 
This statement demonstrates the significance of cultural norms and upbringing when understanding disability and how disability issues are complex and challenging for some groups. Therefore, special approaches are needed in order to educate people about the positive aspects of disability. EP1 also stated that

"the whole refugee label is quite... it has the same issues for people with disabilities or mental health issues [...] we want to connect them with the support we can provide for them. But again the disclosure is a big issue".

The example demonstrates that individual acceptance of a label, along with positive reinforcement by teachers and peers, can help to facilitate an inclusive education space which embraces a student's individuality.

In addition, attention needs to be paid to people's different perceptions of disability, as it could affect interactions and understanding. A lack of understanding and discussion surrounding disability could also affect people's knowledge of disability. NGO5 stated that they had

"heard some comments from families in relation to how they look at disabilities. One of the families said in relation to their [child] that medication causes autism, which is not medically proven".

However, it was important to acknowledge the "client's" views and "listen to what they were talking about and their feelings". Although NGO5 said that they listened to the clients, their overarching goal with to direct the families to a "more appropriate approach for thinking of things". This indicates that NGOs were responsible for altering people's perceptions of disability to one that was accepted by western society. This research links to wider discussions of power relations between disabled and abledbodied individuals, refugee background people and society (Connor, 2013). Language can affect whether a person chooses to define themselves in particular ways, and certain words and phrases can hold significant power (Worth, 2013).

\section{Limited funding for refugee background people with disabilities}

Participants from both Melbourne and New Zealand identified funding limitations as a major challenge when attempting to implement inclusive education 
spaces for refugee background people with disabilities. This was a significant focus for workers in NGOs in particular who appeared to be constantly in need of further funding. However, the discourse surrounding funding appeared to be stronger from Australian participants, than their New Zealand counterparts. The discussion around service funding occurred earlier in the interviews with Australian participants, as it was raised earlier in the conversation. It also appeared to shape their perception of refugees themselves. The effect of neoliberal reform was seen in participant discussions of aspects of educational policy, which connected to the literature (Kearney \& Kane, 2006; Nairn \& Higgins, 2011; Selvaraj, 2015).

EP3 and EP4 discussed how there were several stages to obtaining funding for students with disabilities. They also discussed how refugee background students (with or without disabilities) were all in the same classroom in their refugee-focused educational institution, but the situation could change when they moved into mainstream schools. Education providers also pointed out how service funding significantly affected their ability to provide inclusive education spaces. Limited resource funding also affected the amount of people who could be trained in accordance with inclusive education principles (Kearney \& Kane, 2006; Nairn \& Higgins, 2011; Singal, 2008).

While the EP participants were able to provide information about the practical implications of funding in classrooms, participants from NGOs and GOs provided information on the implications of funding on policy. Some participants identified a primarily economic approach to education, and the importance of funding in providing services to people with disabilities. GO1 mentioned the necessity of changing the funding system to benefit people with disabilities. He said "looking at the deficit of services that are currently available to people with disabilities a look at providing some funding to stop that would probably be an area that needs fixing ". GO1 also mentioned that a lack of funding was

"Certainly the criticism of the service providers when they were trying to find money to be able to start these classrooms with a high proportion of refugee background people with disabilities. I don't know why. It seems crazy that the government is not following up with support for these learners. You can't help 
them integrate into New Zealand society because the support system and the funding system's just not there. So it's totally illogical, and it's not necessarily a huge amount of money either".

This statement also illustrates the frustration of sector workers at the financial constraints placed upon their desires to offer inclusive education, and the negative effect these constraints had upon refugee background people with disabilities. There was a recognition of the incongruity of government resettlement goals with the policies and actions taken to integrate refugee background people into New Zealand society (McBrien, 2014). Participants also stated there were funding constraints when training staff about inclusive education principles. NGO2 stated that

"Unfortunately there are financial constraints but principally we make sure that staff are aware and know how to engage with people from all backgrounds, and know how to do it appropriately, and of course we hope to implement training and assist with that process in a more professional way."

Funding constraints even included communication with former refugees themselves, EP3 stated that

"Our biggest challenge is communication [with refugee background people]. Because you can't get interpreters for every language, and they're not available all the time, and it costs money. So not only do we have to provide special needs support, we have to provide interpreters which doubles the cost. And because communication is so important if you haven't got a language in common, you're going to delay the learning too".

NGO5 emphasised that it was important to discuss with refugee background people how the state and NGO provision of services worked financially. Doing so helped people to understand the limitations faced by service providers. NGO5 stated that

"Some people don't understand how the system works. And we have to tell them how it works. People don't seem to be open to change, sometimes it's really political, when the government changes, the service focus changes."

This statement indicates that there is occasionally tension between service providers and refugee background people on the basis of provision. Tension also exists between 
service providers and the government, as their funding is contingent on government grants (Marlowe et al., 2014; McBrien, 2014).

NGO participants in Australia and New Zealand also emphasised that changing government structures affected the amount of funding they received every three years. NGO5 stated that the proposed National Disability Insurance Scheme in Australia would change how their services are provided, as people with disabilities would be able to choose which services they would spend with their funding. He stated "in the new funding scheme they can continue to purchase our services or use the money otherwise. There are a number of political factors". The implementation of the scheme indicates the changing relationship between service providers and the government. NGO5 also explained that their service was "subject to regular audits and we have to make sure that all clients are updated and we are open to feedback, and part of my role is to report these complaints and feedback". NGO5's statements emphasised that their nongovernmental organisation was a service, and therefore had client-provider relationships. This undermined the participatory nature of inclusive education as discussed in Slee (2001) as the service provider always had "to be clear about what we can and cannot do". NGO5 commented that doing so was "challenging and requires a certain level of understanding”.

The emphasis on funding in order to provide services affected some NGO participants' perceptions of refugee background people, and a couple used economic terms to describe their relationship with refugee background people. Terms such as 'client' were used to describe the refugee background person, and service limitations were often described in terms of the amount of funding received. NGO3 stated that she worked with "clients with complex needs" as part of their role to "work with them out in the community". Similarly, NGO1 stated that she did "really intensive goalorientated work with my clients". There was an assumption that clients needed to be improved upon. NGO participants also quantified client experiences, and referred to client assessments for disability. These assessments were discussed in monetary terms. NGO participants often developed relationships with external service providers in order to obtain cheaper services for their clients. When describing a positive experience working with refugee background people with disabilities, NGO1 said "well I did a 
neuropsychology assessment on a client which turned out to be a lot cheaper". She stated that "she [the psychologist] provides a special rate for working with refugees... she usually gives a $\$ 500$ discount". While this described a generous practice, this language could dehumanise refugee background people by reducing their status to items in an economic system, instead of individuals with differing and multiple needs and strengths (Marlowe \& Elliott, 2014; McBrien, 2014).

One way of mitigating the effect of limited funding that participants identified was for NGO services to work together. The connections that NGO participants developed with external service providers were also important in ensuring that refugee background people received appropriate support. NGO1 said of her relationship with the psychologist

"she helped me, and I've held on to that connection because she's really... not only does she provide [her services] at a cheaper rate, which is great, but I know that she understands the issues and she gets it [...] she's not racist, which here [in Australia] amounts to a lot".

NGO1's statement also demonstrated the different discourses between Australian and New Zealand non-government organisation participants. For this group of participants, racism, and its effects on funding and service provision was a more dominant feature of Australian participants' discussions than New Zealand ones (Kirk \& Cassity, 2007).

\section{Working in silos}

Some participants identified fractured communication systems of organisations working in silos as the third major challenge affecting the provision of inclusive education spaces. Such silos prevented communication across different areas within the refugee resettlement sector. Both participants from government organisations, however; maintained that this wasn't a problem. GO2 stated that he maintained communication with other government agencies, and emphasised the importance of communicating with frontline agencies to ensure that funding targets were met. He said

"we've got engagement with the frontline agencies themselves, to make sure that we're sharing information. If we're putting money into something, or thinking about funding something, that we know what others are doing." 
GO1 echoed this rhetoric, saying "it's definitely driven by dollars and all the little things [...] it's all about the end result".

One positive example of communication between organisations was that participants from education provider organisations worked closely with the government to access funding for refugee background people to participate in education programs (Marlowe et al., 2014; McBrien, 2014). EP1 stated that their organisation "work closely with the government around [funding] and other institutions who may also be offering pathways for education for refugee background people”. EP4 also said "we'd like to know how they get on, what school they end up at".

However, EP4's remark was followed by EP3 saying “there's not a lot of feedback, there's no mechanism. And everyone's busy". Each organisation working with refugee background people appeared to concentrate on the goals of their own organisation, which could negatively affect communication and collaboration with other organisations in the sector. NGO3 emphasised this, saying "I think that the services are really fragmented, there's a lot of little things that've been set up for little bits of money. It's really hard to find out who does what". These comments demonstrates that there are direct links between communication, funding, and education (McBrien, 2014; Nairn \& Higgins, 2011). When working with refugee background people (with or without a disability), communication is important as it directly affects educational outcomes and services (McBrien, 2014; Mortensen, Latimer, \& Yusuf, 2014; O’Rourke, 2011).

\section{Participant perceptions on the construction of inclusive education spaces}

So how do people working with refugee background people create inclusive educational spaces? This section considers the design and delivery of three key aspects of inclusive education spaces that participants identified as providing more inclusive forms of education. These three aspects include physical, social and affective, and cultural and intellectual processes. This discussion concludes with the inclusive education model I have developed from discussions with these participants, which answers research question three. 


\section{The physical space}

The physical environment is significant when creating inclusive education spaces for refugee background people with disabilities. All participants identified that there was room for improvement in the physical spaces. Educational provider participants who had the most experience in this area commented that the Western-style classroom spaces were not necessarily conducive to learning, particularly for students of different cultures (Humpage, 2009). Two service providers (NGO2 and NGO3) and all education providers suggested that the removal of desks and chairs in favour of more comfortable seating arrangements would improve the classroom dynamic and overall inclusivity.

Participants also acknowledged that an ideal inclusive education space "doesn't generally have to be physical" (EP1). EP1 expanded upon her initial statement, saying

“ideally there's an indication that it's physical, but it should be a community, where everyone who walks in knows that they're valued; they are respected [...] there is no shame or fear of disclosing their background".

The idea of an inclusive education space was "broader than geographical location" (EP1). EP1 also stated that "when you talk about inclusive education it should be manifested in every level of the institution [...] it's a little conceptual". This statement emphasised the idea that inclusive education is the combination of a variety of spaces which move beyond material notions of space, linked to relational notions of space (Massey, 2005) and space as an affective or emotional site (Kenway \& Youdell, 2011).

Participants mentioned the importance of taking personal experience into account when creating inclusive education spaces. Participants recognised that spaces needed to be designed so that people of differing physical needs could all access the space equally. People may have the same experience of an education space, but it is the individual's emotional association with the space which can alter perception. Creating inclusive education spaces specifically for refugee background people with disabilities, such as separate English language courses as suggested by some participants, could be 
beneficial to students' sense of emotional connection and belonging (Kenway \& Fahey, 2011; Kenway \& Youdell, 2011; Worth, 2013).

There was recognition that an inclusive education space had defined boundaries; ideally students would feel comfortable upon entering due to the positive physical and emotive effects of the space. The idea of boundaries was emphasised by participants stating the values associated with inclusive education spaces. EP1 stated that in an ideal inclusive education space there was

“mutual respect, there's no discrimination, there's no stereotyping of the individual by the community, there's a real genuine open mindedness and authentic exchange between people who value difference".

This statement links to earlier discussions of democracy in inclusive education (Freire, 1993; Michailakis \& Reich, 2009; Slee, 2001). EP1, however, questioned the idea of creating certain spaces of inclusive education, saying "I sometimes wonder if having a dedicated space for that purpose is the best way to go, because it could end up assigning inclusive education to a particular space”.

Her statement suggests that inclusive education could be present in multiple spaces, and not always confined to a certain space within an institution which could instead promote exclusion. EP1's quote touches on debates within geographies of education and disability on the positives and negatives of segregated spaces for people with disabilities (Holt, 2003, 2010; Worth, 2008, 2013). A segregated space could provide a safe, inclusive space for refugee background people with disabilities, however, segregated spaces can also emphasise exclusion, as people who enter the space can be defined as 'different' in both a positive and negative sense. This can undermine the idea of an inclusive space, as the space essentially operates on the basis of exclusion (Holt, 2010; Worth, 2013; Ypinazar \& Pagliano, 2004). In order to ensure that a space is inclusive, everyone must be included. This echoes Thiem's (2009) idea that education spaces are a reflection of wider society- in order for society to be inclusive for everyone, everyone needs to be included in education spaces. 
Inclusive education spaces that were identified by participants were not necessarily traditional learning environments. Participants included a range of spaces which fostered different aspects of inclusive education. EP1 stated that a positive inclusive education space in their institution was found "within our student support unit. We have a space that we cater for Pasifika students, Chinese students and Maori students for their learning”. They also stated that they "don't as of yet have a specific dedicated space for refugee background students, partly because refugee background students are not yet an equity group". As refugee background students were not considered an equity group by the government of New Zealand and therefore not receiving additional funding, there was little incentive to create a dedicated space specifically for their inclusion. However, EP1 stated that they were "working towards a culture space, where our students from diverse communities can use that space for cultural interaction, for diverse support". This discussion on funding the development of inclusive education spaces relates to the emphasis placed on citizens to be selfsufficient, fueling neoliberal economies. In doing so, undetermined equity groups (such as refugee background people) miss out on funding opportunities which could benefit their schooling (Freire, 1993; Nairn \& Higgins, 2011).

\section{The relational space}

The relational space is defined as a combination of social and emotional spaces discussed within inclusive education, and is the space where students interact with peers and teachers. Social interactions and affective responses can influence whether a student feels included in an education space. The classroom's spatial arrangement for disabled and refugee background students can affect a student's social interaction outside of the education space. As an inclusive education space involves the combination of relational, pedagogical and physical spaces, students with little social interaction are unlikely to feel included if they are excluded from their peers (Worth, 2013). Additionally, little social interaction can lead to feelings of isolation and insecurity, which negatively affects student's emotional perceptions of the education space.

Developing a sense of place and belonging is important in a refugee background person's transition into New Zealand and Australian culture, and this development occurs in relational spaces (Marlowe \& Elliott, 2014; Massey, 2005). EP3 and EP4 
stated that developing a person's sense of belonging was an important aspect of inclusive education. The importance of making space for emotions of belonging, safety and attachment in inclusive education was recognised by education providers (Evans et al., 2009). EP3 said:

\section{"[...] People do go to a lot of trouble to create the spaces required for different} needs. But I think the most important space is the emotional space, that's the space we've got to be very careful about and make sure everyone feels safe. We've got to watch the language, watch the behavioural language [...] as teachers [both] professionally and in a caring way so everyone is valued. You can 't put down one person because they've put down another person. We're teaching a learning environment, it's always about learning. We do have a very good emotional space. It's one of the things refugees often comment on".

The combination of physical and relational spaces can directly influence one's experiences of inclusion, as participants emphasised. Experiencing inclusive spaces, physically and emotionally, can also help students develop a sense of place (Kenway \& Youdell, 2011). EP1 commented that "we do have a generic social learning space where everybody can be there [...] it's not tailored specifically for anyone from a specific community. It kind of provides an open forum for everyone to interact regardless of where they come from".

Power can also facilitate belonging. Refugee background people and people with disabilities are widely perceived as the 'other' by society. Edward Said and Paulo Freire state that the 'other' needs to have power in order to achieve in education (Freire, 1993; Said, 1978). Freire also argued that education should be used as a method of freedom, allowing the other to move beyond constrictive boundaries (Freire, 1993).

Applying ethics of care into the classroom helped to foster inclusive education spaces and directly relates to the application of language and power in education. EP3 and EP4 discussed how they could demonstrate "models of care" (EP4) to refugee background students with disabilities. These models of care related to their pedagogies that were practised in the classroom. EP1 also stated that "looking at other forms of pedagogy which encourages greater forms of learning " was important when assessing 
inclusive education. Models of care can also help to facilitate student belonging in a classroom. Occasionally the models of care imposed upon students with disabilities by staff could affect social interaction within a school (Collins \& Coleman, 2008; Worth, 2013).

Teachers also identified themselves as being 'role models' for appropriate behaviour and encouraging acceptance of differences. They emphasised the importance of verbal and visual language in order to create an appropriate intellectual and emotional space. EP3 said

"they're [students] picking up a whole lot of other cues from you because they're not understanding everything that's coming out of your month [...] picking up on these sorts of things become[s] really important and you can't hide them if you've got some little prejudice".

Her comment shows that teachers are often the first points of contact refugee background people have when arriving in New Zealand. Consequently, teachers need to be aware of their presentation in order to facilitate an inclusive learning space.

Participants also often mentioned the word 'safe' when describing ideal inclusive education spaces. EP1 stated that

"ideally if we had a space where students can feel safe when they come into that space, to meet with other students, and to meet with students from their own community, a diverse community, where they don't feel that they have to hide their identity [...] safe to disclose who they are".

This statement suggests that identity and safety within a space are intertwined concepts. Collins and Coleman (2008) state that schools are the most influential aspect of young people's lives as students are monitored by teachers and educational 'experts', and subjected to disciplinary measures. Students are a part of underlying power structures within the school, and are generally perceived to be under the control of staff. Although safe spaces could be beneficial for refugee background students with disabilities, they could also create tension between staff and students if staff wanted more ownership and control of the space (Humpage, 2009). 
EP3 and EP4 also discussed the individual safety of refugee background people in education. They described a situation where a transgender person "spent a lot of time here in our staffroom. That was where she felt safe, could have a cup of tea". This statement suggests that safety sometimes cannot be found in a seemingly inclusive classroom, due to other people's perceptions - including those of refugee background people. EP4 said "sometimes those challenges for us are greater for others aren't they, in terms of acceptance and inclusion”.

Relational spaces are mutually constructed through social interactions and relationships (Lefebvre, 1991; Massey, 2005; Thiem, 2009). These social relationships that occur within a relational space can help to create a place (such as a school) that is both emotional and meaningful for students. Consequently, these spaces can become 'safe' for students, which benefits their learning (Worth, 2013). The act of entering safe spaces, however, can be a form of disclosure which could negatively affect social interactions. People would recognise that the student was entering the space because of their needs, and the student would be associated with disability. Therefore, care needs to be used when discussing 'safety', as separate inclusive education spaces may not encourage safe spaces in all areas of education (Humpage, 2009; Worth, 2013)

\section{The pedagogical space}

All participants identified inclusive education as being broader than traditional Western definitions of education. Inclusive education for refugee background people incorporated learning about culture and identity, as well as developing English language, literacy and numeracy skills. Participants recognised that there were different pedagogies for teaching refugee background students. Matthews also states that "refugee movements are an effect of colonial legacies and contemporary power struggles" (2008, p. 34), which emphasises the importance of recognising the interplay between neoliberalism and colonialism in education. Different pedagogies are essential when teaching cross-culturally, as people learn in different ways. The dominant neocolonial discourse which permeates most current teaching practices was not seen by participants as conducive to inclusive education. This neo-colonial discourse privileges 
western methodologies and emphasises western cultural dominance over non-western cultures. This idea was identified when EP3 said

"We do have that way of discussing things that is participatory, all the ideas without hostilities. When I first started this wasn't like that, it was 'this is the NZ culture, fit in or go home'. And that was very much part of what New Zealand was, it was done to Màori people in schools, and that wasn't long ago. And there's no participation of language, no place”.

EP3's comment demonstrates the importance of changing pedagogies in order to facilitate inclusive education. Her comment above also demonstrates how colonial discourses can negatively affect education for a myriad of cultures, and remove participation (Taylor \& Sidhu, 2012; Zembylas, 2013).

Power is also an important aspect to consider when evaluating participatory pedagogies of inclusive education. EP3 and EP4 emphasised the importance of asking refugee background people how they do things in their home cultures, and sharing cultural information. EP4 stated that "there's a methodology that you can employ that assumes power and there are methodologies that don't'. Discussing aspects of culture with refugee background people, rather than speaking at them demonstrates a shift in power dynamics. The traditional linear power structure between teacher and student is melded into a more circular framework; one where the teacher and student learn from each other. EP4 stated that there was a fine balance which existed; "giving people ways that they can behave that are culturally OK but also not going to exclude others in social environments where we find that generally unacceptable”. This example of balancing ideals demonstrates the multiplicities which exist when dealing with power and culture. The interaction between power and culture in education is messy, particularly when different cultures are involved (Taylor \& Sidhu, 2012; Zembylas, 2013).

In order to have a successful inclusive education space, teachers and students need to have an understanding of each other's learning strategies. Although education providers interviewed discussed the importance of being role models for students' behaviour in the classroom, literature suggests that they need to be mindful of stifling 
students' social interaction (Worth, 2013; Zembylas, 2011). EP3 and EP4 appeared to recognise this connection in their discussions of student interaction. They stated that there needed to be

"quite serious conversations and also giving people ways that they can behave that are culturally $O K$ but also not going to exclude others in social environments where we find that [behaviour] generally unacceptable”.

The social aspect of school is just as important as the academic aspect (Kearney \& Kane, 2006). One aspect cannot be privileged over the other, as social interaction and academic performance are intimately connected for students (Worth, 2013; Zembylas, 2011).

Having historical, social and cultural knowledge about refugee background people's experiences and pasts is necessary when providing inclusive education. Most participants either focused on aspects of inclusive education from the perspective of a refugee background person, or a person with a disability. One reason for this could be due to the individual that the participant felt more empathetic towards, or had more experience with one or the other. EP1's answer focused primarily on refugee background people and the issues that they could face which prevents access to education. She said that the reason for little education may be

“Because they're constantly on the run from country to country. So I think they bring a lot of baggage into the country and to be able to actually get into studying mode requires a lot of support, particularly from a social and economic point of view".

The "baggage" that refugee background people carry may be invisible, as well as visible. There are legacies associated with previous traumatic experiences of refugee background people. However, these legacies are often aligned with resilience strategies which can help refugee background students navigate the education system.

There were two different groups of refugee background people discussed in the interview with EP1. She said "there are those who have just entered the country but there are those who are second generation [...] so there's therefore that generation gap 
too". Therefore, it is important to be mindful that aspects needed for inclusive education may change depending on the individual's circumstance (Michailakis \& Reich, 2009).

Cultural knowledge and support of refugee background people was as a key aspect of inclusive education. Extra support needed to be provided to refugee background people entering the Western education system (Marlowe et al., 2014). EP1 stated that "culturally appropriate support to enable them to succeed and thrive and participate in this community" was necessary for refugee background people with disabilities. The combination of support and successful educational practices would be beneficial to refugee background students. She also said that the "social, emotional and financial" support was integral to educational success. Financial support was crucial to helping refugee background people stay in education, as "quite often people start to drop out as the financial pressure mounts".

These three pillars of support required for inclusive education form a holistic model. EP1 acknowledged that "you can't divorce [...] their personal life from their academic life, it's interlinked". She also stated that "students aren't going to do well if they have a whole lot of other personal issues that are not being dealt with". This statement emphasised the need to "integrate holistic support for our students and address the whole person rather than just compartmentalise their needs" (EP1).

Holistic support before and during a student's education experience is needed for inclusive education to be achieved. EP1 also stated that "to come into a Western education context they need to first of all navigate the language and understand how the Western education system works, which is very different to where they come from". Being mindful of previous experience and exposure to the education system is also crucial for inclusive education (Kearney \& Kane, 2006; Marlowe et al., 2014; Slee, 2006).

In addition, "having the academic language support is also quite crucial as part of that inclusive education" (EP1). Providing language support therefore could help mitigate the barriers presented by the Western education system, which in turn provides 
more inclusive education practices (Marlowe et al., 2014). EP1 then linked the Western education system to Western culture, and the challenges that this could present to refugee background students. She stated

"the non-Western culture is generally more holistic in the way they view things, non-linear. The Western culture is very linear, very dualistic so we can compartmentalise. But from a non-Western perspective life is very interrelated, it's encyclical [sic]".

This statement demonstrates that the differences between the Western and non-Western education systems could be a barrier to refugee background students participating in education.

The participants' frustrations with disclosure and labelling of disability, funding and silo practices (as stated earlier in the chapter) was evident in their discussions of pedagogical spaces. They recognised that change was still needed, and that actions towards change had to be bold, transformative and, at times, radical. As EP2 stated:

"I think just doing it is really needed. I think that my experience in pushing any kind of social change is that you just do it and bulldoze over people a little bit. People will adjust, they think it'll be impossible but they will do it [...] there's no 'should we include people?' it's this is happening, let's do it".

This statement suggested that there was a need for practitioners to be 'bolshie' and implement inclusive education practices themselves, rather than waiting for bureaucratic changes to occur. In order for inclusive education to be successful, it needs to start with individual changes. Individual change will lead to collective action. 


\section{Towards a holistic, integrated model of inclusive education}

Figure 5.1: The three intersecting spaces required for inclusive education

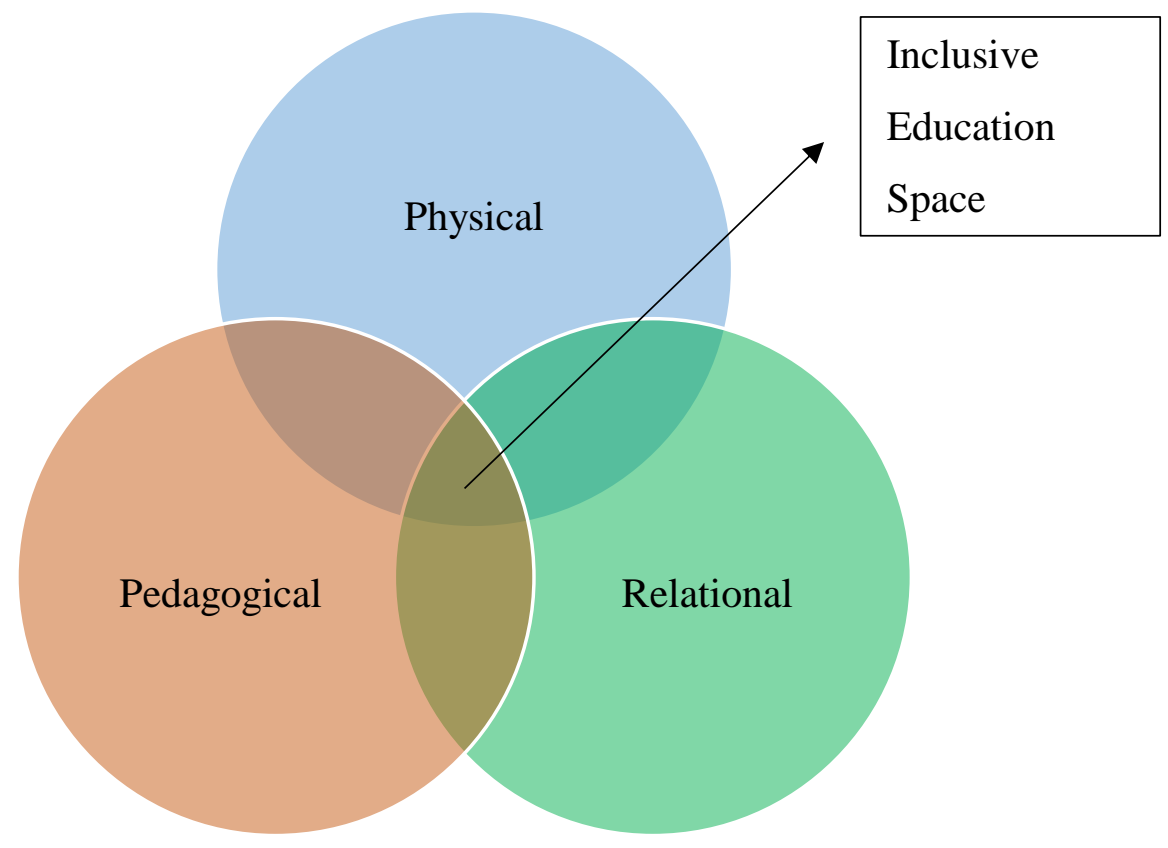

In the final section of this chapter, I suggest a way of integrating the physical, relational and pedagogical spaces toward a more holistic model of inclusive education space, based on the analysis of participants' interviews and applying theoretical ideas from spatial and feminist approaches outlined earlier. This model is underpinned by ecological models of inclusion and disability and applies specifically to refugee background students with disabilities. The participants' discussions (who can be regarded to some extent as 'experts') in policy and practice fields in this area contribute to the development of an intersecting, three-fold model of inclusive education which includes the intersection of physical, relational and pedagogical spaces with one another. One limitation of figure 5.1 is that it focuses on students with disabilities, and does not consider some of the limitations which participants previously discussed (i.e. safe spaces for transgender students). Figure 5.1 demonstrates the three key spatial components that participants identified for inclusive education and inclusion to be able to occur: the relational space, the pedagogical space, and the physical space. Henri Lefebvre (1991), Doreen Massey (2005), and Claudia Thiem's (2009) theoretical approaches to social and relational space also illuminate aspects of the data, and have helped to identify the three key dimensions of my inclusive education model. 
The physical space links to Lefebvre's interpretation of perceived space. The perceived space is considered to be how physical objects are placed in space and nature (Lefebvre, 1991; Stanek, 2011). The physical space is important when considering how spaces can be exclusionary for refugee background people with disabilities. The debate between segregated and mainstream schooling (as discussed in Chapter Four) can raise issues of exclusion based on physical spaces. This debate echoes Thiem's (2009) idea of education spaces as a wider reflection of society. However, some participants suggested that segregated schooling can also provide inclusive education spaces as they can specifically cater to students with disabilities. They also identified that students may have different physical needs which needed to be catered for in order to have an inclusive education space. As participants discussed earlier, considering the physical space is also necessary when determining how a classroom could become an inclusive education space.

The pedagogical space relates to Lefebvre's (1991) idea of the conceived space, and the theoretical representations of space. It also relates to Lefebvre's idea of the significance of power in relation to space, which was discussed in Chapter Two. The education provider participants emphasised that sharing power between teachers and students was essential to successful classroom pedagogies. The concept of power in the conceived and pedagogical spaces is especially important when considering the impact of colonial teaching methods. The Western education system can prevent students from different cultures from successfully participating in classrooms, and remove their power (Sidhu \& Taylor, 2007; Taylor \& Sidhu, 2012). As people learn in different ways, it is important to have knowledge of different pedagogies which could be applied in the classroom.

Knowledge of power in the pedagogical space can influence teachers to develop positive physical and relational spaces, which in turn creates a successful inclusive education space (Lefebvre, 1991). As stated earlier in the chapter, the majority of participants focused on pedagogies which related to either refugee background people or people with disabilities. Having knowledge of the social, financial and cultural support that refugee background students require could further develop education 
practitioner pedagogies. Doing so requires knowledge of how education affects society, and vice versa (Thiem, 2009). Pedagogies do not exist in a vacuum, they affect aspects of students' lives outside of the classroom. Consequently, pedagogical spaces need to be considered in relation to the physical and relational spaces, as all spaces mutually cooperate to form an overarching inclusive education space (Massey, 2005; Thiem, 2009).

The relational space in Figure 5.1 links social and emotional spaces, and emulates Lefebvre's (1991) lived space, and Massey's (2005) ideas of space as related and socially constructed. The relational space is necessary when developing an inclusive education space as it fosters a sense of belonging, and helps students to develop a sense of place (Kenway \& Youdell, 2011). Participants discussed how relational spaces could also develop students' identities through social relationships and 'safe' spaces of inclusion. Having spaces of representation through social relationships is necessary for inclusive education spaces to develop (Lefebvre, 1991; Stanek, 2011).

The relational space is where social interaction takes place, and is constantly altering depending on individual relationships to each other and the environment (Massey, 2005). Space cannot be viewed as a silo, as there are always related processes occurring on and beneath the surface (Massey, 2005). Therefore, the relational space is important for inclusive education spaces as it ensures that the stakeholders involved are not working in silos. Implementing the inclusive education model depicted in Figure 5.1 could mitigate the challenges that participants faced working in silos. The relational space also demonstrates how power could be shared between students and teachers in inclusive education, and that working together could reduce the silo that the 'other' label creates (Freire, 1993; Said, 1978).

The intersecting space between the pedagogical, physical and relational spaces in Figure 5.1 demonstrates an ecological, holistic system. The ecological model is established by time, space and context (Kenway \& Fahey, 2011; Kenway \& Youdell, 2011). The inclusive education model relates to the ecological model of disability, and emphasises a holistic approach to disability (Ebersold \& Evans, 2003). The inclusive 
education model could also help participants to perceive disability and 'otherness' in relation to the ecological model (Ebersold \& Evans, 2003). The education provider participants' experiences of disability enabled them to develop pedagogies which included different aspects of disability. Their knowledge of pedagogical, conceived spaces helped develop physical, perceived spaces which were inclusive for people with disabilities. In turn, students found belonging in the relational, lived space based on participant interactions in the physical and pedagogical spaces. The interrelationships and unity that exists between the pedagogical, physical and relational spaces, and the application of the ecological model of disability can benefit interactions between teachers and students.

An ecological model of disability also incorporates different elements (objective and subjective) which are needed in order to make a space available for individuals with disabilities (Ebersold \& Evans, 2003). The ecological model also states that there are two elements of disability: the individual and the society. Both the individual and society need to work together in order to achieve full participation for people with disabilities. Society and the individual working together is also expressed in Figure 5.1. The merging of the circles suggests relationships between students and teachers in different spaces must occur in order to develop a truly inclusive education space. The physical space could represent collective society, as society generally creates the physical spaces for people with disabilities. The relational space could represent the individual, and the individual's thoughts and feelings related to a space. Incorporating both of these sections creates a space where the individual and the collective can coexist, making a space inclusive for people with disabilities. The development of Figure 5.1 in relation to the ecological model demonstrates that there are links between participants' definitions of access to inclusive education spaces (as discussed earlier in the chapter), and the implementation of inclusive education spaces. The ecological model of disability suggests that society and the individual both contribute to disability. The inclusive education model takes into account the societal and individual aspects of individuals, much like the ecological model of disability. 


\section{Summary}

The three main challenges which affected the design and delivery of inclusive education; labelling of disability, limited funding and siloed organisations, were all similarly identified across the three participant groups. However, as with Chapter Four, participants who directly worked with refugee background people were able to identify more challenges associated with their work.

All participants stated that labelling disability was a contentious issue, and one that provided challenges when delivering inclusive education (Worth, 2008, 2013). The participants from non-government and government organisations had a different level of understanding of the challenges facing refugee background students with disabilities in education, perhaps due to their indirect experiences. In contrast, the education provider participants had a more nuanced idea of how labelling affected the provision of inclusive education. This was similar to Chapter Four, where the education provider participants generally had more perceptive answers surrounding disability. Nongovernment organisation participants and education provider participants both discussed how disability could be perceived as taboo in refugee background cultures. This related to wider discussions in the literature on how labelling people demonstrates power over them (Connor, 2013). People can have different perceptions about what it means to be disabled or from a refugee background. Therefore, it is important to recognise the power of language, and to accept people's own definitions of themselves (Connor, 2013; Worth, 2008).

The education provider and non-government organisation participants had direct experience of the implications of limited funding upon service provision. Both participant groups expressed frustration at financial constraints restricting their organisation's abilities to work with refugee background people with disabilities. The financial limitations of organisations generally linked to government provisions (Kearney \& Kane, 2006; Nairn \& Higgins, 2011). Limited funding also affected communication between different participant groups. Participants expressed that there was not enough staff or time to maintain communication with other agencies, while working within their own organisations. 
The challenges identified in this chapter led to the development of an inclusive education model, based on participant interpretations of their ideal inclusive education spaces. In order for an ideal inclusive education space to form, participants identified that there needed to be physical, relational and pedagogical aspects of space. The education provider participants again had the most nuanced views regarding ideal inclusive education spaces, based on their direct experiences in education.

The intersection between physical, relational and pedagogical spaces leads to an ideal inclusive education space, depicted in Figure 5.1. I drew on spatial theories from Thiem (2009), Massey (2005) and Lefebvre (1991) to explain the importance of interrelated spaces in the context of inclusive education. I also used participant comments about ideal inclusive education spaces to emphasise the need for the model. Finally, the inclusive education model relates to the ecological model of disability (Ebersold \& Evans, 2003) as it shows a holistic integration of different spaces. The individual and societal interactions that occur within the pedagogical, physical and relational spaces ultimately create a holistic inclusive education space for refugee background people with disabilities. 


\section{Chapter Six: Concluding remarks and}

\section{recommendations for future research and practice}

\section{Chapter overview}

The final chapter of my thesis addresses the key conclusions from my three research questions, and how these conclusions position my thesis within current literature. In particular, I discuss how my results and discussion merge with the literature and theories presented in earlier chapters. I consider the wider implications of my research for the refugee resettlement sector and research in Geography. I also discuss what future research could be done.

\section{Overall conclusions}

In Chapter Four, I examined my results in relation to research question one what are participants' definitions and interpretations of inclusion, disability and inclusive education? All participants paused before providing definitions, and the majority of participants were unsure of their answers. This linked to current literature on the contentious nature of disability (Gable, 2014; Šiška \& Habib, 2012). All participants' definitions of disability and inclusion indirectly drew upon the social and medical models of disability. Participant definitions of which linked to the medical model emphasised that there was a hierarchy between disabled and able-bodied people, which affected their relationships with disabled people (Dear et al., 1997; Gleeson 1964, 1999). In contrast, the participant definitions which emulated the social model of disability linked disability to culture (Hassanein, 2015; Šiška \& Habib, 2012).

Participants' occupational status and experiences with disability and inclusion shaped their responses and perceptions. The education provider participants generally had the most direct experiences with disability and inclusion which led to more nuanced understandings and definitions than the other groups. Their definitions of disability and inclusion linked to the social and ecological models of disability (Ebersold \& Evans, 2003). In contrast, the non-government and government participants had a limited, indirect understanding of disability and inclusion at times. Their definitions were more 
functional, and reflected the nature of the participants' employment as service professionals.

There were three participants who related their definitions of inclusion, disability and inclusive education to personal experiences with disability through family and friends. NGO1, EP2, and EP4 were the three participants who explicitly stated links between definitions and disability in their personal lives. Their experiences with disability had clearly helped to formulate their understanding and perception of disability, inclusion and inclusive education. This linked to discussions within literature on the importance of personal experiences in understanding disability and inclusion (Dyck, 2000; Goodley \& Runswick Cole, 2014).

Participant definitions of inclusive education echoed aspects of Curcic et al's (2011) definition. As with the definitions of inclusion and disability, participants were hesitant when providing a definition for inclusive education. They echoed statements within literature that inclusive education should provide refugee background students with disabilities with choice in education (Hassanein, 2015; Kearney \& Kane, 2006). Although there were some differences in definitions, all participants found that inclusive education should be accessible for everyone (Curcic et al., 2011).

From the definitions, of inclusive education, a discussion arose on the differences between specialist facilities for students with disabilities versus mainstream schooling. This discussion linked to literature which emphasised how inclusive education developed from special education. There are still debates whether inclusive education should emulate aspects of special education, or branch away in literature (Kearney \& Kane, 2006; Slee, 2001). These debates were also demonstrated in participant discussions. EP2 particularly discussed her negative views regarding segregation of disabled students in schools, but acknowledged that some disability communities (such as the Deaf community) benefit from segregation measures. Both education systems have positive and negative aspects, and it is up to the individual to determine what system works best for them. 
The definitions and perceptions of disability, inclusion and inclusive education discussed in Chapter Four led to wider discussions of inclusive education spaces in Chapter Five. In Chapter Five I discussed my results in relation to research question two - 'what are their [participants'] perceptions of the challenges of implementing inclusive education spaces for refugee background people with disabilities, and how do they perceive ideal inclusive education spaces?' This question led to the development of an inclusive education model (as shown in Figure 5.1), which answered research question three, 'how could participants' ideal inclusive education spaces help create a more holistic model of inclusive education for refugee background people with disabilities?'

The challenges to implementing inclusive education that participants discussed highlighted three key areas: labelling and perceptions of disability, funding for services, and communication between organisations. Participants identified labelling as an issue, as some refugee background people chose not to identify as having a disability. In addition, disability can be a taboo label in some cultures where refugee background people come from (Connor, 2013; Crooks, Dorn et al., 2008). Refugee background people may also not wish to have the refugee label, as it can be associated with past trauma and inflict upon the idea of new beginnings. Participants felt that labelling and disclosure was an issue as although it was up to the individual, some services required disclosure of a refugee background or a disability in order to receive funding. Some participants also did not know which services to provide for some disabilities until they were told. Consequently, labelling and disclosure could help to make service delivery of inclusive education more effective (Taylor \& Sidhu, 2012). Overall, labelling was perceived to be a contentious issue, and one that was not easily remedied.

Service funding for refugee background people with disabilities was another major challenge identified by participants. Limited service funding affected the quality of inclusive education provided, and the ability of teachers to provide for individual needs in the classroom. Again, the education provider participants had direct experience of how limited funding affected classroom teaching practices. The non-governmental organisations also found funding difficult to obtain, as they relied on government contracts to continue providing their services, and spoke of creating alliances with other 
organisations in order to obtain cheaper services for refugee background people with disabilities. Non-government organisation and government participants also used economic terms to define refugee background people with disabilities also highlighted the importance of funding for these organisations, and the fact that they were providing a service to customers. The limited funding for services could be attributed to current neoliberal government funding models, and the reduction of funds towards education in favour of increasing employment opportunities. Ironically, studies show that education improves employment opportunities (Marlowe et al., 2014; McBrien, 2014; Nairn \& Higgins, 2011).

The last major challenge identified by participants was communication between different organisations in the sector. The majority of participants in non-government organisations and education providers felt that they were working in silos, and were often too busy to collaborate and communicate with each other. There was an underlying rhetoric within interviews that communication with government organisations improved chances of obtaining funding. The education provider participants stated that they worked closely with the government in order to receive funding, which indicated that the government had a strong influence on inclusive education practices. This linked to wider discussions in literature on the importance of communication between services for refugee background people (Marlowe et al., 2014; McBrien, 2014).

Participants emphasised that there were three key spaces required for inclusive education: the physical space, the relational space, and the pedagogical space. The physical space. The participants found that the physical space associated with inclusive education didn't necessarily involve the traditional 'table and chairs' model, and that refugee background students with disabilities may feel uncomfortable in traditional education settings. They emphasised the importance of a student feeling comfortable in a physical space, which related to further ideas in the literature surrounding belonging and emotional connections to space (Kenway \& Fahey, 2011; Kenway \& Youdell, 2011). Lastly, participants said that all students should feel physically included in a space, and not segregated based on disability or culture. Segregation was perceived as 
exclusionary, and negated the idea that inclusive education spaces should be reflective of wider societal inclusion (Thiem, 2009; Worth, 2013).

The relational space was perceived as a combination of social and emotional spaces necessary for inclusive education (Massey, 2005). Social and emotional spaces are necessary for facilitating belonging and developing a sense of place within education spaces (Kenway \& Youdell, 2011). The relational space also linked to ideas surrounding safety, and ensuring positive interactions between students and teachers. Participants recognised that students were subject to underlying power structures in education systems, which could affect their interpretations of safe spaces where they belonged (Collins \& Coleman, 2008).

When discussing pedagogical spaces, participants found that the dominance of Western pedagogy in education can reinforce colonial attitudes in schools. It is important to recognise that the majority of refugee background students do not come from Western education systems. In order to effectively implement inclusive education spaces, the different needs of refugee background students need to be recognised (Matthews, 2008). These ideas make the study of refugee background students with disabilities even more important. The studies highlight how refugee background students' experiences of education can be seen as part of a wider pattern of societal attitudes and actions toward marginalised groups.

The physical, relational and pedagogical spaces all connected to each other to form Figure 5.1, with inclusive education spaces at the centre of the connection. This model formed as a response to participant discussions and current literature on inclusive education spaces. I based the model upon Lefebvre's (1991) interpretation of space as perceived (the physical space), conceived (the pedagogical space) and lived (the relational space). I also used Massey (2005) and Thiem's (2009) spatial theories to emphasise the importance of different spaces to form a holistic model of inclusive education. 
The ideal inclusive education space model (Figure 5.1) also related to the ecological model of disability that was described in Chapter Two. The ecological model uses holistic aspects of the individual and society to describe disability (Ebersold \& Evans, 2003). Similarly, the inclusive education model used aspects of the individual and society in relation to the three spaces present. The inclusive education model moved towards a more holistic interpretation of disability and inclusion in its alignment with the ecological model of disability.

\section{Recommendations}

While this thesis represents a very small group of people involved in refugee education and support, there are a number of implications that are worth considering for policy and practice, and recommendations for further research. One of the unexpected aspects of my research was how much more nuanced and perceptive educational providers were when it came to discussing inclusive educational spaces for refugee background students than the other participants (NGO and GO participants). The research shows that education providers that were interviewed have a strong idea of how inclusive education spaces can be effective in classrooms, as they have direct experience and knowledge of applicable pedagogies. However, the educational providers that I interviewed were a small sample, and may not reflect the views of the overall educational sector if I had interviewed a wider range of people. In contrast, government and non-government providers interviewed may have more indirect knowledge of inclusive education spaces, as it is not something they deal with every day. Education providers are the ones who implement government policy, and government policy is the backbone of the New Zealand education system. Therefore, involving education providers in policy discussions is vital to creating effective practice. I also recommend that more research is undertaken with education providers when developing specific inclusive education policies. My thesis shows that it is important to have open communication and collaboration between different stakeholders when implementing inclusive education spaces for refugee background people with disabilities.

Another policy recommendation is to have clear-cut definitions of inclusion, disability and inclusive education in policy which can easily translate into practice. 
However, Gable (2014) stated that having a singular definition of disability could undermine the complex nature of the concept. Taking this into account, further discussions about disability and inclusion in all aspects of New Zealand society could encourage deeper and more inclusive understandings of disability. This is important as the definitions people hold can affect peoples' perceptions of inclusion and disability and policies related to these. In order for people to view refugee background people and/or people with disabilities in a positive light, there needs to be more education around perceptions of disability and inclusion.

\section{Further research}

Future research could expand in greater depth upon these groups, such as educators involved in various stages of education. Further investigations into the level of resources schools have for refugee background students would also be worth investigating. The research could also expand in scope by interviewing a wider sample of participants for future research, and particularly involving more government participants, as there were only two in my study, and a more in depth comparative study with Australia.

Involving refugee background people with disabilities themselves in future research would provide much needed perspectives in this area of research. Although participants in this study provided invaluable insights, they are not the direct benefactors of inclusive education in the classroom. Furthermore, I never went into New Zealand or Australian classes where refugee background students study. These additional forms of data collection could test the inclusive education model I propose in this thesis and look at a closer application of these ideas in the classroom.

Overall, my thesis contributes to the wider literature surrounding refugee background people and education by discussing education in relation to geography. The majority of previous studies focus on the psychological effect of education on refugee background people, and do not consider the importance of space in education. Incorporating space in discussions of inclusive education could lead to further research being undertaken on the spatial arrangement of classrooms. 


\section{References}

Altinkaya, J., \& Omundsen, H. (1999). "Birds in a Gilded Cage” Resettlement prospects for adult refugees in New Zealand. Social Policy Journal of New Zealand, (13), 112.

Australian Government. (2016a). Australia’s Humanitarian Programme 2016-17 Discussion Paper. Retrieved April 18, 2016, from http://www.border.gov.au/ReportsandPublications/Documents/discussionpapers/discussion-paper-humanitarian-programme_2016-17.pdf\#search=refugees

Australian Government. (2016b). Refugee visa (subclass 200). Retrieved April 15, 2016, from http://www.border.gov.au/Trav/Visa-1/200-

Barbour, R. S., \& Schostak, J. (2011). Interviewing and Focus Groups. In C. Lewin \& B. Somekh (Eds.), Theory and methods in social research (2nd edition, pp. 61-68). London: London SAGE Publications Ltd.

Berg, B. L. (2012). Qualitative research methods for the social sciences (H. Lune, Ed.) (8th ed..). Boston: Pearson.

Brandon, C., \& Bloom, A. (2014). Disability in New Zealand resettlement of refugees: The new hope for equity. In D. R. Mitchell \& V. Karr (Eds.), Crises, conflict and disability: ensuring equality (pp. 210-217). Abingdon, Oxon : Routledge, Taylor $\&$ Francis Group.

Cahill, C. (2007). Repositioning ethical commitments: Participatory action research as a relational praxis of social change. ACME, 6(3), 360-373.

Calder, W. (2014). "My story": valuing the personal narratives of refugee students in New Zealand schools. Knowledge Cultures, 2, 1-47.

Cassity, E., \& Gow, G. (2005). Making up for lost time: the experiences of southern Sudanese young refugees in high school. Youth Studies Australia, 24(3), 51-55.

Catlett, B. S., \& Beck, I. C. (2007). Participatory Action Research and the University Classroom. In N. G. Hofman \& H. Rosing (Eds.), Pedagogies of Praxis: CourseBased Action Research in the Social Sciences (1st ed., pp. 21-37). Bolton: Anker Publishing Company, Inc.

Chadderton, C., \& Torrance, H. (2011). Case Study. In C. Lewin \& B. Somekh (Eds.), 
Theory and methods in social research (2nd edition, pp. 53-59). London: SAGE Publications.

Changemakers Refugee Forum. (2011). An equitable education: Achieving equity status and measures to ensure equality for refugee background tertiary students in Aotearoa New Zealand. Wellington. Retrieved from http://crf.org.nz/sites/default/files/staff/An equitable education - Achieving equity status for refugee-background tertiary students in Aotearoa NZ_0.pdf

Changemakers Refugee Forum. (2012). The challenges faced accessing disability support services by Wellington's refugee-background communities.

Wellington.Retrieved from

http://crf.org.nz/sites/default/files/staff/Executive\%20Summary\%20$\% 20$ If $\% 20$ we $\% 20$ have $\% 20$ to $\% 20$ go $\% 20$ on $\% 20$ our $\% 20$ own, $\% 20$ so $\% 20$ be $\% 20$ it $\%$ 20-

$\% 20$ The $\% 20$ challenges $\% 20$ faced $\% 20$ accessing $\% 20$ disability $\% 20$ support $\% 20$ servi ces $\% 20$ by $\% 20$ Wellington's\%20refugee-background $\% 20$ communities.pdf

Changemakers Refugee Forum. (2016). Guidelines for research with former refugees in New Zealand The research issue Working with former refugees Values and attitudes Questions for planning research. Wellington. Retrieved from http://crf.org.nz/sites/default/files/staff/Guidelines for Research with Refugees in New Zealand.pdf

Chouinard, V. (1997). Making space for disabling differences: challenging ableist geographies. Environment and Planning D: Society and Space, 15(1), 127-135.

Chouinard, V. (2000). Disability, Geography and Ethics: Getting ethical: For inclusive and engaged geographies of disability. Philosophy \& Geography, 3(1), 70.

Collins, D., \& Coleman, T. (2008). Social Geographies of Education: Looking Within, and Beyond, School Boundaries. Geography Compass, 2, 281-299.

Connor, D. J. (2013). Who "Owns" Dis/ability? The Cultural Work of Critical Special Educators as Insider-Outsiders. Theory and Research in Social Education, 41(4), 494-513.

Cook, V. A., \& Hemming, P. J. (2011). Education spaces: embodied dimensions and dynamics. Social \& Cultural Geography, 12(1), 1-8. 
Corbett, J. (1999). Inclusive education and school culture. International Journal of Inclusive Education, 3(1), 53-61.

Creswell, J. W. (2014). Research design : qualitative, quantitative, and mixed methods approaches (4th ed..). Thousand Oaks: Thousand Oaks : SAGE Publications.

Crooks, V. a., \& Chouinard, V. (2006). An embodied geography of disablement: Chronically ill women's struggles for enabling places in spaces of health care and daily life. Health and Place, 12(3), 345-352.

Crooks, V. a., Chouinard, V., \& Wilton, R. D. (2008). Understanding, embracing, rejecting: Women's negotiations of disability constructions and categorizations after becoming chronically ill. Social Science and Medicine, 67(11), 1837-1846.

Crooks, V. a., Dorn, M. L., \& Wilton, R. D. (2008). Emerging scholarship in the geographies of disability. Health \& Place, 14(4), 883-888.

Curcic, S., Gabel, S., Zeitlin, V., Cribaro-Difatta, S., \& Glarner, C. (2011). Policy and challenges of building schools as inclusive communities. International Journal of Inclusive Education, 15(1), 117-133.

Dear, M., Wilton, R., Gaber, S. L., \& Takahashi, L. (1997). Seeing people differently: the sociospatial construction of disability. Environment and Planning D: Society and Space, 15(4), 455-480.

Department of Labour. (2011). New Land, New Life: Long-Term Settlement of Refugees in New Zealand (Preliminary Report). Quota Refugees Ten Years On. Wellington: Ministry of Business, Innovation and Employment. Retrieved from http://www.dol.govt.nz/publications/research/new-life-new-land/new-life-newland.pdf

Department of Labour. (2012). Quota refugees ten years on series. Quota Refugees Ten Years On: Findings from the Focus Groups " We'd like to live the life of New Zealanders. Wellington.

Detel, W. (2015). Social Constructivism. In International Encyclopedia of the Social \& Behavioral Sciences (2nd Edition, pp. 228-234). Elsevier Ltd.

Dias, K., \& Blecha, J. (2007). Feminism and social theory in geography: An introduction. Professional Geographer, 59(1), 1-9. 
Dyck, I. (2000). Disability, Geography and Ethics: Putting ethical research into practice: Issues of context. Philosophy \& Geography, 3(1), 80-87.

Ebersold, S., \& Evans, P. (2003). Disability in Higher Education. Paris: Paris :

Organisation for Economic Co-operation and Development.

Evans, I., Harvey, S., Buckley, L., \& Yan, E. (2009). Differentiating classroom climate concepts: Academic, management, and emotional environments. Kotuitui: New Zealand Journal of Social Sciences Online, 4(2), 131-146.

Freire, P. (1993). Pedagogy of the oppressed / Paulo Freire New York: New York: Continuum.

Gable, A. S. (2014). Disability theorising and real-world educational practice: a framework for understanding. Disability \& Society, 29(1), 86-100.

Garland-Thompson, R. (2004). Integrating disability, transforming feminist theory. In B. G. Smith \& B. Hutchinson (Eds.), Gendering Disability (pp. 73-106). New Brunswick, N.J: Rutgers University Press.

Gatens, M. (1996). Imaginary bodies : ethics, power, and corporeality London : London

Gatens, M. (1999). Feminist Theory and the Body: A Reader. In J. Price \& M. Shildrick (Eds.), (p. 487). Edinburgh, Scotland: Edinburgh, Scotland : Edinburgh University Press.

Gleeson 1964, B. (1999). Geographies of disability. New York; London: Routledge.

Goodley, D., \& Runswick Cole, K. (2014). Critical psychologies of disability: boundaries, borders and bodies in the lives of disabled children. Emotional and Behavioural Difficulties, 20(1), 51-63.

Grech, S., \& Soldatic, K. (2015). Disability and colonialism: (dis)encounters and anxious intersectionalities. Social Identities, 21(1), 1-5.

Hall, E., \& Kearns, R. (2001). Making space for the "intellectual" in geographies of disability. Health and Place, 7(3), 237-246.

Hansen, N., \& Philo, C. (2007). The normality of doing things differently: Bodies, spaces and disability geography. Tijdschrift Voor Economische En Sociale Geografie, 98(4), 493-506. 
Hassanein, E. E. A. (2015). Inclusion, Disability and Culture. Rotterdam:

SensePublishers.

Helliwell, E. (2014). Development and Disability: Engaging people with impairments in the Pacific. Victoria University of Wellington. Unpublished.

Higgins, N., MacArthur, J., \& Morton, M. (2013). Winding Back the Clock: The Retreat of New Zealand Inclusive Education Policy. The New Zealand Annual Review of Education, 17.

Hogansen, J. M., Powers, K., Geenen, S., Gil-Kashiwabara, E., \& Powers, L. (2008). Transition goals and experiences of females with disabilities: youth, parents, and professionals. Exceptional Children, 74(2), 215-234.

Holt, L. (2003). (Dis)abling children in primary school micro-spaces: Geographies of inclusion and exclusion. Health and Place, 9(2), 119-128.

Holt, L. (2010). Young people's embodied social capital and performing disability. Children's Geographies, 8(1), 25-37.

Holton, M., \& Riley, M. (2013). Student Geographies: Exploring the Diverse Geographies of Students and Higher Education. Geography Compass. Oxford, UK.

Humpage, L. (2009). A “culturally unsafe" space? The Somali experience of Christchurch secondary schools. New Zealand Geographer, 65(1), 73-82.

Immigration New Zealand. (2013). Refugee Settlement: New Zealand Resettlement Strategy. Wellington. Retrieved from http://www.immigration.govt.nz/NR/rdonlyres/1F4F5231-0974-430F-AE7ACD11CAE76227/0/RefugeeResettlementStrategy.pdf

Immigration New Zealand. (2015). The Refugee and Protection Unit Refugee Quota Branch (RQB) Statistics. Wellington. Retrieved from http://www.immigration.govt.nz/NR/rdonlyres/6D69584E-60E0-4D16-93D8F6C00BC384F1/0/RQBArrivalsStatPak.pdf

Immigration New Zealand. (2016). New Zealand Refugee Quota Programme. Retrieved March 23, 2016, from http://www.immigration.govt.nz/migrant/general/generalinformation/refugeeprotection/newzealandrefugeequotaprogramme.htm 
Imrie, R., \& Edwards, C. (2007). The Geographies of Disability: Reflections on the Development of a Sub-Discipline. Geography Compass, 1(3), 623-640.

Kearney, A. (2016). The right to education: What is happening for disabled students in New Zealand? Disability Studies Quarterly, 36(1), 1-16.

Kearney, A., \& Kane, R. (2006). Inclusive education policy in New Zealand: reality or ruse? International Journal of Inclusive Education, 10(2-3), 201-219.

Kenway, J., \& Fahey, J. (2011). Getting emotional about “brain mobility.” Emotion, Space and Society, 4(3), 187-194.

Kenway, J., \& Youdell, D. (2011). The emotional geographies of education: Beginning a conversation. Emotion, Space and Society, 4(3), 131-136.

Kirk, J., \& Cassity, E. (2007). Minimum standards for quality education for refugee youth: programs and practice. Youth Studies Australia, 26(1), 50-56.

Komardjaja, I. (2001). New cultural geographies of disability: Asian values and the accessibility ideal. Social \& Cultural Geography, 2(1), 77-86.

Landorf, H., \& Nevin, A. (2007). Inclusive global education: implications for social justice. Journal of Educational Administration, 45(6), 711-723.

Lefebvre, H. (1991). The production of space / Henri Lefebvre . Oxford, U.K.: Oxford, U.K. : Blackwell.

Locher, B., \& Prügl, E. (2001). Feminism and Constructivism: Worlds Apart or Sharing the Middle Ground? International Studies Quarterly, 45(1), 111-129.

Lockhart, A. (2015). New Zealand Refugee Resettlement Strategy. Wellington:

Ministry of Business, Innovation and Employment. Retrieved from https://www.google.co.nz/url?sa=t\&rct=j\&q=\&esrc=s\&source=web\&cd=3\&cad=rj a\&uact $=8 \&$ ved $=0$ ahUKEwiNqN7i--

TLAhUJxWMKHSR2BVIQFggsMAI\&url=http\%3A\%2F\%2Fwww.immigration.g ovt.nz\%2FNR\%2Frdonlyres\%2F7138E6CC-AF1E-4F10-BDB3-

A7C74ED270FE\%2F0\%2Fnzrrsandrewlockhartdayone.

Macartney, B., and Morton, M. (2013). Kinds of participation: Teacher and special education perceptions and practices of "inclusion" in early childhood and primary school settings. International Journal of Inclusive Education 17(8), 776-792. 
Marlowe, J., Bartley, A., \& Hibtit, A. (2014). The New Zealand Refugee Resettlement Strategy: implications for identity, acculturation and civic participation. Kōtuitui: New Zealand Journal of Social Sciences Online, 9(2), 60-69.

Marlowe, J., \& Elliott, S. (2014). Global trends and refugee settlement in New Zealand. Kōtuitui: New Zealand Journal of Social Sciences Online, 9(2), 43-49.

Massey, D. B. (2005). For space. London: SAGE.

Matthews, J. (2008). Schooling and settlement: refugee education in Australia. International Studies in Sociology of Education, 18(1), 31-45.

McBrien, J. L. (2014). I ōrea te tuātara ka patu ki waho: Competing Priorities in the New Zealand Refugee Resettlement Strategy. Wellington. Retrieved from http://www.fulbright.org.nz/wp-content/uploads/2014/08/axford2014_mcbrien.pdf

Michailakis, D., \& Reich, W. (2009). Dilemmas of inclusive education. Alter, 3(1), 24 Minister for Disability Issues. (2001). The New Zealand Disability Strategy.

Wellington. Retrieved from http://www.odi.govt.nz/publications/nzds/index.html Ministry of Education. (2014). English for Speakers of Other Languages: Refugee Handbook for Schools. Wellington. Retrieved from http://www.education.govt.nz/assets/Documents/School/Supportingstudents/ESOL/refugee-handbook-June2014.pdf

Ministry of Education. (2016). Inclusive Education. Retrieved November 12, 2016, from http://www.education.govt.nz/school/running-a-school/inclusive-education/

Ministry of Education. (2015). Specialist Service Standards for ORS | Education in New Zealand. Retrieved January 10, 2016, from http://www.education.govt.nz/school/student-support/specialeducation/ors/resources-for-students-in-ors/specialist-service-standards-for-ors/

Mitchell, D. (2012). Ecological model for working with children with complex needs and their families/whanau: A review of the literature carried out for the New Zealand Ministry of Education. Wellington. Retrieved from http://www.education.govt.nz/assets/Documents/Ministry/consultations/Residential -Special-Schools-consultation/JoinedUp.pdf

Mortensen, A., Latimer, S., \& Yusuf, I. (2014). Cultural case workers in child disability 
services: an evidence-based model of cultural responsiveness for refugee families. Kōtuitui: New Zealand Journal of Social Sciences Online, 9(2), 50-59.

Mthethwa-sommers, S., \& Kisiara, O. (2015). Listening to Students from Refugee Backgrounds : Lessons for Education Professionals. Perspectives on Urban Education, 12(1), 1-10.

Nairn, K., \& Higgins, J. (2011). The emotional geographies of neoliberal school reforms: Spaces of refuge and containment. Emotion, Space and Society, 4(3), $180-186$.

New Zealand Government (1989). Education Act 1989. Retrieved November 122016 from http://www.legislation.govt.nz/act/public/1989/0080/latest/DLM175959.html

New Zealand Government. (2014). UNHCR Resettlement Handbook: NEW ZEALAND. Retrieved from http://www.unhcr.org/cgibin/texis/vtx/home/opendocPDFViewer.html?docid=3c5e59d04\&query=medical/d isabled category

Noffke, S., \& Somekh, B. (2011). Action Research. In C. Lewin \& B. Somekh (Eds.), Theory and methods in social research (2nd ed.., pp. 94-101). London: London : SAGE.

O’Connor, R., \& Hooper, L. (2014). “ Then came reality ”: lived experiences of refugee youth in their first 12 months in New Zealand. Retrieved from http://reliefweb.int/sites/reliefweb.int/files/resources/RC\%20Refugee\%20Youth\%2 0Resettlement\%20Report\%20LR\%20v4_0.pdf

O'Rourke, D. (2011). Closing pathways: refugee-background students and tertiary education. Kotuitui: New Zealand Journal of Social Sciences Online, 6(1-2), 2636.

Orme, J. (1997). The Case for Research into Practice. In G. McKenzie, J. Powell, \& R. Usher (Eds.), Understanding Social Research: Perspectives on Methodology and Practice (pp. 112-123). London: Falmer Press.

Parliamentary Library, \& Parliament of Australia. (2011). Refugee resettlement to Australia: what are the facts? Background Note. Canberra. Retrieved from http://parlinfo.aph.gov.au/parlInfo/download/library/prspub/1276913/upload_binar y/1276913.pdf;fileType=application/pdf\#search=\%22background note 
(parliamentary library, australia)\%22

Pinson, H., \& Arnot, M. (2010). Local conceptualisations of the education of asylumseeking and refugee students: from hostile to holistic models. International Journal of Inclusive Education, 14(3), 247-267.

Refugee Council of Australia. (2015a). Barriers to Education for People Seeking Asylum and Refugees on Temporary Visas. Melbourne. Retrieved from http://www.refugeecouncil.org.au/wp-content/uploads/2014/08/1512Education.pdf

Refugee Council of Australia. (2015b). Barriers to Education for People Seeking Asylum and Refugees on Temporary Visas. Melbourne.

Rutherford, G. (2008). Different Ways of Knowing? Understanding Disabled Students' and Teacher Aides' School Experiences within a Context of Relational Social Justice. University of Otago, Dunedin.

Said, E. W. (1978). Orientalism New York: New York : Pantheon Books.

Scott, D. (1997). Qualitative Approaches to Data Collection and Analysis. In G. McKenzie, J. Powell, \& R. Usher (Eds.), Understanding Social Research: Perspectives on Methodology and Practice (pp. 155-172). London: Falmer Press.

Selvaraj, J. (2015). Inclusive education in New Zealand: policies, politics and contradictions. International Journal of Inclusive Education, 19(1), 86-101.

Sidhu, R. K., \& Taylor, S. (2007). Educational provision for refugee youth in Australia: left to chance? Journal of Sociology, 43(3), 283-300.

Singal, N. (2008). Working towards inclusion: Reflections from the classroom. Teaching and Teacher Education, 24(6), 1516-1529.

Šiška, J., \& Habib, A. (2012). Attitudes towards disability and inclusion in Bangladesh: from theory to practice. International Journal of Inclusive Education, 3116(May 2013), 1-13.

Slee, R. (2001). Social justice and the changing directions in educational research: the case of inclusive education. International Journal of Inclusive Education, 5(2-3), $167-177$.

Slee, R. (2006). Limits to and possibilities for educational reform. International Journal 
of Inclusive Education, 10(2-3), 109-119.

Slewa-younan, S., Mond, J. M., Bussion, E., Melkonian, M., Mohammad, Y., Dover, H., ... Jorm, A. F. (2015). Psychological trauma and help seeking behaviour amongst resettled Iraqi refugees in attending English tuition classes in Australia. International Journal of Mental Health Systems, 9(1), 1-6.

Snyder-Grant, Dana (2001). The hierarchy of disability. (Let Me Tell You...). Inside $M S, 19(3), 54$.

Söder, M. (2006). Disability as a social construct: the labelling approach revisited. European Journal of Special Needs Education, 4(2), 117-129.

Stanek, Ł. (2011). Henri Lefebvre on space: architecture, urban research, and the production of theory. Minneapolis: University of Minnesota Press.

Stewart, D., Law, M., Young, N. L., Forhan, M., Healy, H., Burke-Gaffney, J., \& Freeman, M. (2014). Complexities during transitions to adulthood for youth with disabilities: person-environment interactions. Disability and Rehabilitation, 36(23), 1998.

Stewart-Withers, R., Banks, G., McGregor, A., \& Meo-Sewabu, L. (2014). Qualitative Research. In R. Scheyvens (Ed.), Development fieldwork : a practical guide. (2nd edition, pp. 59-81). London SAGE Publications Ltd.

Sultana, F. (2007). Reflexivity, positionality and participatory ethics: Negotiating fieldwork dilemmas in international research. ACME, 6(3), 374-385.

Swan, J. (2002). Disabilities, Bodies, Voices. In S. L. Snyder, B. J. Brueggemann, \& R. Garland-Thomson (Eds.), Disability studies : enabling the humanities / edited by Sharon L. Snyder, Brenda Jo Brueggemann, and Rosemarie Garland-Thomson. (pp. 283-295). New York: Modern Language Association of America.

Taylor, S., \& Sidhu, R. K. (2012). Supporting refugee students in schools: what constitutes inclusive education? International Journal of Inclusive Education, 16(1), 39-56.

Thiem, C. H. (2009). Thinking through education: the geographies of contemporary educational restructuring. Progress in Human Geography, 33(2), 154-173.

Thomas, C. (2004). How is disability understood? An examination of sociological 
approaches. Disability \& Society, 19(6), 569-583.

Thompson, C. M. (2015). Situated knowledge, feminist and science and technology studies perspectives. International Encyclopedia of the Social and Behavioral Sciences (Second Edi, Vol. 22). Elsevier.

Titchen, A., \& Hobson, D. (2011). Understanding Phenomenology through Reverse Perspectives. In C. Lewin \& Some (Eds.), Theory and methods in social research (2nd edition, pp. 121-130). London: SAGE Publications.

United Nations. (2006). Convention on the rights of persons with disabilities. Retrieved from http://www.un.org/disabilities/

United Nations High Commission for Refugees. (1957). the Convention Relating To the Status of Refugees. International and Comparative Law Quarterly, 6(3), 533-535.

United Nations High Commission for Refugees. (2015). UNHCR - Asylum-Seekers. Retrieved from http://www.unhcr.org/pages/49c3646c137.html

Waters, J. L. (2012). Geographies of International Education: Mobilities and the Reproduction of Social (Dis)advantage. Geography Compass. Oxford, UK.

West, S. (2004). School's in for Australia's refugee students. Principal Matters, (61), $30-32$.

Worth, N. (2008). The significance of the personal within disability geography. Area, 40(3), 306-314.

Worth, N. (2013). Making friends and fitting in: a social-relational understanding of disability at school. Social \& Cultural Geography, 14(1), 103-123.

Ypinazar, V., \& Pagliano, P. (2004). Seeking inclusive education: disrupting boundaries of "special" and "regular" education. International Journal of Inclusive Education, $8(4), 423-442$.

Zembylas, M. (2011). Investigating the emotional geographies of exclusion at a multicultural school. Emotion, Space and Society, 4(3), 151-159.

Zembylas, M. (2013). Mobilizing "implicit activisms" in schools through practices of critical emotional reflexivity. Teaching Education, 24(1), 84-96. 


\section{Appendix One: Interview Information Sheet}

Participant Information Sheet

Research Project Title: Inclusive Education Spaces for Refugee Background People with Disabilities

\section{Researcher: Lucy Croft, School of Geography, Environment and Earth Sciences, Victoria University of Wellington}

As part of the completion of my Masters of Science in Human Geography, this study is designed to investigate inclusive education spaces for refugee background people with disabilities, and assess improvements which need to be made. Victoria University requires, and has granted, approval from the School's Human Ethics Committee [xxx No.]

I am inviting service providers to participate in this research. Participants will be asked to take part in a hour long (approximately) interview. Permission will be asked to record the interview, and a transcript of the interview will be sent to participants for checking.

Participation is voluntary, and you will not be identified personally in any written report produced as a result of this research, however your organisation may be identified. This includes possible publication in academic conferences and journals. All material collected will be kept confidential, and will be viewed only by myself and my supervisors, Drs Sara Kindon and Bronwyn Wood. The thesis will be submitted for 
marking to the School of Geography, Environment and Earth Sciences, and subsequently deposited in the University Library. Should any participant wish to withdraw from the project, they may do so until three months after the scheduled interview, and the data collected up to that point will be destroyed. All data collected from participants will be destroyed within three years after the completion of the project.

If you have any questions or would like to receive further information about the project, please contact me at lucy.croft@vuw.ac.nz or telephone [details provided] or you may contact my supervisor Sara Kindon at sara.kindon@vuw.ac.nz or telephone (04) 463-6194. If you have any ethical concerns about the research, please contact $\mathrm{Dr}$ Susan Corbett (Chair of the Human Ethics Committee, Victoria University of Wellington) at susan.corbettvuw.ac.nz or phone (04) 4635480

Lucy Croft 


\section{Appendix Two: Interview Consent}

CONSENT TO PARTICIPATION IN RESEARCH

Title of project: Inclusive Education Spaces for Refugee Background People with

\section{Disabilities}

I have been given, and have understood, an explanation of this research project. I have had an opportunity to ask questions, and have them answered to my satisfaction. I understand that I may withdraw myself, or any information directly related to me, from this project before data collection and analysis is complete, without having to give reasons.

I understand that any information collected will be kept confidential to the researcher. My responses will be anonymous.

Final presentation of the data may be published in journals and/or conferences, but research is primarily directed towards the completion of Lucy Croft's Master's thesis.

I agree to take part in this research on inclusive education spaces for refugee background people with disabilities.

I would like to receive a summary of findings at the end of the research (please provide email)

Signed:

Date:

Name of Participant:

(Please print clearly)

Email address: 


\section{Appendix Three: Interview Questions}

Interview Guidelines

\section{Research Project Title: Inclusive Education Spaces for Refugee Background People with Disabilities}

\section{Researcher: Lucy Croft, School of Geography, Environment and Earth Sciences, Victoria University of Wellington}

Consent forms and information sheets will be provided to the interview participants. Confidentiality will be maintained between all participators in the interview, and a respectful, inclusive atmosphere will be present at the interview.

Questions for inclusive education service providers:

1. What does your role entail?

2. How much of your role involves working with refugee background people with disabilities?

3. How would you define disability?

4. How would you define inclusive education?

5. How would you define inclusion?

6. How would you describe inclusive education for refugee background people?

7. What are key aspects needed for inclusive education?

8. Does your organisation practice inclusive principles? Why?

9. How do you think inclusive education could be better practiced in your organisation?

10. What barriers do refugee background people with disabilities face in inclusive education?

11. How do you think your organisation mitigates these barriers?

12. Does your organisation communicate with other organisations related to refugee background people?

13. Could you describe a positive inclusive education space in your workplace?

14. Could you describe a negative inclusive education space in your workplace? 
15. What has been a positive experience that you have had working with refugee background people with disabilities? Why?

16. What has been a negative experience that you have had working with refugee background people with disabilities? Why?

17. Could you share an image of what an ideal inclusive education space would be for you?

18. How do you think inclusive education for refugee background people with disabilities could be improved in New Zealand?

19. How do you think arrangements for refugee background people with disabilities could be improved?

\section{Questions for government workers:}

1. What does your role entail?

2. How much of your role involves working with refugee background people with disabilities?

3. How would you define disability?

4. How would you define inclusive education?

5. How would you define inclusion?

6. How would you describe inclusive education for refugee background people?

7. What are key aspects needed for inclusive education?

8. Does your organisation practice inclusive principles? Why?

9. How do you think inclusive education could be better practiced in New Zealand?

10. How could inclusive education be incorporated into New Zealand policy?

11. Does your government organisation communicate with other

12. How would you describe a positive inclusive education space?

13. How would you describe a negative inclusive education space?

14. What has been a positive experience that you have had working with refugee background people with disabilities (either in policy or practice)? Why?

15. What has been a negative experience that you have had working with refugee background people with disabilities (either in policy or practice)? Why?

16. What are some barriers to improving inclusive education for refugee background people with disabilities?

17. Could you share an image of what an ideal inclusive education space would be for you?

18. How do you think inclusive education for refugee background people with disabilities could be improved in New Zealand? 
19. How do you think arrangements for refugee background people with disabilities could be improved?

20. How could the government improve policies for refugee background people?

\section{Questions for refugee background people with disabilities:}

1. Could you please state your education experiences before arriving in New Zealand?

2. Could you please state your education experiences while in New Zealand?

3. What has been a positive experience of education in New Zealand?

4. What has been a negative experience of education in New Zealand?

5. Could you describe the place where you felt most comfortable learning in? What did it look like?

6. What has helped you to learn in New Zealand?

7. What hasn't helped you to learn in New Zealand?

8. Who has helped you in your experiences of education in New Zealand?

9. Who hasn't helped you in your experiences of education in New Zealand?

10. What improvements could be made to education in New Zealand?

11. How has your disability affected your educational experience?

12. What would your ideal educational space look like? 